\title{
Scaling Theory of the Integer Quantum Hall Effect
}

\author{
Bodo Huckestein* \\ Max-Planck-Institut für Kernphysik, 69029 Heidelberg, Germany
}

(February 1, 2008)

\begin{abstract}
The scaling theory of the transitions between plateaus of the Hall conductivity in the integer Quantum Hall effect is reviewed. In the model of twodimensional noninteracting electrons in strong magnetic fields the transitions are disorder-induced localization-delocalization transitions. While experimental and analytical approaches are surveyed, the main emphasis is on numerical studies, which successfully describe the experiments. The theoretical models for disordered systems are described in detail. An overview of the finitesize scaling theory and its relation to Anderson localization is given. The field-theoretical approach to the localization problem is outlined. Numerical methods for the calculation of scaling quantities, in particular the localization length, are detailed. The properties of local observables at the localizationdelocalization transition are discussed in terms of multifractal measures. Finally, the results of extensive numerical investigations are compared with experimental findings.
\end{abstract}

\section{Contents}

I Introduction

*Present address: Institut für Theoretische Physik, Universität zu Köln, 50937 Köln, Germany 


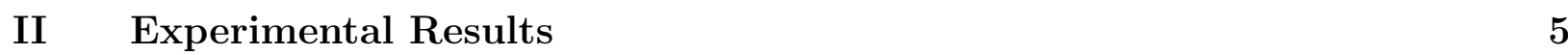

A Temperature-Dependent Scaling . . . . . . . . . . . . . . . 6

B Size-Dependent Scaling . . . . . . . . . . . . . . . . . . . . . . . 9 9

C Frequency-Dependent Scaling . . . . . . . . . . . . . . . . . . . . . 9

\begin{tabular}{ll}
\hline III $\quad$ Models of Disorder & 10
\end{tabular}

A Real-Space Models . . . . . . . . . . . . . . . . . . . . 12

B Landau-Space Models . . . . . . . . . . . . . . . . . . . . . . 14

C Lattice Models . . . . . . . . . . . . . . . . . . . . . . . . . . 19

D Semiclassical Approximation and Network Model . . . . . . . . . . . . . . 21

\begin{tabular}{llr}
\hline IV & Finite-Size Scaling & 28
\end{tabular}

A Single-Parameter Scaling . . . . . . . . . . . . . . . . . . 28

B Corrections to Scaling . . . . . . . . . . . . . . . . . . 33

C Dynamic Scaling . . . . . . . . . . . . . . . . . . . 34

\begin{tabular}{|lll}
\hline$\quad$ Scaling Behavior and Field Theory & 34 \\
\hline
\end{tabular}

A Anderson Localization . . . . . . . . . . . . . . . . . . . . 35

B Nonlinear Sigma Model and Topological Term . . . . . . . . . . . . . . . . 38

\begin{tabular}{|lr}
\hline VI $\quad$ Localization Length & 40 \\
\hline
\end{tabular}

A Recursive Green Function Method . . . . . . . . . . . . . . . . . . . . . . 41

B Transfer-Matrix Method . . . . . . . . . . . . . . . . . . . . . 45

\begin{tabular}{|ll}
\hline VII Other Scaling Quantities & 46
\end{tabular}

A Thouless Number . . . . . . . . . . . . . . . . . 46

B Chern Number . . . . . . . . . . . . . . . . . . . 47

\begin{tabular}{|lr}
\hline VIII Numerical Calculations & 48
\end{tabular}

A $\quad$ Lowest Landau Level . . . . . . . . . . . . . . . . . . . . . . . . . . . . . . 49

B Higher Landau Levels . . . . . . . . . . . . . . . . . . . . . . . 54 
C Network mode . . . . . . . . . . . . . . . . . . . . . . . . . . . . . 59

D Effects of Landau Level Coupling and Spin-Orbit Interaction . . . . . . . 60

E Presence of a Periodic Potential . . . . . . . . . . . . . . . . . . . 62

\begin{tabular}{lll}
\hline IX & Multifractal Analysis & 63
\end{tabular}

A Generalized Dimensions and Singularity Strengths . . . . . . . . . . . 64

B Universality of Multifractal Spectra . . . . . . . . . . . . . . . . . . . . 69

C Multifractality and Scaling . . . . . . . . . . . . . . . . . . 70

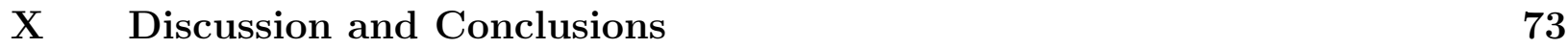

\begin{tabular}{ll}
\hline APPENDIXES & 77
\end{tabular}

\section{INTRODUCTION}

As announced in a paper by von Klitzing, Dorda, and Pepper (1980), Klaus von Klitzing discovered that under certain experimental conditions the Hall conductivity $\sigma_{x y}$ of a quasi-two-dimensional electron gas is quantized to a high precision in integer multiples of $e^{2} / h$ while at the same time the longitudinal conductivity $\sigma_{x x}$ vanishes. A remarkable aspect of this integer quantum Hall effect (QHE) is that the quantization exists over a finite range of physical parameters, like the magnetic field $B$ or the carrier concentration $n$. This observation cannot be explained by the semiclassical Drude theory of conductivity. If the longitudinal conductivity $\sigma_{x x}$ vanishes the Drude result for the Hall conductivity is given by $\sigma_{x y}=\nu e^{2} / h$, where $\nu=n h / e B=n 2 \pi l_{c}^{2}$ is the filling factor. Only if $\nu$ is an integer, i.e., if the Fermi level lies exactly between two Landau levels, is the Hall conductivity quantized. Laughlin (1981), Aoki and Ando (1981), and Halperin (1982) showed that the Hall conductivity takes on its quantized value $i e^{2} / h$ over a finite range in filling factor around $\nu=i$ if in that range the states at the Fermi energy are localized. By the same argument, when the Hall conductivity is quantized there are states below the Fermi energy that are 
not localized. The width of the quantized plateaus then depends on the ratio of localized vs. extended states.

The concept of localized states in disordered systems was developed by Anderson (1958). He showed that if a quantum-mechanical system is sufficiently disordered, states have a finite probability to return to a given site in the long-time limit. This absence of diffusion implies that these states are localized in a finite region of space. The transmission probability decays exponentially on a length scale, which is called the localization length. For localized states the static conductivity vanishes at zero temperature. On the other hand, if the disorder is weak enough extended states might exist that do not decay exponentially and fill the whole system. Their contribution to the conductivity is finite even at zero temperature. The energy that separates extended from localized states is called the mobility edge. At the mobility edge the character of eigenstates is different from both extended and localized states and we will call these states critical states.

The observation that an explanation of the QHE involves both extended and localized states was the more unanticipated as the scaling theory of localization (Wegner, 1976; Abrahams et al., 1979; Wegner, 1979) predicted the absence of extended states in two-dimensional systems. It is the presence of a strong magnetic field that leads to the emergence of nonlocalized states in two dimensions (Ono, 1982b). Chalker (1987) showed that these states exist only at a single energy in the limit that scattering between Landau levels can be neglected. At zero temperature the Hall conductivity is thus expected to exhibit sharp steps whenever the Fermi energy passes the critical energy. The longitudinal conductivity vanishes for all energies except at the critical energy. The quantum Hall plateau transition is thus a special case of a metal-insulator transition. The insulating phases correspond to the plateau regions, in which $\sigma_{x x}$ vanishes and $\sigma_{x y}$ is quantized. These insulating phases are separated by mobility edges with a finite $\sigma_{x x}$. Truly metallic phases are absent in the quantum Hall system. The states at the mobility edge show critical fluctuations (Wegner, 1980; Aoki, 1983; Aoki, 1986).

The description of this localization-delocalization transition in terms of a zero- 
temperature phase transition is the topic of this review. We will focus on the theory of noninteracting electrons. Thus we do not consider the fractional QHE (Tsui et al., 1982), where the quantization of the Hall conductivity in rational fractions of $e^{2} / h$ is due to electronelectron interactions. One notes, however, that experimentally the fractional quantum Hall transitions show a remarkable similarity to the integer transitions (see Sec. ПI). In the absence of an analytical theory that is capable of providing quantitative results we will mostly rely on numerical simulations.

The review is organized as follows: In Sec. II we shortly survey the experimental investigations of the quantum Hall transitions. Section III discusses the different models of disorder used in theoretical calculations. Section $\mathbb{I V}$ provides an overview of the finite-size scaling theory. Section $\mathrm{V}$ outlines the description of the Anderson transition in terms of a field theory and its extension to the quantum Hall system. Sections VI and VII explain which physical quantities are used in numerical finite-size scaling studies and how they are computed. In Sec. VIII the results of numerical calculations are presented and analyzed in terms of the finite-size scaling theory. In Sec. [X] we characterize the critical states using a multifractal analysis. In Sec. \ we compare the experimental and theoretical results presented in the previous sections and discuss their implications for an understanding of the quantum Hall transition. Except where noted otherwise we try to provide enough detail to allow the reader to use this review as a starting point for own investigations of the subject.

A comprehensive introduction to the QHE is provided in the book edited by Prange and Girvin (1987). For reviews about disordered electronic systems and localization see Thouless (1974), Lee and Ramakrishnan (1985), and Kramer and MacKinnon (1993).

\section{EXPERIMENTAL RESULTS}

In this section we want to review experimental investigations of the transition between plateaus in the Hall conductivity. We will focus on those results that are uniquely related to these phase transitions. In later sections we will show how a simple scaling theory for 
the integer quantum Hall transitions accounts for most of the observed features. We will therefore postpone the discussion of the experimental results until we are familiar with the necessary theoretical framework (Sec. X]).

The sections $\llbracket \mathrm{A}$ to $\amalg \mathrm{C}$ are ordered roughly chronologically. We will start with exper-

iments on the temperature dependence of the transition. Although these experiments give the most indirect information about the scaling behavior they were the first to be performed and instrumental in developing the idea that the plateau transitions are continuous phase transitions. Much more direct contact with the concept of finite-size scaling is made in experiments with varying sample sizes. Finally, the dynamic aspects of the transition are elucidated in high-frequency experiments.

\section{A. Temperature-Dependent Scaling}

Soon after the discovery of the QHE (von Klitzing et al., 1980) it became apparent that the quantized plateaus can become extremely broad and the transition between them extremely sharp as the temperature was lowered (Kawaji and Wakabayashi, 1981; Paalanen et al., 1982). At the same time the longitudinal conductivity exhibits a series of sharp spikes at the position of the plateau transitions. Since the Fermi energy is in a region of localized states when the Hall conductivity is quantized and the longitudinal conductivity vanishes, it can be concluded from the wide plateaus that most of the electronic states are localized at low temperatures. Paalanen, Tsui, and Gossard (1982) estimated that in an AlGaAs/GaAs heterostructure at $50 \mathrm{mK} 95 \%$ of the states in each Landau level are localized.

The temperature dependences of the longitudinal conductivity $\sigma_{x x}$ and the Hall conductivity $\sigma_{x y}$ of a Si-MOSFET are shown in Fig. 1. Even in the absence of localization the temperature dependence of the Fermi-Dirac distribution leads to a temperature dependence of the conductivity,

$$
\sigma_{\mu \nu}(T)=\int d E\left(-\frac{\partial f(T)}{\partial E}\right) \sigma_{\mu \nu}(T=0) .
$$


If $\sigma_{x x}(T=0)$ is finite only in an interval of width $\Delta$ near the center of each Landau band, Eq. (2.1) predicts an increase of $\sigma_{x x}$ in the band center with decreasing temperature as long as $k_{B} T \gtrsim \Delta$. This increase is seen in the peaks of Fig. 1.

When plotting $\sigma_{x x}$ versus $\sigma_{x y}$ as a function of temperature and filling factor striking features appear. The data for a Si-MOSFET for temperatures between $0.35 \mathrm{~K}$ and about $1 \mathrm{~K}$ in Fig. 2 lie on a single curve indicating that $\sigma_{x x}$ and $\sigma_{x y}$ are not independent but dependent on a single parameter. At higher temperatures the effect of the Fermi distribution is still visible in the increase of $\sigma_{x x}$. By contrast, the conductivities seem to be independent and follow a two-parameter scaling flow in the temperature driven flow diagram for an InGaAsInP heterostructure in Fig. 3. In both figures a flow towards $\left(\sigma_{x x}, \sigma_{x y}\right)=(0$, integer $) e^{2} / h$, corresponding to the widening plateaus, is obvious. Kravchenko and Pudalov (1989) and Kravchenko et al. (1990) studied Si-MOSFET and AlGaAs/GaAs heterostructures and observed both types of behavior depending on the filling factor. A single curve was also obtained by McEuen et al. (1990) in a study of the non-local transport properties in highmobility GaAs-AlGaAs samples.

It is experimentally extremely difficult to obtain reliable flow-diagrams close to the transitions at half-integer Hall conductivity. As the temperature approaches zero the transition regions become very narrow and inhomogeneities in the electron density will let the transition happen at slightly different values of the magnetic field in different parts of the sample. Since $\sigma_{x x}$ and $\sigma_{x y}$ are measured on different parts of the sample this will lead to irregular behavior near the transition. Wei, Tsui, Paalanen, and Pruisken (1988) avoided this problem by studying the critical behavior of each component of the resistivity tensor separately. Fig. 4 shows the transport coefficients $\rho_{x y}, \rho_{x x}$, and the derivative $d \rho_{x y} / d B$ for an InGaAs-InP heterostructure. Note the similarity between $\rho_{x x}$ and $d \rho_{x y} / d B$. However, while the maximum values of the former decrease, the maximum values of the latter increase with decreasing temperature. In Fig. 5 the temperature dependence of the maximum $\left(d \rho_{x y} / d B\right)^{\max }$ and the inverse width $(\Delta B)^{-1}$ of the $\rho_{x x}$ peaks is plotted for several Landau levels. Over the range of temperatures from $0.1 \mathrm{~K}$ to $4.2 \mathrm{~K}$ they show power-law behavior $\left(d \rho_{x y} / d B\right)^{\max } \propto T^{-\kappa}$ and 
$\Delta B \propto T^{\kappa}$ with $\kappa=0.42 \pm 0.04$. Further measurements of higher-order derivatives showed that the extrema of $d^{2} \rho_{x x} / d B^{2}$ and $d^{3} \rho_{x y} / d B^{3}$ diverge like $T^{-2 \kappa}$ and $T^{-3 \kappa}$, respectively (Wei et al., 1990). In experiments on low-mobility AlGaAs/GaAs samples the same exponent was observed but the characteristic temperature below which this scaling was observed was much lower $(200 \mathrm{mK})$ than in the InGaAs/InP samples (4.2 K) (Wei et al., 1992). Huckestein et al. (1991) studied the temperature dependence of the plateau width in AlGaAs/GaAs heterostructures and found agreement with $\kappa=0.42$ for temperatures below $4.2 \mathrm{~K}$. For Landau levels where the spin splitting was not resolved $\left(d \rho_{x y} / d B\right)^{\max }$ and $(\Delta B)^{-1}$ diverge like $T^{-\kappa / 2}$ (Wei et al., 1990; Hwang et al., 1993). The same exponent $\kappa$ was measured in the fractional QHE regime of high-mobility AlGaAs/GaAs heterostructures for the scaling between filling factors $2 / 5$ and $1 / 3$ (Engel et al., 1990).

The universality of the exponent $\kappa$ was questioned by Wakabayashi et al. (1989; 1990) and by Koch et al. (1991a). Wakabayashi et al. studied two of the valley- and spin-split subbands of Landau levels $n=0$ and $n=1$ in a Si-MOSFET. For the rather limited temperature range between $0.35 \mathrm{~K}$ and $1 \mathrm{~K}$ they observe $\kappa=0.29 \pm 0.10$ and $\kappa=0.16 \pm 0.02$ for subbands $(0 \downarrow-)$ and $(1 \uparrow-)$, respectively. Below $0.2 \mathrm{~K}$ the temperature dependence saturates, presumably due to Joule heating (Wakabayashi et al., 1990). Koch et al. found a dependence of $\kappa$ on the mobility of AlGaAs/GaAs heterostructures deliberately doped to decrease the mobility. They observed power-law scaling with temperature over the range 40 $\mathrm{mK}$ to $1.1 \mathrm{~K}$. For spin-split Landau levels the measured values for $\kappa$ increased from 0.28 to 0.81 with decreasing mobility. If the spin splitting is not resolved, $\kappa$ is considerably smaller but not by a universal factor of two as found by Wei et al. (Koch et al., 1991a).

In the context of the finite-size scaling theory (see Sec. IV) the power law behavior of the transport coefficients reflects the single-parameter scaling of the conductivity tensor (Pruisken, 1988)

$$
\sigma_{\mu \nu}(B)=\frac{e^{2}}{h} S_{\mu \nu}\left(L_{\mathrm{eff}}^{1 / \nu}\left(B-B^{*}\right)\right),
$$

where $B^{*}$ is the critical magnetic field of the plateau transition, $L_{\text {eff }}$ is the effective system 
size, and $\nu$ is the critical exponent of the localization length [cf. Eq. (4.3)]. The strongest support for this assertion comes from the peculiar temperature dependence of the magnetic field derivatives of the conductivity tensor. At $T=0, L_{\text {eff }}$ is equal to the system size. At finite temperatures the effective system size is given by the phase coherence length $L_{\Phi}$ (Thouless, 1977). If $L_{\Phi}$ diverges as $T^{-p / 2}$ as the temperature approaches zero then $L_{\mathrm{eff}}^{1 / \nu} \propto T^{-\kappa}$ with $\kappa=p / 2 \nu$. With Eq. (2.2) the $n$-th derivative of the conductivity tensor at the critical point is

$$
\frac{d^{n} \sigma_{\mu \nu}\left(B^{*}\right)}{d B^{n}} \propto L_{\mathrm{eff}}^{n / \nu} \propto T^{-n \kappa}
$$

unless it vanishes due to symmetry.

\section{B. Size-Dependent Scaling}

Koch et al. (1991b) measured the exponent $\nu$ directly by studying samples of the same shape but different sizes. For sufficiently small samples $\left(d \rho_{x y} / d B\right)^{\max }$ and $\Delta B$ saturate at low temperatures (see Fig. 6). The saturation temperature decreases with increasing system size. This is interpreted as the temperature where the phase coherence length $L_{\Phi}$ becomes comparable to the system size and the temperature-dependent scaling at higher temperature crosses over to size-dependent scaling. The saturation value of $\Delta B$ at low temperatures is then given by the condition $L / \xi(\Delta B) \approx 1$, i.e., $\Delta B \propto L^{-1 / \nu}$. By fitting their data to this relation Koch et al. obtain $\nu=2.3 \pm 0.1$ for the three lowest Landau levels. From the measured values of $\kappa$ they deduce that $p$ is not universal and varies between 2.7 and 3.4. For a Landau level in which the spin splitting was not resolved $\nu=6.5 \pm 0.6$ was measured while the value of $p$ was comparable to its value in other Landau levels.

\section{Frequency-Dependent Scaling}

The dynamical conductivity $\sigma_{x x}(f)$ of a low-mobility AlGaAs/GaAs heterostructure was investigated by Engel et al. (1993) by measuring the attenuation of a coplanar transmis- 
sion line on the sample surface. The losses in the transmission line are due to the finite conductivity of the two-dimensional electron gas below the surface. In contrast to previous measurements using crossed rectangular waveguides (Kuchar et al., 1986; Huckestein et al., 1991) that were limited to a single frequency $f=35 \mathrm{GHz}$ Engel et al. were able to sweep the frequency from 0.2 to $14 \mathrm{GHz}$. As can be seen from Fig. 7 the effects of raising the temperature and increasing the frequency are quite similar. With decreasing frequency the width $\Delta B$ of the peaks in $\operatorname{Re}\left(\sigma_{x x}\right)$ decreases and saturates below a temperature-dependent frequency. Above this frequency $\Delta B$ scales like

$$
\Delta B \propto f^{\gamma}
$$

with $\gamma=0.41 \pm 0.04$ for spin-split Landau levels and $\gamma=0.21$ for non-split Landau levels. The saturation temperature corresponds to $h f \approx k_{B} T$ in agreement with the results of Huckestein et al. (1991).

Similar to Eq. (2.2) a dynamical scaling ansatz for $\sigma_{x x}(L, f)$ is

$$
\sigma_{x x}(L, f)=\frac{e^{2}}{h} S_{x x}\left(L / \xi(B), f \tau_{0}(B)\right),
$$

where $\tau_{0}(B) \propto \xi(B)^{z} \propto\left|B-B^{*}\right|^{-\nu z}$ is the relaxation time of the system that diverges as the critical point is approached. For sufficiently high frequencies $f \tau_{0}(B)$ dominates the scaling behavior of $S_{x x}$ and $\Delta B$ scales like $f^{1 / \nu z}$. Equation (2.4) thus implies $\gamma=1 / \nu z$, and using the result $\nu=2.3$ from the size-dependent scaling experiments, $z=1$ is obtained (Engel et al., 1993).

\section{MODELS OF DISORDER}

Before we can attempt to understand the experiments we have to define our system in theoretical terms. We will make several simplifying assumptions: (1) The electron system is strictly two-dimensional. (2) The interactions between the electrons can be neglected. (3) The spin degrees of freedom can be neglected. (4) Boundary effects, like edge states, are not 
essential in understanding the critical behavior of the system. The electron gas in real devices is only quasi-two-dimensional, in the sense that the motion perpendicular to the plane of the electron gas is quantized into electrical subbands. If only the lowest electrical subband is occupied the electrons have only two spatial degrees of freedom left and we may treat them as two-dimensional, justifying our assumption (1). Assumption (2) lacks an a priori justification. The treatment of non-interacting electrons will turn out to be quite successful in explaining the experimental static-scaling results. While this might serve as an a posteriori justification of our approach a theoretical assessment of the relevance of electron-electron interactions is still missing. For an understanding of the observed dynamical scaling it seems to be essential to go beyond the non-interacting electron approximation. Assumption (3) seems justified since most experiments deal with completely spin-split Landau level and we neglect interactions between the electrons. For non-spin-split levels spin-orbit scattering might be important and one would have to go beyond the spinless-electron approximation. The last assumption (4) treats the phase transition in the spirit of standard scaling theory as a bulk critical phenomenon. We will make use of this assumption by choosing boundary conditions most suitable to the calculation at hand.

We will thus describe our system by a single-particle Hamiltonian

$$
H=H_{0}+V(\mathbf{r})
$$

where

$$
H_{0}=\frac{1}{2 m}(\mathbf{p}-e \mathbf{A})^{2}
$$

is the kinetic energy of the electron and $V(\mathbf{r})$ is the disorder potential. In the absence of disorder, $V(\mathbf{r})=0$, the spectrum of $H$ for an infinite system is a set equidistant levels,

$$
E_{n}=(n+1 / 2) \hbar \omega_{c}
$$

separated by the cyclotron energy $\hbar \omega_{c}=\hbar e B / m$. Each Landau level $n$ is infinitely degenerate with a degeneracy of 


$$
n_{B}=1 / 2 \pi l_{c}^{2}
$$

states per unit area. The length $l_{c}=(\hbar / e B)^{1 / 2}$ is called the magnetic length and is the classical cyclotron radius in the lowest Landau level $n=0$.

In order to fully define the model system we will now describe different models of the disorder potential typically used in calculations.

\section{A. Real-Space Models}

The statistical properties of a disorder potential are completely determined by the joint

probability distribution $P[V]$ of the disorder potential $V(\mathbf{r})$ for all coordinates $\mathbf{r}$. Starting point for most analytical calculations is the white-noise potential distribution

$$
P[V]=\mathcal{N} \exp \left\{-\frac{1}{2 V_{0}^{2}} \int d^{2} r[V(\mathbf{r})]^{2}\right\},
$$

where $\mathcal{N}$ normalizes the distribution. With this distribution the potential at different coordinates is uncorrelated,

$$
\overline{V(\mathbf{r}) V\left(\mathbf{r}^{\prime}\right)}=V_{0}^{2} \delta\left(\mathbf{r}-\mathbf{r}^{\prime}\right)
$$

and has a Gaussian distribution at each point in space. The overbar denotes the average with respect to the distribution $P[V]$ of the disorder potential. This potential distribution is well suited for analytic calculations since it allows one to perform the average over disorder, due to the Gaussian dependence on the potential. For the white-noise distribution Wegner (1983) showed that the density of states in the lowest Landau level is given by

$$
\rho(E)=\frac{\sqrt{2}}{\pi^{2} l_{c} V_{0}} \frac{\exp \left(\nu^{2}\right)}{1+\left(2 \pi^{1 / 2} \int_{0}^{\nu} d x \exp \left(x^{2}\right)\right)^{2}},
$$

where

$$
\nu=\frac{\sqrt{2 \pi} l_{c}}{V_{0}}\left(E-\frac{1}{2} \hbar \omega\right) .
$$


If the integrand in the exponent of Eq. (3.5) contains a more complicated function of $V(\mathbf{r})$, but still depends only on the potential at one point in space, the potential is still $\delta$-correlated, but higher-order cumulants of the potential at the same site have non-zero coefficients. Brézin, Gross, and Itzykson (1984) generalized Wegner's result (3.7) to this more general class of potentials.

For numerical calculations it is not practical to specify a random value for the potential at every point in space. Instead one uses an approximation to the white-noise distribution Eq. (3.5), the $\delta$-scatterers potential,

$$
V(\mathbf{r})=\sum_{i=1}^{N} V_{i} \delta\left(\mathbf{r}-\mathbf{r}_{i}\right),
$$

where the sites $\mathbf{r}_{i}$ of the scatterers are randomly chosen and there is an equal number of attractive $V_{i}=-V$ and repulsive scatterers $V_{i}=+V$. This potential has the correlation function (3.6) and approaches the white-noise potential (3.5) with $V_{0}^{2}=n_{i} V^{2}$ in the limit that the density of scattering sites $n_{i}$ becomes infinite. Due to the symmetry of the potential the density of states is symmetric with respect to the center of the Landau level. The self-consistent Born approximation (SCBA) for the density of states of this potential is a semi-circle,

$$
\rho(E)=\frac{1}{2 \pi l_{c}^{2}} \frac{2}{\pi \Gamma}\left[1-\left(\frac{E-E_{n}}{\Gamma}\right)^{2}\right]^{1 / 2},
$$

with the bandwidth $\Gamma=\left(4 n_{i} V^{2} / 2 \pi l_{c}^{2}\right)^{1 / 2}=2 V_{0} / \sqrt{2 \pi} l_{c}$ (Ando and Uemura, 1974). Since this is also the energy scale in Eq. (3.7) we will frequently use $\Gamma$ as a measure of the width of the Landau level.

In numerical calculations care has to be taken that the concentration $c_{i}=2 \pi l_{c}^{2} n_{i}$ of scatterers is large enough in order to avoid a singularity in the density of states due to wavefunctions avoiding the scatterers (Ando and Aoki, 1985). To see the origin of this effect consider a square of side $L$ containing an integral number of flux quanta $N_{S}=L^{2} / 2 \pi l_{c}^{2}$. From Eq. (3.4) we see that $N_{S}$ is also the number of degenerate eigenstates of the system in the absence of disorder. We can form a superposition of these $N_{S}$ states that satisfies $N_{S}$ 
constraints. Thus if the number $N_{i}=n_{i} l_{c}^{2}$ of $\delta$-scatterers is less than $N_{S}$, the wavefunction can be made to vanish at the site of each scatterer and hence the energy of this state will not be influenced by the disorder potential. This leads to a divergence of the density of states at $E=\hbar \omega_{c} / 2$ even in the presence of disorder. In terms of the concentration $c_{i}$, this divergence will occur for $c_{i}<1$. Ando and Aoki state that for $c_{i}=40$ a good approximation to the white-noise limit is achieved (Ando and Aoki, 1985).

Disorder potentials with a finite correlation length can be generated by replacing the $\delta$-functions in Eq. (3.8) by functions with a finite range. For Gaussian scatterers,

$$
\begin{aligned}
& V(\mathbf{r})=\sum_{i=1}^{N_{S}} v\left(\mathbf{r}-\mathbf{r}_{i}\right), \\
& v(\mathbf{r}) \propto \frac{1}{2 \pi \sigma^{2}} e^{-|\mathbf{r}|^{2} / 2 \sigma^{2}},
\end{aligned}
$$

the correlation function becomes Gaussian,

$$
\overline{V(\mathbf{r}) V\left(\mathbf{r}^{\prime}\right)} \propto \frac{1}{2 \pi \sigma^{2}} e^{-\left|\mathbf{r}-\mathbf{r}^{\prime}\right|^{2} / 2 \sigma^{2}}
$$

The concentration necessary to reach the high concentration limit is much lower for Gaussian scatterers than for $\delta$-scatterers. The number of impurities an electron can feel increases with the range of the scatterers so that for Gaussian scatterers the effective impurity concentration is enhanced by a factor $1+\sigma^{2} / l_{c}^{2}$ relative to $\delta$-scatterers (Ando and Aoki, 1985). For scatterers

of the same strength $V$ the bandwidth is reduced by a factor of $\beta=\left(1+\sigma^{2} / l_{c}^{2}\right)^{1 / 2}$ relative to the $\delta$-scatterers, $\Gamma_{\sigma}=\Gamma / \beta$ (Ando and Uemura, 1974).

Choosing different numbers of attractive and repulsive scatterers allows for the study of potentials that are not particle-hole symmetric on average. A potential with only repulsive scatterers was used by Huo et al. (1993) to show that in this case the critical energy is not simply related to features in the density of states.

\section{B. Landau-Space Models}

This section gives an overview over models that specify the disorder distribution in the space of the Landau states. While it provides the reader with the basic ideas and results a 
more detailed description is given in the Appendix $\mathrm{X}$.

In a strong magnetic field it is helpful to choose a set of basis states in which the kinetic energy part of the Hamiltonian $H_{0}$ (3.2) is diagonal. In the Landau gauge

$$
\mathbf{A}=B x \hat{\mathrm{e}}_{y}
$$

and for a strip geometry of width $L_{y}$ with periodic boundary conditions in the $y$-direction these states are the Landau states

$$
\psi_{n k}(\mathbf{r}) \equiv\langle\mathbf{r} \mid n k\rangle=\frac{1}{\sqrt{L_{y} l_{c}}} e^{i k y} \chi_{n}\left(\frac{x-k l_{c}^{2}}{l_{c}}\right)
$$

where

$$
\chi_{n}(x)=\left(2^{n} n ! \sqrt{\pi}\right)^{-1 / 2} H_{n}(x) e^{-x^{2} / 2}
$$

are the harmonic-oscillator eigenfunctions, and $H_{n}(x)$ are Hermite polynomials. In terms of these states the Hamiltonian including the disorder potential $V(\mathbf{r})$ can be written as

$$
\begin{gathered}
H=\sum_{n k} \sum_{n^{\prime} k^{\prime}}|n k\rangle\left\langle n k|H| n^{\prime} k^{\prime}\right\rangle\left\langle n^{\prime} k^{\prime}\right|, \\
\left\langle n k|H| n^{\prime} k^{\prime}\right\rangle=\left(n+\frac{1}{2}\right) \hbar \omega_{\mathrm{c}} \delta_{n, n^{\prime}} \delta_{k, k^{\prime}}+\left\langle n k|V| n^{\prime} k^{\prime}\right\rangle .
\end{gathered}
$$

For a particular realization of the real-space potential, the matrix elements $\left\langle n k|V| n^{\prime} k^{\prime}\right\rangle$ can now be calculated. Instead, one could try to calculate the distribution function of the matrix elements given a particular distribution of the real space potential. However, to study the universal critical behavior of the Hamiltonian it is not necessary to know the whole distribution. The universal properties presumably only depend on a few statistical properties of the Hamiltonian, like the correlation function

$$
f\left(\mathbf{r}, \mathbf{r}^{\prime}\right)=\overline{V(\mathbf{r}) V\left(\mathbf{r}^{\prime}\right)}
$$

Under this assumption it is not necessary to construct the entire distribution function of the matrix elements. By the same argument only the second moment of the matrix elements is 
important for the critical behavior. Thus it suffices to construct a random matrix $\left\langle n k|V| n^{\prime} k^{\prime}\right\rangle$ [which we will call a random Landau matrix (RLM)] with a second moment corresponding to (3.18). The correlation function for the matrix elements can be calculated from the real-space correlation function $f\left(\mathbf{r}, \mathbf{r}^{\prime}\right)$

$$
\begin{aligned}
& \overline{\left\langle n_{1} k_{1}|V| n_{2} k_{2}\right\rangle\left\langle n_{3} k_{3}|V| n_{4} k_{4}\right\rangle} \\
& =\int d^{2} r d^{2} r^{\prime}\left\langle n_{1} k_{1} \mid \mathbf{r}\right\rangle\left\langle\mathbf{r} \mid n_{2} k_{2}\right\rangle\left\langle n_{3} k_{3} \mid \mathbf{r}^{\prime}\right\rangle\left\langle\mathbf{r}^{\prime} \mid n_{4} k_{4}\right\rangle \overline{V(\mathbf{r}) V\left(\mathbf{r}^{\prime}\right)}, \\
& =\int d^{2} r d^{2} r^{\prime} \psi_{n_{1} k_{1}}^{*}(\mathbf{r}) \psi_{n_{2} k_{2}}(\mathbf{r}) \psi_{n_{3} k_{3}}^{*}\left(\mathbf{r}^{\prime}\right) \psi_{n_{4} k_{4}}\left(\mathbf{r}^{\prime}\right) f\left(\mathbf{r}, \mathbf{r}^{\prime}\right) .
\end{aligned}
$$

If the real-space correlation function is translationally invariant,

$$
f\left(\mathbf{r}, \mathbf{r}^{\prime}\right)=g\left(\left|\mathbf{r}-\mathbf{r}^{\prime}\right|\right)
$$

Eq. (3.19) simplifies to

$$
\begin{aligned}
\overline{\left\langle n_{1} k_{1}|V| n_{2} k_{2}\right\rangle\left\langle n_{3} k_{3}|V| n_{4} k_{4}\right\rangle}= & \frac{1}{L_{y}^{2} l_{c}^{2}} \delta_{k_{1}-k_{2}, k_{4}-k_{3}} \int d \rho_{x} \bar{g}\left(\rho_{x}, k_{1}-k_{2}\right) \int d X \\
& \times \chi_{n_{1}}\left(\frac{X+\rho_{x} / 2-k_{1} l_{c}^{2}}{l_{c}}\right) \chi_{n_{2}}\left(\frac{X+\rho_{x} / 2-k_{2} l_{c}^{2}}{l_{c}}\right) \\
& \times \chi_{n_{3}}\left(\frac{X-\rho_{x} / 2-k_{3} l_{c}^{2}}{l_{c}}\right) \chi_{n_{4}}\left(\frac{X-\rho_{x} / 2-k_{4} l_{c}^{2}}{l_{c}}\right),
\end{aligned}
$$

where $\bar{g}(x, k)$ is the Fourier transform of $g(x, y)$ with respect to $y$,

$$
\bar{g}(x, k)=\int_{-L_{y} / 2}^{L_{y} / 2} d y g(x, y) e^{i k y}
$$

The $\delta$-function in Eq. (3.21) reflects the translational invariance of the correlation function. Equation (3.21) describes the statistical properties of the RLM. For magnetic fields much stronger than the disorder potential the coupling between the states within one Landau level becomes much stronger than the coupling between states in different Landau levels. Neglecting the coupling between different Landau levels leads to the single-band approximation in which the RLM becomes block diagonal in the Landau level indices $n_{i}$. In this approximation the physics of each Landau level can be discussed independently. For simplicity, let us restrict our discussion of the statistical properties of the RLM in the single-band 
approximation to the lowest Landau level $n=0$ and the case of a random potential with Gaussian correlations

$$
g(\mathbf{r})=\frac{V_{0}^{2}}{2 \pi \sigma^{2}} \exp \left(-\frac{|\mathbf{r}|^{2}}{2 \sigma^{2}}\right) .
$$

The correlation function (3.21) then becomes (up to terms vanishing exponentially in the $\left.\operatorname{limit} L_{y} \rightarrow \infty\right)$

$$
\begin{aligned}
& \overline{\left\langle 0 k_{1}|V| 0 k_{2}\right\rangle\left\langle 0 k_{3}|V| 0 k_{4}\right\rangle}=\frac{V_{0}^{2}}{2 \sqrt{2 \pi} \sigma L_{y}} \delta_{k_{1}-k_{2}, k_{4}-k_{3}} \exp \left(-\frac{\left(k_{1}-k_{2}\right)^{2} \sigma^{2}}{2}\right) \int d x \int d x^{\prime} \\
& \times \exp \left(-\frac{\left(x-x^{\prime}\right)^{2}}{2 \sigma^{2}}\right) \chi_{0}\left(\frac{x-k_{1} l_{c}^{2}}{l_{c}}\right) \chi_{0}\left(\frac{x-k_{2} l_{c}^{2}}{l_{c}}\right) \chi_{0}\left(\frac{x^{\prime}-k_{3} l_{c}^{2}}{l_{c}}\right) \chi_{0}\left(\frac{x^{\prime}-k_{4} l_{c}^{2}}{l_{c}}\right), \\
& =\frac{V_{0}^{2}}{\sqrt{2 \pi} l_{c} L_{y} \beta} \exp \left(-\frac{1}{2}\left(k_{1}-k_{2}\right)^{2} l_{c}^{2} \beta^{2}\right) \exp \left(-\frac{1}{2}\left(k_{1}-k_{4}\right)^{2} l_{c}^{2} \frac{1}{\beta^{2}}\right) \delta_{k_{1}-k_{2}, k_{4}-k_{3}},
\end{aligned}
$$

where $\beta^{2}=\left(\sigma^{2}+l_{c}^{2}\right) / l_{c}^{2}$ characterizes the correlation length of the potential projected onto the lowest Landau level relative to the magnetic length. Due to the Kronecker $\delta$ in Eq. (3.24) the matrix elements $\left\langle 0 k_{1}|V| 0 k_{2}\right\rangle$ are correlated only if they belong to the same diagonal $k_{1}-k_{2}=$ const. More precisely, if $\left\langle 0 k_{1}|V| 0 k_{2}\right\rangle$ is split into its real and imaginary parts, $\left\langle 0 k_{1}|V| 0 k_{2}\right\rangle_{\mathrm{R}}$ and $\left\langle 0 k_{1}|V| 0 k_{2}\right\rangle_{\mathrm{I}}$, respectively,

$$
\left\langle 0 k_{1}|V| 0 k_{2}\right\rangle=\left\langle 0 k_{1}|V| 0 k_{2}\right\rangle_{\mathrm{R}}+i\left\langle 0 k_{1}|V| 0 k_{2}\right\rangle_{\mathrm{I}},
$$

then, due to hermiticity,

$$
\begin{aligned}
\overline{\left\langle 0 k_{1}|V| 0 k_{2}\right\rangle_{\mathrm{R}}\left\langle 0 k_{3}|V| 0 k_{4}\right\rangle_{\mathrm{R}}} & =\frac{1}{2}\left(\delta_{k_{1}-k_{2}, k_{4}-k_{3}}+\delta_{k_{1}-k_{2}, k_{3}-k_{4}}\right) p\left(0,0,0,0 ; k_{1}, k_{2}, k_{3}, k_{4}\right), \\
\overline{\left\langle 0 k_{1}|V| 0 k_{2}\right\rangle_{\mathrm{I}}\left\langle 0 k_{3}|V| 0 k_{4}\right\rangle_{\mathrm{I}}} & =\frac{1}{2}\left(\delta_{k_{1}-k_{2}, k_{4}-k_{3}}-\delta_{k_{1}-k_{2}, k_{3}-k_{4}}\right) p\left(0,0,0,0 ; k_{1}, k_{2}, k_{3}, k_{4}\right),
\end{aligned}
$$

where $p\left(0,0,0,0 ; k_{1}, k_{2}, k_{3}, k_{4}\right)$ is the correlation function of Eq. (3.24) without the Kronecker $\delta$, and real and imaginary parts are uncorrelated (Huckestein and Kramer, 1989). The first exponential factor in Eq. (3.24) leads to a band structure in the RLM, i.e., as $k_{1}-k_{2}$ becomes large the average magnitude of the matrix element $\overline{\left|\left\langle 0 k_{1}|V| 0 k_{2}\right\rangle\right|^{2}}$ becomes exponentially small. The second exponential factor governs the strength of the correlations along the diagonals. Note that even for $\sigma \rightarrow 0$, i.e., for $\delta$-correlated potential, the range of the correlations does not vanish due to the Gaussian spread of the Landau wavefunctions. 
We now want to express the correlated matrix elements $\left\langle n_{1} k_{1}|V| n_{2} k_{2}\right\rangle$, which satisfy the correlation function (3.21), in terms of uncorrelated random variables, that can relatively easily be generated on a computer. The correlation function motivates the ansatz

$$
\begin{aligned}
\left\langle n_{1} k_{1}|V| n_{2} k_{2}\right\rangle= & \frac{1}{L_{y} l_{c}} \int d x d x^{\prime} h\left(x-x^{\prime}, k_{1}-k_{2}\right) u_{0}\left(x^{\prime}, k_{1}-k_{2}\right) \\
& \times \chi_{n_{1}}\left(\frac{x-k_{1} l_{c}^{2}}{l_{c}}\right) \chi_{n_{2}}\left(\frac{x-k_{2} l_{c}^{2}}{l_{c}}\right),
\end{aligned}
$$

where the $u_{0}(x, k)$ are uncorrelated, complex random variables satisfying

$$
\overline{u_{0}(x, k) u_{0}\left(x^{\prime}, k^{\prime}\right)}=\delta\left(x-x^{\prime}\right) \delta_{k,-k^{\prime}} .
$$

For the simple case of Gaussian correlations the intra-Landau-level matrix elements in the lowest Landau level are given by

$$
\begin{aligned}
\left\langle 0 k_{1}|V| 0 k_{2}\right\rangle= & \frac{V_{0}}{\sqrt{2 \pi L_{y}}} \exp \left(\frac{\left(k_{1}-k_{2}\right)^{2} l_{c}^{2} \beta^{2}}{4}\right) \\
& \times \int d \xi u_{0}\left(\beta \xi+\frac{\left(k_{1}+k_{2}\right) l_{c}}{2},\left(k_{1}-k_{2}\right) l_{c}\right) \exp \left(-\xi^{2}\right) .
\end{aligned}
$$

Note that this is also the form of Eq. (A6) for any Landau level $n_{1}=n_{2}$ in the limit $\sigma / l_{c} \rightarrow \infty$ (Huckestein, 1992)

It is worthwhile to compare the RLM model with the tight-binding model of section IIIC. The correlation function in (3.21) describes a random Hamiltonian on a one-dimensional lattice where the lattice sites are labeled by the integers $i=k L_{y} / 2 \pi$. The diagonal matrix elements $\langle n k|V| n k\rangle$ correspond to the random site energies $\epsilon_{i}$. The differences to the Anderson Hamiltonian are in the off-diagonal matrix elements that correspond to the hopping matrix elements $V_{i j}$. While in the Anderson case the off-diagonal matrix elements are real constants and couple only nearest neighbors, they are random complex variables in the RLM that couple all sites within the range $|i-j| \approx L_{y} / 2 \pi l_{c} \beta$. As is shown in section $\nabla I I$

\footnotetext{
${ }^{1}$ In one dimension the hopping matrix elements can be made real by a global gauge transformation since closed paths can enclose no flux.
} 
for finite $L_{y}$, the RLM has only localized states. However, the largest localization length is of the order of $L_{y}$ and in the two-dimensional limit, $L_{y} \rightarrow \infty$, both the range of the hopping matrix elements and the maximum localization length diverge.

\section{Lattice Models}

The standard model of localization is Anderson's tight-binding Hamiltonian (Anderson, 1958)

$$
H=\sum_{i} \epsilon_{i}|i\rangle\left\langle i\left|+\sum_{\{i, j\}} V_{i j}\right| i\right\rangle\langle j|
$$

where $i$ labels the sites on a lattice and $\{i, j\}$ are nearest neighbors. The site energies $\epsilon_{i}$ are independent random variables and in the absence of a magnetic field the hopping matrix elements are constant, $V_{i j}=V$, and define the energy scale. With this Hamiltonian the Schrödinger equation takes the form

$$
\epsilon_{i} a_{i}+\sum_{\substack{j \\\{i, j\}}} V_{i j} a_{j}=E a_{i}
$$

where $a_{i}$ is the amplitudes of the wave function at site $i$. For $\epsilon_{i}=0$ the Hamiltonian describes an electron moving in a perfect crystal.

This model can be understood as the simplest form of a more general tight binding model where the $V_{i j}$ are a function of $i$ and $j$. Such a model can be derived from the Schrödinger equation for a free particle in a periodic potential by expanding the wavefunction in terms of a complete set of orthonormal Wannier functions localized at each atomic site (Thouless, 1974)

$$
\psi(\mathbf{r})=\sum_{i} \sum_{n} a_{i}^{(n)} \phi^{(n)}\left(\mathbf{r}-\mathbf{R}_{i}\right)
$$

where $\mathbf{R}_{i}$ is the position of atom $i$ and $n$ labels the atomic orbitals. Taking into account only a single orbital per site leads to an equation of the form (3.32). 
In two dimensions on a square lattice, Eq. (3.32) has eigenvalues $E$ in the band between $-4 \mathrm{~V}$ and $+4 \mathrm{~V}$. In the presence of disorder all states in the system are localized (Abrahams et al., 1979; Wegner, 1979; MacKinnon and Kramer, 1981).

When a magnetic field is applied the Hamiltonian (3.31) has to be modified. To lowest order in the magnetic field the influence of the magnetic field on the Wannier functions can be neglected and the effect of the magnetic field is to change the phase of the wavefunction between two sites. This is achieved by replacing the constant hopping matrix elements by

$$
V_{i j}=V \exp \left(-\frac{i e}{\hbar} \int_{\mathbf{r}_{i}}^{\mathbf{r}_{j}} d r \mathbf{A}(\mathbf{r})\right)
$$

a procedure known as Peierls substitution (Peierls, 1933; Luttinger, 1951). In the absence of disorder this system shows a very rich band structure, the self-similar "Hofstadter butterfly" (Azbel', 1964; Hofstadter, 1976). In particular, if the number of flux quanta per unit cell of the lattice, $a^{2} / 2 \pi l_{c}^{2}$, where $a$ is the lattice constant, is a rational number $p / q$, the tight binding band splits into $q$ magnetic subbands.

In the presence of disorder this model can quite accurately describe the lowest Landau levels of a continuum model. This is the case in an intermediate range of the disorder where the disorder broadening of the magnetic subbands is large compared to their intrinsic width but small compared to the separation of the magnetic subbands. Schweitzer et al. (1984) have shown by a recursive Green function method that in such a situation the density of states of the lowest magnetic subband agrees well with the exact result Eq. (3.7) for the continuum model. It should be noted that this correspondence between the tight binding model and free electrons holds only for the lowest magnetic subbands near the edges of the spectrum. 


\section{Semiclassical Approximation and Network Model}

For potentials smooth on the scale of the magnetic length $l_{c}$ the localization in the integer QHE can be discussed in terms of semiclassical quantization and percolation.? These ideas have been developed by Tsukada (1976), Iordansky (1982), Kazarinov and Luryi (1982), Ono (1982a), Prange and Joynt (1982), Trugman (1983), Shapiro (1986), Wilkinson (1987), Chalker and Coddington (1988), Fertig (1988), Mil'nikov and Sokolov (1988), Jaeger (1991), and Lee, Wang, and Kivelson (1993). In the semiclassical limit, the motion of the electron separates into two components with vastly different time and length scales. On the one hand there is the slow motion of the guiding centers of the cyclotron orbits along equipotential lines of the disorder potential that is governed by classical drift equations. On the other hand there is the rapid cyclotron motion of frequency $\omega_{c}$ around the guiding center within the classical cyclotron radius $R_{c}=\sqrt{2 n+1} l_{c}$ from the guiding center. The wavefunctions are non-zero on strips of width $R_{c}$ around the path of the guiding center. They are thus located at the edges of the regions where $V(\mathbf{r})<E$ (see Fig. 8). Near minima of the potential the wavefunctions encircle these minima, while near maxima of the potential they encircle the maxima. In both cases the orbits are closed and the states localized. Since $V(\mathbf{r})$ is a random potential the properties of the regions $V(\mathbf{r})<E$ are described by the continuum model of percolation (Trugman, 1983) with the regions bounded by the equipotential lines corresponding to the clusters of the percolation model. At one energy between the minima and maxima the largest percolation cluster extends throughout the whole system and at this energy the wave functions are extended. For a disorder potential with a distribution that is symmetric this energy corresponds to the band center.

The rapid cyclotron motion around the guiding centers and hence the extent of the wavefunctions perpendicular to the equipotential line become important whenever two orbits approach each other on a distance less than the cyclotron radius. This happens near saddle

\footnotetext{
${ }^{2}$ Note that an expansion in $l_{c}^{2}$ is also an expansion in powers of $\hbar$.
} 
points of the potential. There tunneling between the two orbits becomes important and effects of quantum interference can become observable. These effects are most important near the percolation threshold because there the percolating equipotential line is split up by saddle points into localized, closed contours. Thus while tunneling and quantum interference have a negligible effect in the tails of the density of states, they can in fact change the critical behavior of the system.

Chalker and Coddington (1988) created a model that captures the physics of percolation, tunneling and quantum interference near the percolation threshold by introducing a network of saddle points connected by equipotential lines. Due to its quantum nature this network model shows critical behavior that is different from the classical percolation problem but coincides with that for short-ranged potentials (see section VIII).

In the following we will first introduce the semiclassical picture of the QHE and then motivate the network model as a simplification of this picture.

The semiclassical approximation is most conveniently derived by replacing the coordinates $(x, y)$ by guiding center coordinates $(X, Y)$ and relative coordinates $(\xi, \eta)$, given by

$$
\begin{aligned}
& x=X+\xi, \\
& y=Y+\eta,
\end{aligned}
$$

and

$$
\begin{aligned}
& \xi=-\frac{1}{e B}\left(p_{y}-e A_{y}\right), \\
& \eta=\frac{1}{e B}\left(p_{x}-e A_{x}\right) .
\end{aligned}
$$

From the equations of motion for the pure system $(V(\mathbf{r})=0)$, we get

$$
\begin{aligned}
& \dot{\xi}=\frac{i}{\hbar}\left[H_{0}, \xi\right]=\omega_{c} \eta, \\
& \dot{\eta}=\frac{i}{\hbar}\left[H_{0}, \eta\right]=-\omega_{c} \xi
\end{aligned}
$$


We see that $(\xi, \eta)$ indeed rotate with angular frequency $\omega_{c}$ around the guiding center. Due to the commutation relations of $\mathbf{p}$ and $\mathbf{r}$ both the guiding center coordinates and the relative coordinates obey canonical commutation relations,

$$
\begin{gathered}
{[\xi, \eta]=i l_{c}^{2},} \\
{[X, Y]=-i l_{c}^{2} .}
\end{gathered}
$$

With Eq. (3.36), the Hamiltonian $H_{0}$ can be written in terms of $\xi$ and $\eta$,

$$
H_{0}=\frac{\hbar \omega_{c}}{2 l_{c}^{2}}\left(\xi^{2}+\eta^{2}\right),
$$

which expresses the degeneracy of the Landau levels as $H_{0}$ does not depend on $X$ and $Y$.

In the presence of a disorder potential $V(\mathbf{r})$ the degeneracy is lifted. The equations of motion for the center coordinates are

$$
\begin{aligned}
& \dot{X}=\frac{i}{\hbar}[H, X]=-\frac{l_{c}^{2}}{\hbar} \frac{\partial V}{\partial y}, \\
& \dot{Y}=\frac{i}{\hbar}[H, Y]=\frac{l_{c}^{2}}{\hbar} \frac{\partial V}{\partial x} .
\end{aligned}
$$

If the potential $V(\mathbf{r})$ is smooth on the scale $l_{c}$, we can replace $V(x, y)$ by $V(X, Y)$ and obtain a drift of the guiding center along equipotentials,

$$
\begin{aligned}
& \dot{X}=-\frac{l_{c}^{2}}{\hbar} \frac{\partial V}{\partial Y}, \\
& \dot{Y}=\frac{l_{c}^{2}}{\hbar} \frac{\partial V}{\partial X} .
\end{aligned}
$$

In this limit, the eigenenergies are

$$
E=(n+1 / 2) \hbar \omega_{c}+V(X, Y) .
$$

Due to the breaking of the time reversal symmetry by the magnetic field the guiding centers can move only in one direction determined by the magnetic field and the slope of the potential (Fig. 8).

The electron eigenfunctions can be approximated by 


$$
\psi(u, v)=C(u) \chi_{n}(v) e^{i \phi(u, v)},
$$

where the variables $u$ and $v$ parametrize the distance along and perpendicular to the equipotential line, respectively,

$$
C^{2}(u) \propto \frac{1}{|\nabla V(u, v)|_{v=0}},
$$

and $\phi(u, v)$ is a gauge-dependent phase (Trugman, 1983). The semiclassical quantization condition is that the phase $\phi(u, v)$ must change by a multiple of $2 \pi$ upon going around a closed contour. This condition determines the allowed energies. From Eq. (3.43) it follows that the overlap between different states is exponentially small as long as the separation between the corresponding contours is large compared to the magnetic length.

The problem of finding the wavefunction of largest extent is equivalent to the problem of finding the largest connected cluster in a continuum percolation problem (Stauffer, 1979). The "diameter" $\xi_{P}(E)$ of this cluster grows on approaching the percolation threshold $E_{c}$ as

$$
\xi_{P}(E) \propto\left|E-E_{c}\right|^{-\nu_{P}},
$$

where the percolation critical exponent $\nu_{P}$ is $4 / 3$ (den Nijs, 1979; Black and Emery, 1981).

In addition to the semiclassical drift of the guiding center coordinates tunneling has to be considered near saddle points of the potential, where two orbits approach each other. In order to study the influence of tunneling on percolation Chalker and Coddington(1988) introduced a network model. To motivate this model, let us look at an electron moving along an equipotential line far away, compared to $l_{c}$, from other trajectories. Then its wavefunction is given by Eq. (3.43). This wavefunction also contains information about the degrees of freedom perpendicular to the trajectory that we are not interested in. In its place, we consider a simpler quantity, the complex function $Z(u)$ of the coordinate $u$ along the equipotential line, defined by

$$
\arg (Z(u))=\arg (\Psi(u, v=0))
$$

and 


$$
|Z(u)|^{2}=\int d v \psi^{*}(u, v) \hat{j}_{u} \psi(u, v)
$$

where $\hat{j}_{u}$ is the $u$-component of the current density operator and $u$ increases in the direction of net current flow so that the integral is positive. Since no current can escape perpendicular to the equipotential line $Z(u)$ can only change its phase along the trajectory. This phase change depends on the gauge and arc-length of the trajectory in units of $l_{c}$.

Let us now consider the region near the saddle points (Fig. 9). There two degenerate wavefunctions meet and, due to tunneling, the description in terms of the $Z(u)$ breaks down. Let us denote by $Z_{1}, Z_{2}, Z_{3}$, and $Z_{4}$ the values of $Z(u)$ on the four arms some distance large compared to $l_{c}$ away from the saddle point, where the $Z(u)$ are still fair descriptions of the exact eigenfunctions. Due to the directedness of the trajectories two of these describe ingoing currents (say, $Z_{1}$ and $Z_{2}$ ) and two describe outgoing $\left(Z_{3}\right.$ and $Z_{4}$ ). Solving the Schrödinger equation near the saddle point gives a relation between the ingoing and outgoing currents,

$$
\left(\begin{array}{l}
Z_{1} \\
Z_{3}
\end{array}\right)=M\left(\begin{array}{l}
Z_{4} \\
Z_{2}
\end{array}\right) .
$$

Current conservation constrains the $2 \times 2$ matrix $M$ to unitarity $\left|Z_{1}\right|^{2}+\left|Z_{2}\right|^{2}=\left|Z_{3}\right|^{2}+\left|Z_{4}\right|^{2}$. This implies

$$
J=M^{\dagger} J M
$$

where

$$
J=\left(\begin{array}{cc}
1 & 0 \\
0 & -1
\end{array}\right)
$$

which has the general solution

$$
M=\left(\begin{array}{cc}
e^{i \phi_{1}} & 0 \\
0 & e^{i \phi_{2}}
\end{array}\right)\left(\begin{array}{cc}
\cosh \theta & \sinh \theta \\
\sinh \theta & \cosh \theta
\end{array}\right)\left(\begin{array}{cc}
e^{i \phi_{3}} & 0 \\
0 & e^{i \phi_{4}}
\end{array}\right),
$$

with real $\theta, \phi$. The phases $\phi_{i}$ can be set to zero by a suitable choice of gauge so that the saddle point is completely characterized by a real parameter $\theta$. For $\theta \ll 1$ the saddle point behaves classically and the electron almost always goes from 1 to 4 : $\left|Z_{1}\right|^{2} \approx\left|Z_{4}\right|^{2}$. For $\theta \gg 1$ 
it also behaves classically, but now 1 goes to $3:\left|Z_{1}\right|^{2} \approx\left|Z_{3}\right|^{2}$. In between the saddle point behaves quantum mechanically. For a special value $\theta_{c}$ the saddle point will be symmetric. At this value the probability to scatter from 1 to 3 and to 4 are the same. $\theta_{c}$ is given by

$$
\sinh \theta_{c}=1
$$

which has the solution $\theta_{c}=\ln (1+\sqrt{2}) \approx 0.8814$. Fertig (1988) showed how $\theta$ depends on the energy,

$$
\sinh (\theta)=\exp (-\pi \gamma / 2)
$$

where

$$
\gamma=\frac{4\left(E-V_{1}\right)}{\left|V_{x x} V_{y y}\right|^{1 / 2} l_{c}^{2}}
$$

$V_{1}$ is the saddle point potential, and $V_{i i}$ is the second derivative of the potential in suitable coordinates.

The network model consists of a square lattice of saddle points, each described by a $\theta$ parameter (see Fig. 10). Two neighboring saddle points are connected by directed links representing the equipotentials such that at every saddle point there are two incoming and to outgoing links. Each link is characterized by the phase change of $Z(u)$ along the link. Since the lengths of the links and hence the phase change depends on the random potential these phases are taken to be uniformly distributed random variables. In the original paper (Chalker and Coddington, 1988) the $\theta$ parameter was fixed for all nodes in the network. Generalizations of the model where the $\theta$ 's were random variables were studied by Chalker and Eastmond (1993) and Lee et al. (1993).

In contrast to the other models of disorder the network model does not deal with wavefunctions but with transmission probability amplitudes. Thus it lends itself naturely to the study of properties of the transmission matrix through the system but it is not obvious how to get information about the multifractal properties of the system discussed in section IXC.

Mil'nikov and Sokolov (1988) employed the semiclassical approximation to calculate the inverse localization length $\xi(E)^{-1}$ in an intermediate energy range, $\Gamma_{\sigma}\left(l_{c} / \sigma\right)^{2} \ll E \ll \Gamma_{\sigma}$. In 
this energy range the equipotential lines differ significantly from the percolating equipotential line only near the saddle points, where the percolating equipotential line is split up. Due to the first strong inequality the parameter $\gamma$ of Eq. (3.54) is large at these saddle points and with Eqs. (3.48) and (3.53) the transmission probability through the saddle point is exponentially small. Mil'nikov and Sokolov assume that in this limit a single path, which consists of saddle points connected by parts of equipotential lines, is important for the transmission probability between points far apart in space. Then they treat this problem using the semiclassical theory of tunneling through a one-dimensional barrier. The solution to the eigenvalue equation (3.42) defines a dispersion relation $Y(E, X)$ similar to the dispersion relation of a one-dimensional particle in a potential $k(E, x)$. The solutions $Y(E, X)$ are real on the equipotential lines but are complex in the classically forbidden regions near the saddle points. With Eq. (3.42) the inverse localization length (6.2) can be written as

$$
\xi(E)^{-1}=\overline{\min _{n}\left|\operatorname{Im} Y_{n}(E)\right| / l_{c}^{2}}
$$

where $Y_{n}(E)$ are the roots of Eq. (3.42). The average distance between saddle points is given by the diameter $\xi_{P}(E)$ (3.45). Thus we can rewrite Eq. (3.55),

$$
\xi(E)^{-1}=\xi_{P}(E)^{-1} \overline{\int d X|\operatorname{Im} Y(E, X)| / l_{c}^{2}}
$$

where the integral runs over the classically forbidden range of $X$ near a saddle point. Since both the width of this region and the maximum of $|\operatorname{Im} Y(E, X)|$ are proportional to $E^{1 / 2}$, the integral in Eq. (3.56) is proportional to $E$ and with Eq. (3.45) we find

$$
\xi(E)^{-1} \propto E^{\nu_{P}+1}=E^{7 / 3} .
$$

While the result $\nu=7 / 3$ agrees with the numerical value 8.4 it is not clear whether it describes the same physical situation. The derivation of the result is based on the assumption that only a single trajectory between two points is important. While this assumption seems justified if $E \gg \Gamma_{\sigma}\left(l_{c} / \sigma\right)^{2}$ and each saddle point transmits predominantly into a single link, closer to the percolation threshold the probabilities for transmission into both possible links 
become comparable. In this energy range the two-dimensional character of the percolating equipotential line has to be taken into account. It is this limit that is described by the network model, which has (see Sec. VIIIA) the same scaling behavior as the random Landau matrix model.

\section{FINITE-SIZE SCALING}

In this section we want to review the finite-size scaling theory as it was developed for thermodynamic phase transitions. Since the transitions between quantum Hall plateaus are believed to be zero-temperature quantum phase transitions we want to analyze the numerical data in section VII1 in terms of this finite-size scaling theory. We base our discussion on the review of finite-size scaling by Barber (1983).

For infinite systems, thermodynamic quantities can diverge at phase transitions. The "usual" scaling theory describes the behavior of the thermodynamic quantities near the transition in terms of scaling laws. In finite systems there are no phase transitions and no singularities in thermodynamic quantities. Nevertheless in the simultaneous limit that the temperature approaches the critical temperature and the system size goes to infinity the scaling laws of the infinite system are reflected in scaling laws for the finite system involving the system size. It is this finite-size scaling theory that we want to focus on in this section.

\section{A. Single-Parameter Scaling}

Let us consider a $d$-dimensional thermodynamic system that is finite in at least one direction. Since we are interested in the QHE in two dimensions, let us assume $d=2$. Then the system could have the geometry of a quasi-one-dimensional strip or cylinder, depending on the boundary conditions in the transverse direction, or it could be finite in both directions. Let us further assume that the infinite $d$-dimensional system has a phase transition but that the system with the finite length scale has no transition. An example of such a system would be the Ising model. 
Let $P_{\infty}(T)$ be a thermodynamic quantity of the infinite system that diverges at the critical point $T_{c}$,

$$
P_{\infty}(T) \propto t^{-\rho}
$$

as $t=\left(T-T_{c}\right) / T_{c} \rightarrow 0$. For the $d=2$ Ising model this could be the susceptibility and the parameter $T$ is the temperature. For a finite system this divergence will be rounded and the function $P_{L}(T)$ of the finite system will have a maximum at a position $T_{m}(L)$ that will in general be different from $T_{c}$ but will approach $T_{c}$ as $L \rightarrow \infty$. The approach is characterized by a shift exponent $\lambda$ defined by

$$
\frac{T_{m}(L)-T_{c}}{T_{c}} \propto L^{-\lambda}, \quad L \rightarrow \infty
$$

where $L$ is measured in multiples of some microscopic length scale of the system.

The central idea of finite-size scaling is that the magnitude of the finite-size effects are determined by a single length scale $\xi(T)$ that diverges at the critical point,

$$
\xi(T) \propto t^{-\nu}, \quad t \rightarrow 0
$$

This length scale is identified with the correlation length of order parameter fluctuations. Thermodynamic quantities will depend on the ratio

$$
y=L / \xi(T) .
$$

For $y \gg 1$, the system does not feel the finite size and intensive quantities will not depend on $L$. For $y \lesssim 1$, the finite size rounds off the thermodynamic singularities associated with the phase transition. Thus $y=1$ defines another characteristic temperature $T^{*}(L)$ of the system where finite-size effects become important. From Eqs. (4.3,4.4) it follows that

$$
\frac{T^{*}(L)-T_{c}}{T_{c}} \propto L^{-1 / \nu}, \quad L \rightarrow \infty
$$

If $\xi(T)$ is the only relevant length scale the shift exponent $\lambda$ must also be given by $\lambda=1 / \nu$ (Barber, 1983). In the following we will not consider modifications to the finite-size scaling 
analysis necessary to deal with the shift of the "critical" point in finite systems. The systems that we want to analyze exhibit a symmetry such that the maxima of scaling functions are always located at the critical point of the infinite system.

The finite-size scaling hypothesis states that for finite $L$ and $T$ near $T_{c}$,

$$
P_{L}(T)=L^{\omega} Q(y)
$$

The exponent $\omega$ is determined by requiring that Eq. (4.6) reproduces Eq. (4.1) in the limit $L \rightarrow \infty$. This requires

$$
Q(y) \propto y^{-\omega}, \quad y \rightarrow \infty
$$

and

$$
\omega=\rho / \nu
$$

For $y \rightarrow 0$ at fixed $L, P_{L}(T)$ has to go to a finite value and hence

$$
Q(y) \rightarrow \text { const. }, \quad y \rightarrow 0
$$

For the particular case of the correlation length $\xi_{L}$ of the finite system the finite-size scaling relation (4.6) becomes

$$
\xi_{L}(T)=L Q_{\xi}(y)
$$

since comparing Eqs. (4.1) and (4.3) yields $\rho=\nu$ and hence $\omega=1$.

The phenomenological finite-size scaling theory for a particular thermodynamic quantity outlined above can be related to and derived from the scaling theory based on the renormalization group for infinite systems (Suzuki, 1977; Brézin, 1982; Barber, 1983). The renormalization group establishes a relation between the coupling constants $\mathbf{K}$ of a Hamiltonian $H$ and the coupling constants $\mathbf{K}^{\prime}$ of a Hamiltonian $H^{\prime}$, in which all lengths have been rescaled by a factor $b$ relative to $H$,

$$
\mathbf{K}^{\prime}=\mathbf{R}(\mathbf{K})
$$


where $\mathbf{R}$ is an analytic function of $\mathbf{K}$. At a phase transition there exists a non-trivial fixed point $\mathbf{K}^{*}$ of the recursion Eq. (4.11), $\mathbf{K}^{*}=\mathbf{R}\left(\mathbf{K}^{*}\right)$. Near this fixed point the transformations (4.11) can be linearized and the matrix $\partial R_{\alpha} / \partial K_{\beta}$ evaluated at $\mathbf{K}^{*}$ has the eigenvalues

$$
\lambda_{\alpha}=b^{y_{\alpha}}
$$

Using these eigenvalues Eq. (4.11) can be rewritten as

$$
\zeta_{\alpha}^{\prime}=b^{y_{\alpha}} \zeta_{\alpha}
$$

where $\zeta_{\alpha}$ is a non-linear scaling field that is a regular function of the coupling constants $\mathbf{K}$. In terms of the scaling fields the fixed point $\mathbf{K}^{\prime}=\mathbf{K}$ is given by $\zeta_{\alpha}=0$.

The singular part of the free energy per degree of freedom $f_{s}$, responsible for the divergence of thermodynamic quantities at the phase transition, can then be expressed in terms of the scaling fields $\zeta_{\alpha}$ instead of the coupling constants $\mathbf{K} . f_{s}\left(\zeta_{1}, \ldots ; L\right)$ has the scaling form

$$
f_{s}\left(\zeta_{1}, \ldots ; L\right)=L^{-d} F\left(L^{y_{1}} \zeta_{1}, \ldots\right)
$$

where $L$ is the length scale of the system that is reached after iterating the renormalization group equation (4.11) a number of times starting from the microscopic Hamiltonian, and $F$ is an analytic function of its arguments. From the free energy (4.14) the scaling behavior of other thermodynamic functions can be obtained by differentiating the free energy appropriately,

$$
P_{L}\left(\zeta_{1}, \ldots\right)=L^{\omega} \tilde{Q}\left(L^{y_{1}} \zeta_{1}, \ldots\right)
$$

This expression is equivalent, in the thermodynamic limit, to the single-parameter finite-size scaling hypothesis (4.6) if

$$
\zeta_{1}=\zeta_{t} \propto t+O\left(t^{2}\right)
$$

and 


$$
y_{1}=1 / \nu,
$$

where we have used Eq. (4.3). Furthermore, all other scaling fields $\zeta_{i}, i \geq 2$, have to be irrelevant, i.e., $y_{i}<0, i \geq 2$. We can interpret this statement in the following way. The Hamiltonian $H$ of a system depends in general on a large number of coupling constants $K_{i}$. In the representation in terms of the scaling field $\zeta_{\alpha}$ it depends on a large number of scaling fields. However, the thermodynamic properties in the limit $L \rightarrow \infty$ depend only on a small number of scaling fields $\zeta_{\alpha}$ that have positive scaling indices $y_{\alpha}$. In conventional phase transitions these are related to the temperature $t(4.16)$ and the symmetry breaking field $h$,

$$
\zeta_{h} \propto h+O\left(h t^{2}\right) .
$$

If a coupling constant $K$ shows single-parameter finite-size scaling it means that the coupling constant $K(b L)$ of the rescaled system is a function of the coupling constant $K(L)$ of the original system and the scale factor $b$ only,

$$
K(b L)=f(b, K(L)) .
$$

In the continuum limit, $b \rightarrow 1$, this property allows one to define a $\beta$ function that is a function of $K$ only,

$$
\beta(K(L))=\frac{d \ln K(L)}{d \ln L} .
$$

The fixed points of the renormalization group are given by the zeros of the $\beta$ function (4.20). Near the fixed point the $\beta$ function can be linearized,

$$
\beta=y_{K} \ln \left(K / K^{*}\right),
$$

where Eq. (4.12) was used. For $y_{K}>0\left(y_{K}<0\right)$ the fixed point is repulsive (attractive). Comparison with Eq. (4.17) shows that the localization length exponent $\nu$ is given by the inverse slope of the $\beta$ function at the critical point. 


\section{B. Corrections to Scaling}

The scaling fields $\zeta_{\alpha}$ with negative scaling indices $y_{\alpha}<0$ are irrelevant in the thermodynamic limit $L \rightarrow \infty$. However, for finite systems they lead to corrections to the asymptotic scaling laws and it can be necessary to take these into account when analyzing numerical or experimental data for finite systems. For two scaling fields, a relevant field $\zeta_{1}$ with $y_{1}>0$ and an irrelevant field $\zeta_{2}$ with $y_{2}<0$, the renormalization flow of the "coupling constants" $x_{1}=L^{y_{1}} \zeta_{1}$ and $x_{2}=L^{y_{2}} \zeta_{2}$ under increase of the length scale $L$ is depicted in Fig. 11. The arrows indicate the direction of change of $x_{i}$ when $L$ is increased. The point $x_{1}=x_{2}=0$ is the fixed point of the renormalization group. The flow corresponds to the down-hill flow near a saddle point in a potential.

Near the fixed point a thermodynamic quantity $P_{L}\left(\zeta_{1}, \zeta_{2}, \ldots\right)$ can be expanded in the $\operatorname{arguments} x_{\alpha}$. From Eq. (4.15) we get

$$
P_{L}\left(\zeta_{1}, \zeta_{2}, \ldots\right)=L^{\omega}\left(\tilde{Q}_{0}+a_{1} L^{y_{1}} \zeta_{1}+a_{2} L^{-\left|y_{2}\right|} \zeta_{2}+\ldots\right) .
$$

If the largest irrelevant scaling index $y_{2}$ is sufficiently large compared to the next leading index it might be sufficient to take only these two indices into account and one arrives at a two-parameter scaling theory. If there are other scaling indices close to $y_{2}$ it might be necessary to treat more scaling fields.

For the saddle point fixed point shown in Fig. [1] the flow along the line $\zeta_{2}=0$ away from the fixed point is described by the single-parameter scaling relation

$$
P_{L}\left(\zeta_{1}, 0\right)=L^{\omega} \tilde{Q}\left(L^{y_{1}} \zeta_{1}, 0\right)
$$

where $y_{1}=1 / \nu$. The flow along the line $\zeta_{1}=0$ towards the fixed point is also described by a single-parameter scaling relation

$$
P_{L}\left(0, \zeta_{2}\right)=L^{\omega} \tilde{Q}\left(0, L^{-\left|y_{2}\right|} \zeta_{2}\right) .
$$

Eq. (4.24) shows that the corrections to scaling due to the irrelevant scaling field $\zeta_{2}$ exhibit finite-size scaling at the fixed point $\zeta_{1}=0$. 


\section{Dynamic Scaling}

So far we have focused our discussion on the static properties of scaling quantities. We will now consider quantities varying in space and time. Analogous to Eq. (4.1), we expect in the infinite system the wave vector $q$ and frequency $\omega$ dependence

$$
P_{\infty}(T, q, \omega) \propto t^{-\rho} Q\left(q \xi(T), \omega \tau_{0}(T)\right)
$$

The relaxation time $\tau_{0}(T)$ describes the rate at which the system relaxes towards its equilibrium state. It diverges at the transition and the dynamical exponent $z$ is defined by

$$
\tau_{0}(T) \propto \xi^{z} \propto t^{-\nu z}
$$

In a finite system fluctuation on long length-scales are cut off by the system size $L$ and from Eq. (4.25) we get the dynamic finite-size scaling relation

$$
\begin{aligned}
P_{L}(T, \omega) & =L^{\omega} Q\left(L / \xi(T), \omega \tau_{0}(T)\right) \\
& =L^{\omega} \tilde{Q}\left(L^{1 / \nu} t, \omega^{-1 / \nu z} t\right) .
\end{aligned}
$$

In the limit $\omega \rightarrow 0$ we recover the static scaling relation Eq. (4.6).

\section{SCALING BEHAVIOR AND FIELD THEORY}

In this section we discuss field-theoretical approaches to the localization problem and their relation to scaling theory. While field theory in the context of the integer QHE provides a framework for a scaling analysis, it cannot at present provide quantitative results suitable

for a comparison with experiments. We will therefore restrict ourselves to an outline of the motivation and basic ideas. Thus this section lacks the technical details found in the other sections. 


\section{A. Anderson Localization}

We will start with a brief survey of the problem of Anderson localization in the absence of strong magnetic fields. For a more detailed review the reader is referred to Kramer and MacKinnon (1993).

Using prior work of Thouless and co-workers (Edwards and Thouless, 1972; Thouless, 1974; Licciardello and Thouless, 1975) and Wegner (1976), Abrahams et al. (1979) argued that the average dimensionless conductance $g=G \hbar / e^{2}$ should obey a single parameter scaling relation. They calculated the $\beta$ function of the conductance,

$$
\frac{d \ln g}{d \ln L}=\beta(g)
$$

in the limits $g \ll 1$ and $g \gg 1$. In the localized regime, $g \ll 1$, the conductance decreases exponentially with system size, $g=g_{0} \exp (-\alpha L)$ and hence

$$
\lim _{g \rightarrow 0} \beta(g) \propto \ln g
$$

For large conductance, macroscopic transport theory expresses for a $d$-dimensional "cube" of side $L$ the conductance $G$ in terms of the conductivity $\sigma$,

$$
G(L)=\sigma L^{d-2}
$$

so that

$$
\lim _{g \rightarrow \infty} \beta(g)=d-2 .
$$

Assuming a continuous $\beta$ function Abrahams et al. arrived at the flow diagram depicted in Fig. 12. The $\beta$ function has a zero only in $d>2$ dimensions. They argued that the $\beta$ function has no zero in $d=2$ dimensions since the first correction to $\beta$ in $1 / g$ is negative in perturbation theory (Langer and Neal, 1966; Abrahams et al., 1979).

The same behavior is obtained when the disordered electron problem is mapped onto a non-linear $\sigma$ model (Wegner, 1979; Efetov et al., 1980). We will briefly sketch how this model is derived. We start by representing Green functions, like 


$$
G^{+}\left(\mathbf{r}, \mathbf{r}^{\prime}, E\right)=\left\langle\mathbf{r}\left|(z-H)^{-1}\right| \mathbf{r}^{\prime}\right\rangle
$$

$z=E+i \epsilon, \epsilon>0$, as path integrals

$$
G^{+}\left(\mathbf{r}, \mathbf{r}^{\prime}, E\right)=\frac{\int D\left[\bar{\Phi}^{+}\right] D\left[\Phi^{+}\right] \bar{\Phi}^{+}(\mathbf{r}) \Phi^{+}\left(\mathbf{r}^{\prime}\right) \exp \left(\int d^{2} r \bar{\Phi}^{+}(\mathbf{r})(z-H) \Phi^{+}(\mathbf{r})\right)}{\operatorname{det}(z-H)} .
$$

The sign of $\epsilon$ ensures the convergence of the integral. To represent advanced as well as retarded Green functions we need to introduce two additional fields $\bar{\Phi}^{-}$and $\Phi^{-}$with the opposite sign of $\epsilon$. Equation (5.6) can be interpreted as the statistical average $\left\langle\bar{\Phi}^{+}(\mathbf{r}) \Phi^{+}\left(\mathbf{r}^{\prime}\right)\right\rangle$ with the partition function

$$
\mathcal{Z}=\int D\left[\bar{\Phi}^{+}\right] D\left[\Phi^{+}\right] \exp \left(\int d^{2} r \bar{\Phi}^{+}(\mathbf{r})(z-H) \Phi^{+}(\mathbf{r})\right)
$$

Higher-order Green functions are given by higher-order correlation functions of the fields.

In order to perform the average over disorder one needs to get rid of the determinant in Eq. (5.6) that depends on the disorder potential. One way of achieving this goal is the use of the replica trick (Wegner, 1979; Schäfer and Wegner, 1980; Efetov et al., 1980). Here the fields are replicated $m$ times so that the denominator becomes $[\operatorname{det}(z-H)]^{m}$. In the formal limit $m \rightarrow 0$, performed at the end of the calculation, the determinant becomes unity. Instead, the supersymmetry method (Efetov, 1983) can be employed where commuting as well as anti-commuting (Grassmann) fields are used. Then the determinants for the commuting and anti-commuting fields cancel exactly.

Using one of these schemes, the average over disorder in Eq. (5.7) can readily be performed for the Gaussian white-noise potential (3.5). Since the disorder potential couples two fields this leads to a term containing a product of four fields in the exponential. These quartic terms can be decoupled by a Hubbard-Stratonovic transformation to matrix fields $Q(\mathbf{r})$. The effective Lagrangian for this matrix is (Pruisken and Schäfer, 1981)

$$
L[Q]=\int d^{2} r\left[-\frac{1}{2 V_{0}^{2}} \operatorname{Tr} Q^{2}(\mathbf{r})+\operatorname{Tr} \ln \left(E-H_{0}-Q\right)\right],
$$

where we dropped convergence-ensuring terms. This equation is the starting point of the derivation of the non-linear $\sigma$ model. It proceeds by evaluating the integral in Eq. (5.8) in 
saddle-point approximation, suitably decomposing the $Q$-matrix, and expanding the trace of the logarithm. The result is an effective Lagrangian

$$
L(\tilde{Q})=\int d^{2} r\left[-\frac{1}{t} \operatorname{Tr}(\partial \tilde{Q})^{2}\right]
$$

where the coupling constant $1 / t$ is proportional to the conductance.

The symmetry of the matrices $Q$ depends on the symmetry of the original Hamiltonian and the choice of commuting or anti-commuting variables.

For the non-linear $\sigma$ model (5.9) the $\beta$ function for the coupling constant and hence for the conductivity can be calculated (Brézin et al., 1980). The results depend on the symmetry class of the $Q$-fields. One distinguishes three universality classes: The orthogonal class for random potential scattering, the symplectic class in the presence of spin-orbit coupling and the unitary class in the presence of a magnetic field. The results of Wegner (1989) may be written in terms of the dimensionless conductance $g$ as follows:

$$
\beta(g)= \begin{cases}(d-2)-g^{-1}-\frac{3}{4} \zeta(3) g^{-4}+\mathcal{O}\left(g^{-5}\right), & \text { orthogonal case } \\ (d-2)-2 g^{-2}-6 g^{-4}+\mathcal{O}\left(g^{-6}\right), & \text { unitary case } \\ (d-2)+g^{-1}-\frac{3}{4} \zeta(3) g^{-4}+\mathcal{O}\left(g^{-5}\right), & \text { symplectic case }\end{cases}
$$

Only in the symplectic case does the $\beta$ function have a zero in $d=2$ dimensions while in the other cases all states are localized. In particular, the presence of a magnetic field in the unitary case does not lead to extended states necessary for explaining the QHE. We will come back to this problem in the next section.

Further support for the scaling picture of Abrahams et al. (1979) comes from numerical calculations (MacKinnon and Kramer, 1981; Pichard and Sarma, 1981; MacKinnon and Kramer, 1983). For a recent review see Kramer and MacKinnon (1993). Numerically it is easier to calculate the localization length instead of the conductance. This can be done recursively for strips or bars using either the Green-function method of section VIA or the transfer-matrix method of section $\nabla \mathrm{TB}$. The numerical results are compatible with the finite-size scaling hypothesis (4.10). In one-dimensional systems no extended states are observed. In two-dimensional systems a transition is only observed in the presence of 
spin-orbit scattering. In three-dimensional system a localization-delocalization transition is observed at a critical disorder.

After the discovery of the absence of self-averaging in mesoscopic systems (Anderson et al., 1980; Altshuler, 1985; Lee and Stone, 1985) it became apparent that the description of the metal-insulator transition in terms of the average conductance is incomplete. Altshuler, Kravtsov, and Lerner $(1986 ; 1989)$ argued that the whole distribution of the conductance needs to be considered. Single-parameter scaling in this context was discussed by Shapiro (1987). His scaling parameter need not necessarily be the average or typical conductance but any parameter that uniquely determines the distribution function.

\section{B. Nonlinear Sigma Model and Topological Term}

In the previous section it was pointed out that the absence of extended states in the nonlinear $\sigma$ model for the unitary two-dimensional case apparently contradicts the explanations for the QHE (Aoki and Ando, 1981; Laughlin, 1981; Halperin, 1982) which require the

existence of extended states for sufficiently small disorder. Pruisken (1984) and collaborators (Levine et al., 1983; Levine et al., 1984a; Levine et al., 1984b; Levine et al., 1984c) argued that the failure of the non-linear $\sigma$ model is due to the emergence of a topological term in the effective Lagrangian that is not accessible to the perturbative treatment that leads to the Lagrangian (5.9). A review of the discussion can be found in Pruisken's chapter in the book by Prange and Girvin (1987). Here we want to sketch the main features of this field theory.

The Lagrangian (5.9) has to be augmented by a term proportional to the Hall conductivity,

$$
\mathcal{L}=\int d^{2} r\left(-\frac{1}{8} \sigma_{x x}^{0} \operatorname{Tr}(\partial \tilde{Q})^{2}+\frac{1}{8} \sigma_{x y}^{0} \operatorname{Tr} \tilde{Q}\left[\partial_{x} \tilde{Q}, \partial_{y} \tilde{Q}\right]\right)
$$

The values of the coupling constants $\sigma_{x x}^{0}$ and $\sigma_{x y}^{0}$ in (5.11) are the mean-field values corresponding to the conductivity tensor on short length scales. They are given by the SCBA 
(Ando et al., 1975). Upon increase of the length scale the conductivities get renormalized to their physical values on long length scales. We will use the correspondence between the scaling on short length scales and the SCBA in our discussion of the corrections to scaling in Sec. (VIIIB).

The occurrence of two coupling constants $\sigma_{x x}^{0}$ and $\sigma_{x y}^{0}$ in the Lagrangian leads to a coupling of the renormalizations of $\sigma_{x x}$ and $\sigma_{x y}$ and changes the critical behavior compared to the non-linear $\sigma$ model for Anderson localization. In a dilute-instanton-gas approximation for the physically relevant field configurations in the weak-coupling limit $\sigma_{x x} \gg 1$ Pruisken derived $\beta$ functions for the renormalization with system size $L$ of both $\sigma_{x x}$ and $\sigma_{x y}$,

$$
\begin{aligned}
& \beta_{x x}=\frac{\partial \sigma_{x x}}{\partial \ln L}=\frac{-1}{2 \pi^{2} \sigma_{x x}}-\sigma_{x x} D e^{-2 \pi \sigma_{x x}} \cos \left(2 \pi \sigma_{x y}\right), \\
& \beta_{x y}=\frac{\partial \sigma_{x y}}{\partial \ln L}=-\sigma_{x x} D e^{-2 \pi \sigma_{x x}} \sin \left(2 \pi \sigma_{x y}\right) .
\end{aligned}
$$

This leads to the renormalization-group flow diagram proposed by Khmel'nitskii (1983) shown in Fig. 13. It has two kinds of fixed points: stable fixed points at $\sigma_{x x}=0, \sigma_{x y}=n$ and fixed points at half-integer values of $\sigma_{x y}$ with a finite conductivity $\sigma_{x x}^{*}$. The former fixed points correspond to the localized wave functions of the model in the absence of the topological term while the latter correspond to the extended wave functions that carry the current in the QHE. Furthermore the flow diagram is periodic in $\sigma_{x y}$ so that the critical behavior is independent of Landau level index, since (Levine et al., 1983)

$$
\frac{\sigma_{x y}^{0}}{8} \int d^{2} r \operatorname{Tr} \tilde{Q}\left[\partial_{x} \tilde{Q}, \partial_{y} \tilde{Q}\right]=2 \pi i q \sigma_{x y}^{0} .
$$

The delocalization fixed points at half-integer $\sigma_{x y}$ are partly attractive and partly repulsive like the fixed point discussed in connection with Fig. 11. They are characterized by a relevant scaling index $y_{1}=1 / \nu>0$ associated with the deviation from $\sigma_{x y}^{*}=(2 n+1) / 2$ and an irrelevant scaling index $y_{2}<0$ associated with the deviation from $\sigma_{x x}^{*}$. The starting point for the flow in Fig. 13 at microscopic length scales is given by the expressions for the conductivity tensor in the self-consistent Born approximation (SCBA) (Ando et al., 1975). 
While the field theory with the Lagrangian (5.11) provides a very appealing framework for the quantum Hall transition it has major shortcomings. Up to date it has not led to quantitative predictions, besides the existence of isolated critical fixed points. It has not been possible to calculate the longitudinal conductivity $\sigma_{x x}^{*}$ at the critical point, the localization length exponent $\nu$, or the irrelevant exponent $y_{2}$. The validity of the dilute instanton gas approximation that forms the basis of the approximate flow equations (5.12) has been questioned by Weidenmüller and Zirnbauer (1988). Recently, Zirnbauer (1994) has argued that the flow-diagram Fig. 13 does not describe the flow of the coupling constants in the nonlinear $\sigma$ model (5.11) and that a different theory is called for. We conclude that at present the most promising tool to obtain quantitative results about the critical properties of the integer quantum Hall system seems to be numerical simulation, on which we will now focus our attention.

\section{LOCALIZATION LENGTH}

The localization length $\xi(E)$ is one of the simplest quantities to calculate numerically that shows the occurrence of a metal-insulator transition. It can be defined by the asymptotic behavior of the modulus of the single-particle Green function

$$
G\left(\mathbf{r}, \mathbf{r}^{\prime} ; E\right)=\left\langle\mathbf{r}\left|\frac{1}{E-H}\right| \mathbf{r}^{\prime}\right\rangle .
$$

The disorder average of $G\left(\mathbf{r}, \mathbf{r}^{\prime} ; E\right)$ is a short-ranged function due to the average over the phase fluctuations. It decays on the scale of the elastic mean-free path and does not show critical behavior. By averaging the modulus these phase cancellations are avoided and $\overline{\left|G\left(\mathbf{r}, \mathbf{r}^{\prime} ; E\right)\right|}$ becomes long-ranged near the phase transition. $\left|G\left(\mathbf{r}, \mathbf{r}^{\prime} ; E\right)\right|$ is not a selfaveraging quantity and has a broad distribution. The inverse localization length

$$
\xi(E)^{-1}=-\lim _{\left|\mathbf{r}-\mathbf{r}^{\prime}\right| \rightarrow \infty} \frac{1}{\left|\mathbf{r}-\mathbf{r}^{\prime}\right|} \overline{\ln \left|G\left(\mathbf{r}, \mathbf{r}^{\prime} ; E\right)\right|}
$$

on the other hand is a self-averaging quantity so that it takes on the same value for almost all members of the disorder ensemble. The self-averaging property makes it particularly 
useful for numerical calculations. In Eq. (6.2) the energy $E$ has to have an imaginary part since after averaging $\overline{\left|G\left(\mathbf{r}, \mathbf{r}^{\prime} ; E\right)\right|}$ is non-analytic on the real axis. Using the self-averaging property and calculating the localization length for a single disorder realization in a finite system this imaginary part can be set to zero as long as $E$ is not an eigenenergy of the system.

In order to implement a finite-size scaling analysis of the localization length one studies long strips (in $d=2$ dimensions) or bars (in $d=3$ dimensions) and calculates the localization length along the infinite direction of the system. This localization length then depends on the perpendicular dimensions of the system (MacKinnon and Kramer, 1981; MacKinnon and Kramer, 1983). In section VIA we review a method to calculate recursively the localization length for systems of this geometry and extend it to the case of the random Landau matrix.

Another way to define the localization length is to identify it with the inverse of the smallest Lyapunov exponent of the transfer matrix of the system (MacKinnon and Kramer, 1981; MacKinnon and Kramer, 1983; Pichard and Sarma, 1981). We will return to this in section $\nabla T B$.

\section{A. Recursive Green Function Method}

The recursive Green function method was developed by MacKinnon and Kramer (1981; 1983) for the Anderson model (3.31). In one dimension the corresponding Schrödinger equation $(3.32)$ reads

$$
a_{i+1}=\left(E-\epsilon_{i}\right) a_{i}-a_{i-1}
$$

where the hopping matrix element was set to unity. Eq. (6.3) allows one to calculate recursively the wave function amplitudes $a_{i}$. In higher dimensions the corresponding equation is

$$
A_{i+1}=\left(E-H_{i}\right) A_{i}-A_{i-1} .
$$


$H_{i}$ is the Hamiltonian of the $i^{\text {th }}(d-1)$-dimensional slice of the $d$-dimensional "bar" and all quantities are $(d-1)$-dimensional matrices. Eq. (6.4) can be rewritten in terms of Green functions $G_{1, n}$ coupling sites in slice 1 to sites in slice $i$,

$$
\begin{aligned}
G_{1, i+1} & =G_{1, i} G_{i+1, i+1}, \\
G_{i+1, i+1} & =\left(E-H_{i+1}-G_{i, i}\right)^{-1} .
\end{aligned}
$$

Iterating Eq. 6.5) the matrix elements of the Green function connecting sites at both ends of long "bars" can be calculated without need for diagonalization of the Hamiltonian of the whole system.

Eq. (6.5) is tailored to the Anderson model with constant nearest-neighbor couplings. To apply this method to the Hamiltonian (3.16) it has to be modified. The random Landau matrix $\left\langle n k_{1}|V| n k_{2}\right\rangle$ of Eq. (A1) describes a random one-dimensional tight-binding Hamiltonian similar to the Anderson Hamiltonian but with long-ranged hopping matrix elements. To be able to apply recursion relations similar to Eqs. 6.5) Aoki and Ando (1985; 1985) reinterpreted the one-dimensional Hamiltonian $\left\langle n k_{1}|V| n k_{2}\right\rangle$ as the Hamiltonian of a twodimensional system with couplings only between sites in neighboring slices. This is possible since $\left|\left\langle n k_{1}|V| n k_{2}\right\rangle\right|^{2}$ is essentially zero for $\left|k_{1}-k_{2}\right|>K$ and can be neglected, where $K$ is a constant of order $\left(\beta l_{c}\right)^{-1}$ such that $N_{K}=K L_{y} / 2 \pi$ is an integer. States with $k \in[(i-1) K+1, i K[$ belong to slice $i$ and are coupled only to states in slices $i-1, i$, and $i+1 .\left\langle n k_{1}|V| n k_{2}\right\rangle$ can then be rewritten as a tri-diagonal matrix with diagonal elements $H_{i, i}$ being the $N_{K} \times N_{K}$ submatrices of $\left\langle n k_{1}|V| n k_{2}\right\rangle$ connecting states within slice $i$ and off-diagonal elements $H_{i, i+1}$ being the $N_{K} \times N_{K}$ submatrices connecting states within slice $i$ with those in slice $i+1$. The matrix elements in the off-diagonal matrices $H_{i, i+1}$ are now random numbers in contrast to the case of the two-dimensional Anderson model so that the recursion relation (6.5) has to be modified to

$$
\begin{aligned}
G_{1, i+1} & =G_{1, i} H_{i, i+1} G_{i+1, i+1}, \\
G_{i+1, i+1} & =\left(E-H_{i+1}-H_{i+1, i} G_{i, i} H_{i, i+1}\right)^{-1} .
\end{aligned}
$$


A different approach was introduced by Huckestein (1990) who generalized the onedimensional Green function method to the case of long-ranged hopping matrix elements. Consider the random Landau matrix $H^{(K)}$ of dimension $K$ describing a system containing states $k=2 \pi i / L_{y}, i=1, \ldots, K$. In the following, we will label the states by integers $k$ instead of quasi-momenta $2 \pi k / L_{y}$. Adding the state $K+1$ to the system leads to a Hamiltonian $H^{(K+1)}$ that can be decomposed into a block-diagonal part $H_{0}$ and the couplings $H^{\prime}$ of state $K+1$ to the rest of the system,

$$
\begin{aligned}
H^{(K+1)} & =\sum_{k, k^{\prime}=1}^{K+1}|k\rangle\left\langle k|V| k^{\prime}\right\rangle\left\langle k^{\prime}\right|, \\
& =H_{0}+H^{\prime}, \\
H_{0} & =H^{(K)}+|K\rangle\langle K|V| K\rangle\langle K|, \\
H^{\prime} & =\sum_{k=1}^{K}(|k\rangle\langle k|V| K+1\rangle\langle K+1|+\text { h.c. }),
\end{aligned}
$$

where we have suppressed the Landau level index. The related Green functions are $G^{(K)}=$ $\left(E-H^{(K)}\right)^{-1}$ and $G_{0}=\left(E-H_{0}\right)^{-1}$ for which we obtain the recursion relation

$$
\begin{aligned}
G_{0} & =G^{(K)}+\frac{|K+1\rangle\langle K+1|}{E-\langle K+1|V| K+1\rangle}, \\
G^{K+1} & =G_{0}+G_{0} H^{\prime} G^{K+1} .
\end{aligned}
$$

The last relation can be used to calculate $G^{K+1}$ owing to the fact that the matrix $H^{\prime}$ only couples to site $K+1$. Using the property that the random Landau matrix is a banded matrix the dimensions of the matrices in Eqs. (6.8) can be kept finite even in the limit $K \rightarrow \infty$. Choosing matrices of dimension $2 M / \beta$, where $M=L_{y} / \sqrt{2 \pi} l_{c}$, results in neglecting matrix elements of order $\exp (-4 \pi)$ compared to the diagonal matrix elements. Let $N$ be the dimension of these matrices, then the recursion relation (6.8) for the matrix elements are

$$
\begin{aligned}
& \left\langle K+1\left|G^{(K+1)}\right| K+1\right\rangle= \\
& \left(E-\langle K+1|V| K+1\rangle-\sum_{i, j=K-N+2}^{K}\langle K+1|V| i\rangle\left\langle i\left|G^{(K)}\right| j\right\rangle\langle j|V| K+1\rangle\right)^{-1}
\end{aligned}
$$

for the diagonal matrix element, 


$$
\left\langle i\left|G^{(K+1)}\right| K+1\right\rangle=\left\langle K+1\left|G^{(K+1)}\right| K+1\right\rangle \sum_{j=K-N+2}^{K}\left\langle i\left|G^{(K)}\right| j\right\rangle\langle j|V| K+1\rangle
$$

for $i \leq K$, and

$$
\left\langle i\left|G^{(K+1)}\right| j\right\rangle=\left\langle i\left|G^{(K)}\right| j\right\rangle+\sum_{k=K-N+2}^{K}\left\langle i\left|G^{(K)}\right| k\right\rangle\langle k|V| K+1\rangle\left\langle K+1\left|G^{(K+1)}\right| j\right\rangle
$$

for $i \leq K$ and $j \leq K$.

The localization length $\lambda_{M}$ of a system with width $M=L_{y} / \sqrt{2 \pi} l_{c}$ and length $K$ is given by

$$
\lambda_{M}^{-1}(E ; K)=-\frac{L_{y}}{K 2 \pi l_{c}^{2}} \ln \left|\left\langle 1\left|G^{(K)}(E)\right| K\right\rangle\right|
$$

In calculating $\left\langle 1\left|G^{(K)}\right| K\right\rangle$, we are faced with the difficulty that this is an exponentially decreasing quantity. To circumvent this problem we introduce variables $q_{K}=$ $\left\langle 1\left|G^{(K)}\right| K\right\rangle /\left\langle 1\left|G^{(K-1)}\right| K-1\right\rangle$ for the change in $\left\langle 1\left|G^{(K)}\right| K\right\rangle$ when the system size is increased by one state. In terms of the $q_{k}$, Eq. (6.12) is given by

$$
\begin{aligned}
\lambda_{M}^{-1}(E ; K) & =-\frac{M}{K \sqrt{2 \pi} l_{c}} \ln \left|\frac{\left\langle 1\left|G^{(K)}\right| K\right\rangle}{\left\langle 1\left|G^{(K-1)}\right| K-1\right\rangle} \frac{\left\langle 1\left|G^{(K-1)}\right| K-1\right\rangle}{\left\langle 1\left|G^{(K-2)}\right| K-2\right\rangle} \cdots\left\langle 1\left|G^{(1)}\right| 1\right\rangle\right| \\
& =-\frac{M}{K \sqrt{2 \pi} l_{c}} \sum_{i=1}^{K} \ln \left|q_{i}\right|,
\end{aligned}
$$

where we set $q_{1}=\left\langle 1\left|G^{(1)}\right| 1\right\rangle=(E-\langle 1|V| 1\rangle)^{-1}$. From Eq. (6.10) we get a recursion relation for $q_{K+1}$,

$$
q_{K+1}=\left\langle K+1\left|G^{(K+1)}\right| K+1\right\rangle \sum_{j=K-N+2}^{K}\left\langle 1\left|g^{(K)}\right| j\right\rangle\langle j|V| K+1\rangle,
$$

where $\left\langle 1\left|g^{(K)}\right| j\right\rangle=\left\langle 1\left|G^{(K)}\right| j\right\rangle /\left\langle 1\left|G^{(K)}\right| K\right\rangle$ is normalized to compensate for the exponential decay. With Eq. (6.11), we obtain the recursion relation for $\left\langle 1\left|g^{(K+1)}\right| j\right\rangle$,

$$
\begin{aligned}
& \left\langle 1\left|g^{(K+1)}\right| j\right\rangle=\frac{1}{q_{K+1}}\left(\left\langle 1\left|g^{(K)}\right| j\right\rangle\right. \\
& \left.+\sum_{k=K-N+2}^{K}\left\langle 1\left|g^{(K)}\right| k\right\rangle\langle k|V| K+1\rangle\left\langle K+1\left|G^{(K+1)}\right| j\right\rangle\right) .
\end{aligned}
$$




\section{B. Transfer-Matrix Method}

The transfer-matrix method deals with a physical situation where a long narrow disordered system is connected at both ends to semi-infinite ideal conductors. The transfer matrix $T$ relates the amplitudes on the right-hand side of the system to those on the left-hand side. For the Anderson model (6.4) the transfer matrix $T_{i}$ of slice $i$ is given by

$$
T_{i}=\left(\begin{array}{cc}
E-H_{i} & -I \\
I & 0
\end{array}\right),
$$

where $I$ is the unit matrix, since Eq. (6.4) can be rewritten as

$$
\left(\begin{array}{c}
A_{i+1} \\
A_{i}
\end{array}\right)=T_{i}\left(\begin{array}{c}
A_{i} \\
A_{i-1}
\end{array}\right) .
$$

For Chalker's and Coddington's network model of section IIID the transfer matrix $T$ consists of a product of four matrices, $T=A B C D$, where $A$ and $C$ are diagonal matrices with random phases, corresponding to the links connecting the nodes of the network, and $B$ and $D$ are block-diagonal, consisting of the $2 \times 2$ matrices $M$ of Eq. (3.51). Note that the network of Fig. 10 is periodic when using two columns of nodes as a unit.

From the definition of the transfer matrix it follows immediately that the transfer matrix of a long system is just the product of the transfer matrices of the slices composing the system,

$$
M_{n}=\prod_{i=1}^{n} T_{i},
$$

where $M_{n}$ is the transfer matrix of the system consisting of $n$ slices and $T_{i}$ is the transfer matrix of slice $i$. Oseledec's theorem (Oseledec, 1968) states that a limiting matrix $\Gamma$ exists,

$$
\Gamma=\lim _{n \rightarrow \infty}\left(M_{n}^{*} M_{n}\right)^{1 / 2 n}
$$

with eigenvalues $\exp \gamma_{1}, \ldots, \exp \gamma_{2 m}$, where $2 m$ is the dimension of the transfer matrix. The $\gamma_{i}$ are the characteristic Lyapunov exponents of $M_{n}$. The inverse localization length $\xi^{-1}$ is given by the Lyapunov exponent of smallest absolute magnitude, $\xi^{-1}=\min \left|\gamma_{i}\right|$. 


\section{OTHER SCALING QUANTITIES}

The localization length is by no means the only quantity that can be used in finite-size scaling studies. In fact, early numerical calculation on the QHE used the size-dependence of the Thouless number to obtain information about the critical behavior (Ando, 1983; Ando, 1984a; Ando, 1984b). Information about the localization properties of eigenstates can also be obtained from their topological character. Huo and Bhatt (1992) used the density of states with non-zero Chern number as a finite-size scaling quantity.

\section{A. Thouless Number}

Edwards and Thouless (1972) have argued that the sensitivity of eigenenergies of finite systems to changes in the boundary conditions can be used to distinguish between extended and localized states. For localized states the eigenenergies should be insensitive against the change in boundary conditions if the system size is large compared to the localization length. Extended states should feel the change in the boundary conditions and the shift in energy should be of the order of $\hbar / \tau$, where $\tau$ is the time it takes the electron to diffuse to the boundary (Thouless, 1974). In second-order perturbation theory the change in energy $\delta E$ when periodic boundary conditions are replaced by anti-periodic boundary conditions is approximately related to the conductivity $\sigma$ by (Licciardello and Thouless, 1975)

$$
\sigma L^{d-2}=\frac{e^{2}}{h} f \frac{\delta E}{\Delta E}=\frac{e^{2}}{h} f g(L)
$$

where $\Delta E$ is the mean level spacing at the Fermi energy and $f$ is a constant of order unity depending on details of the microscopic model. $g(L)$ is called the Thouless number. In the presence of a strong magnetic field Ando (1983) argued that the constant $f$ is $\pi / 2$. The Thouless number $g(L)$ for a particular level is a strongly fluctuating quantity as a function of level index. To get a less fluctuating quantity typically the geometric mean $\delta E=\exp \left(\left\langle\ln \delta E_{i}\right\rangle_{\text {ave }}\right)$ is used, where the average is taken over a given energy interval. On the other hand, the very fact that $g(L)$ is strongly fluctuating was used by Fastenrath, 
Janßen and Pook (1992) in a multifractal analysis of the integer quantum Hall transition (Sec. $\mathbb{X X C )}$.

\section{B. Chern Number}

While the Thouless number relates to the longitudinal conductivity $\sigma_{x x}$, the ability of an eigenstate to contribute to the Hall conductivity $\sigma_{x y}$ serves as another means of distinguishing extended from localized states. For a system with generalized periodic boundary conditions,

$$
t\left(\mathbf{L}_{j}\right)|\psi\rangle=\exp \left(i \theta_{j}\right)|\psi\rangle \quad(j=1,2)
$$

where $t\left(\mathbf{L}_{j}\right)$ is the magnetic translation operator (Zak, 1964; Haldane and Rezayi, 1985; Arovas et al., 1988), the Hall conductivity may be written as a sum over all occupied states below the Fermi energy E (Thouless et al., 1982; Pook and Hajdu, 1987)

$$
\begin{aligned}
\sigma_{x y}(E)= & \frac{e^{2}}{h} \sum_{m} \frac{i}{2 \pi} \int_{T^{*}} d^{2} \theta \Theta\left(E-E_{m}\right) \\
& \times\left(\left\langle\frac{\partial \tilde{\psi}_{m}(\theta)}{\partial \theta_{1}} \mid \frac{\partial \tilde{\psi}_{m}(\theta)}{\partial \theta_{2}}\right\rangle-\left\langle\frac{\partial \tilde{\psi}_{m}(\theta)}{\partial \theta_{2}} \mid \frac{\partial \tilde{\psi}_{m}(\theta)}{\partial \theta_{1}}\right\rangle\right), \\
\left|\tilde{\psi}_{m}\right\rangle= & \exp \left[-i\left(\theta_{1} x / L_{x}+\theta_{2} y / L_{y}\right)\right]\left|\psi_{m}\right\rangle,
\end{aligned}
$$

where $T^{*}$ is the torus $\theta_{i} \in[0,2 \pi[, i=1,2$. It has been shown by Thouless and co-workers (Thouless et al., 1982; Niu et al., 1985) and others (Avron et al., 1983; Kohmoto, 1985; Aoki and Ando, 1986; Pook and Hajdu, 1987) that the integral in Eq. (7.4) is $2 \pi$ times an integer $C_{1}(m)$, the first Chern index, which is a topological invariant characterizing the topological properties of the wave functions. Arovas et al. (1988) have shown that for states with finite $C_{1}(m)$ the zeros of the wave function can be moved to any position in real space by a suitable choice of the boundary angles $\theta_{1}, \theta_{2}$, while states with zero $C_{1}(m)$ have zeros that are confined in space. This leads to a natural distinction between extended and

localized wave function. Again, as in the discussion of the Thouless number, the sensitivity to boundary conditions is used to distinguish extended from localized states. Since the first 
Chern index is an integer it allows a clear definition of extended versus localized states even for finite systems.

\section{NUMERICAL CALCULATIONS}

First numerical calculations of the critical properties for two-dimensional systems in a strong magnetic field were performed by Aoki (1977; 1978; 1985), Ando (1983; 1984a; 1984b), and Schweitzer, Kramer, and MacKinnon (1984). Mostly due to the insufficient computing power available at the time these studies were not able to obtain accurate information about the behavior of the localization length or the Thouless number. Ando (1983; 1984a; 1984b) interpreted his results obtained from the size dependence of the Thouless number as being compatible with an extended state at a single energy in each Landau level, a result previously obtained by Ono (1982b) by summing a certain class of diagrams analytically. However, the divergence of the localization length obtained numerically was much weaker than Ono's result $\xi(E) \propto \exp \left(1 / E^{2}\right)$, where the energy is measured relative to the critical energy. While Ando was not able to extract a result for the localization length exponent $\nu$ from his data he found first signs of a single-parameter scaling law since his numerically constructed $\beta$ function for the conductance depended only the conductance itself. Schweitzer, Kramer, and MacKinnon (1984) on the other hand interpreted their results for the localization length as indicating a band of extended states separated from localized states in the band tails by two mobility edges.

In the following we will concentrate on more recent results that shed light on the critical behavior near the band centers of the Landau levels. In our discussion we have to keep in mind that numerical calculations always deal with finite systems while strictly speaking phase transitions exist only in infinite systems. Thus, we need a theoretical framework in which to analyze data for finite systems in order to extrapolate results to infinite system size. The finite-size scaling theory outlined in section $\mathbb{\square}$ provides such a framework.

We will first review calculations for Hamiltonians projected onto a single Landau level. 
In the strong magnetic field limit, $\hbar \omega_{c} \gg \Gamma$, this seems to be a reasonable approximation. The effects of Landau level mixing will be discussed in Sec. VIIID while the influence of a periodic potential on a single Landau level is considered in Sec. VIIIE.

\section{A. Lowest Landau Level}

The first calculation that produced reasonable estimates of the critical exponents for the quantum Hall system was performed by Aoki and Ando (1985)(Ando and Aoki, 1985). They used a real-space model for the disorder with the random potential given by a sum over either short-range scatterers (3.8) or Gaussian scatterers (3.11). Using the recursive Green function method of section VIA they calculated the localization length for strips of width 1.25 to $40.1 l_{c}$. In the direction perpendicular to the strip periodic boundary conditions were employed to avoid the effects of edge states extended along the edges of the strip. Aoki and Ando tried to interpret their data in terms of Pruisken's two-parameter scaling theory (Sec. VB). However, in view of later calculations (Huckestein and Kramer, 1989; Huckestein and Kramer, 1990) it has to be realized that their systems were too small to be in the critical region. Nevertheless, they found that the localization length diverges at a single energy at the center of the Landau band and estimated the localization-length exponent to be $\nu \approx 2$ in the lowest Landau level.

Huckestein and Kramer (1990) extended these calculations by introducing the random Landau-matrix model (Sec. [IIB). Comparing their results for the localization length with the results of Aoki and Ando shows (Huckestein and Kramer, 1989) that the different random potentials give the same results for a width of $40 l_{c}$, the largest calculated by Aoki and Ando, while for smaller widths differences are observed. Microscopic details of the disorder potential therefore seem to become irrelevant for the behavior of the localization length on this length scale for $\delta$-correlated potentials. In Fig. 14 the normalized localization length $\lambda_{M}(E) / M$ is plotted as a function of the system width $M=L_{y} /(2 \pi)^{1 / 2} l_{c}$ for energies between

0.01 and $1.0 \Gamma$ relative to the band center. The length of the systems was $2.5 \times 10^{5} l_{c}\left(2.5 \times 10^{4} l_{c}\right.$ 
for the widest systems) and the width ranged up to $321 l_{c}$. The data within the dotted region are compatible with a single-parameter scaling law

$$
\begin{aligned}
\lambda_{M}(E) & =M \Lambda(M / \xi(E)), \\
\xi(E) & =\xi_{0}\left|\frac{E-E_{c}}{\Gamma_{\sigma}}\right|^{-\nu} .
\end{aligned}
$$

At this point we want to elaborate on the significance of this statement. In Sec. VIA it was argued that the inverse localization length calculated from the Green function is self averaging for system length $L \rightarrow \infty$. Numerically, it can only be calculated for finite $L$. The value obtained fluctuates within the disorder ensemble. The statistical error can be estimated from sampling different realizations of the disorder. In addition, systematic errors of the order of $\xi(E) / L$ have to be expected. In the present calculation the statistical errors are typically less than $1 \%$, with possible systematic errors of the same order. Using a statistical test (Huckestein, 1990), it was determined that the data in the dotted region are compatible with both scaling relations in Eq. (8.1) within the statistical errors. Corrections to Eq. (8.1) larger than the statistical errors are ruled out by this procedure. The deviations from scaling behavior for smaller width in Fig. 14 are not only due to irrelevant scaling fields, as discussed in Sec. IVB, but are also associated with the particular one-dimensional limit of the system. As was pointed out by Ando and Aoki (1985) for $M \rightarrow 0$ the localization length can be calculated analytically. In this limit the distance $\Delta X=\Delta k l_{c}^{2}=2 \pi l_{c}^{2} / L_{y}$ between the center coordinates of neighboring Landau states becomes large compared to $l_{c}$ so that the matrix elements $\left\langle n k|V| n k^{\prime}\right\rangle$ fall off rapidly as a function of $\left|k-k^{\prime}\right|$. The random Landau matrix becomes a tri-diagonal Hermitean matrix corresponding to a tight-binding model with nearest-neighbor hopping. Applying the exact result for the localization length of that model (Thouless, 1974) we get

$$
\lim _{M \rightarrow 0} \frac{\lambda_{M}}{M}=\frac{2}{\pi} \frac{1}{\beta^{2}},
$$

independent of energy.

The critical region starts at system width $M=16$, the same size at which the differences between the real-space model of Aoki and Ando and the random Landau-matrix model 
vanish. For energies larger than $0.5 \Gamma$ no scaling is observed since the localization length does not decrease arbitrarily in the band tails but levels off at about one magnetic length. Fig. 15 shows the data from the dotted region of Fig. 14 scaled according to Eq. (8.1a). Note that this figure contains data not only from Fig. 14 but also from Figs. 17, 18 and from the network model (Sec. VIIIQ). The scaling relations Eq. (8.1) determine $\xi(E)$ only up to a constant prefactor. This prefactor can be fixed by observing that in the localized regime, $M / \xi \gg 1, \lambda_{M}(E)$ converges to $\xi(E)$ for large $M$ so that

$$
\lim _{x \rightarrow \infty} \Lambda(x)=x^{-1}
$$

The divergence of the localization length in Fig. 16 is given by the exponent

$$
\nu=2.35 \pm 0.03
$$

This value for the exponent is in remarkable agreement with the experimental values $2.3 \pm 0.1$ (Koch et al., 1991b) and 2.4 \pm 0.1 (Wei et al., 1994). By performing similar calculations for smaller systems of up to $150 l_{c}$ width Mieck (1990) obtained an exponent of $\nu=2.3 \pm 0.08$.

In the previous analysis the critical energy $E_{c}$ was taken to be the band center so that the localization length diverges only at a single energy. The absence of extended states within a finite range of energies is consistent with the field-theoretic picture of Sec. $V B$ and was proven for single Landau levels by Chalker (1987).

The influence of a correlation length $\sigma=l_{c}$ on the behavior of the localization length can be seen in Fig. 17 (Huckestein et al., 1992). Single-parameter scaling behavior is again observed for the data in the dotted region. The necessary width increases by a factor of 4 relative to the $\delta$-correlated potential. One reason for this behavior is the increase in the effective length scale by a factor $\beta^{2}=\left(l_{c}^{2}+\sigma^{2}\right) / l_{c}^{2}$. Furthermore, due to the one-dimensional limit Eq. (8.2) the flow in Fig. 17 starts at values of $\lambda_{M} / M$ smaller by a factor of $\beta^{2}$ compared to $\sigma=0$. For large systems, $M \geq 64$, the scaling behavior is the same as for $\sigma=0$. Consequently, the data can be fitted to the same scaling curve in Fig. 15 and the localization length exponent is again given by Eq. (8.4). The prefactor of the localization 
length is roughly independent of the correlation length if the correlation-length dependence of the bandwidth $\Gamma_{\sigma}=\Gamma_{0} / \beta$ (Ando and Uemura, 1974) is taken into account. This result is in contrast to calculations by Ando (1989b) that showed a strong dependence of the prefactor on the correlation length. Presumably, the difference can be attributed to the limited system size $M \leq 20$ of that calculation.

For significantly larger correlation length it seems difficult at present to reach the system width necessary to observe scaling behavior. For $\sigma=4 l_{c}$ Mieck (1990) estimated a value for $\nu$ of about 2.3 .

In another approach to the localization problem Huo and Bhatt (1992) calculated the Chern numbers of the eigenstates in finite systems using Eq. (7.4). By studying the size dependence of the disorder-averaged density of states with non-zero Chern numbers they found that a finite portion of the states in the band center is extended in the thermodynamic limit. Away from the band center they found that the width $\Delta E$ of the band of states with non-zero Chern number decreased with the area $A$ of the system as

$$
\Delta E \propto A^{-1 / 2 \nu}
$$

with $\nu=2.4 \pm 0.1$. Eq. (8.5) is obtained by assuming that the states are extended in an energy range where the localization length $\xi(E) \propto\left|E-E_{c}\right|^{-\nu}$ exceeds the linear system size $L$, and $A=L^{2}$. They obtain the same exponent from the scaling of average number $N_{c}$ of states with non-zero Chern number, $N_{c} \propto A^{y}$, with $y=1-1 / 2 \nu$.

Ando (1992) studied the transmission through a disordered system with edges connected to two ideal leads. Using a lattice model for the system he calculated the transmission probability through the system as a function of the width of the system. He found that the energy range $\Delta E$ over which the transmission probability rises from zero to unity scales with the width $M$ of the system as $\Delta E \propto M^{-1 / \nu}$ with $\nu=2.2 \pm 0.1$. In the edge-state picture the finite conductivity near the center of the Landau levels is due to backscattering between edge states located at opposite sides of the sample. The backscattering is facilitated by extended bulk states and hence scales with the bulk critical exponent $\nu$. 
Chalker and Daniell (1988) calculated numerically the two-particle spectral function $S(q, \omega ; E)$ near the critical energy $E_{c} . S\left(q, \omega ; E_{c}\right)$ is the Fourier transform of

$$
S\left(\mathbf{r}, \omega ; E_{c}\right)=\overline{\sum_{\alpha, \beta} \delta\left(E_{c}-\hbar \omega / 2-E_{\alpha}\right) \delta\left(E_{c}+\hbar \omega / 2-E_{\beta}\right) \psi_{\alpha}(\mathbf{0}) \psi_{\alpha}^{*}(\mathbf{r}) \psi_{\beta}(\mathbf{r}) \psi_{\beta}^{*}(\mathbf{0})}
$$

Employing current conservation $S(q, \omega ; E)$ can be expressed in terms of a wave-vector and frequency dependent diffusion coefficient $D(q, \omega)$,

$$
S(q, \omega ; E)=\frac{\rho(E) \hbar}{\pi} \frac{q^{2} D(q, \omega)}{\omega^{2}+\left(q^{2} D(q, \omega)\right)^{2}}
$$

For a scale-invariant system, i.e., at the critical energy $E_{c}$, the diffusion coefficient can only depend on the dimensionless quantity $q L_{\omega}$, where $L_{\omega}=\left(\hbar \rho\left(E_{c}\right) \omega\right)^{-1 / 2}$ is the size of a system with mean level spacing $\omega$. Comparing this result, which is supported by numerical calculations (Chalker and Daniell, 1988; Huckestein and Schweitzer, 1994), with the dynamic scaling relation (4.25) applied to the spectral function we can conclude that the dynamic critical exponent $z=2$.

In the limit $q, \omega \rightarrow 0$ the diffusion coefficient $D(q, \omega)$ becomes the diffusion constant $D_{0}$ that is related to the conductivity by the Einstein relation

$$
\sigma_{x x}^{c}=e^{2} \rho\left(E_{c}\right) D_{0}
$$

Chalker (1987) has shown that due to a sum rule the diffusion coefficient $D\left(q L_{\omega}\right)$ at large values of $q L_{\omega}$ is reduced,

$$
D\left(q L_{\omega}\right) \propto\left(q L_{\omega}\right)^{-\eta}
$$

A non-zero value for $\eta$ is related to the multifractal properties of eigenfunctions at the mobility edge discussed in Sec. [X. Chalker and Daniell obtain values of $\eta=0.38 \pm 0.04$ and $\sigma_{x x}^{c}=0.45 e^{2} / h$.

Huo, Hetzel and Bhatt (1993) improved the calculation of the conductivity at the critical point using the same method as Chalker and Coddington. They found a universal value of $\sigma_{x x}^{c}=0.5 e^{2} / h$ within uncertainties of about $10 \%$ for seven different kinds of disorder 
potentials. Using the Kubo formula (Aoki and Ando, 1981) they calculated the Hall conductivity to obtain $\sigma_{x y}^{*}=0.5 e^{2} / h$. While this result is trivial for random potentials, which are particle-hole symmetric on the average, they showed that it also holds for potentials with an asymmetric density of states, again with uncertainties of about $10 \%$.

\section{B. Higher Landau Levels}

While the behavior of the localization length in the lowest Landau level seems to be well described by a single-parameter scaling relation the situation in higher Landau levels seems to be less clear. Published values for the localization-length exponent $\nu$ in the second Landau level $n=1$ range from 2.3 (Mieck, 1990) to 6.2 (Mieck, 1993). Indeed, it has been argued that the critical exponent $\nu$ should be nonuniversal in higher Landau levels and should depend on details of the disorder potential, in particular the correlation length $\sigma$ of the disorder potential (Mieck, 1990; Mieck, 1993; Liu and Das Sarma, 1993; Liu and Das Sarma, 1994). Only recently have these discrepancies been reconciled with a universal value of $\nu$ by considering corrections to single-parameter scaling behavior (Huckestein, 1994).

The first estimate for the exponent $\nu$ in the second Landau level was again given by Aoki and Ando (1985) who found $\nu \approx 4$. The disorder potential consisted of short-range scatterers in this case. The first systematic study of the effect of the correlation length of the disorder was performed by Mieck (1990; 1993). He noticed that the correlations of the matrix elements Eq. (A1) in the random Landau model become independent of the Landau level index for large correlation length $\sigma$ (Huckestein, 1992). Mieck obtained values of $\nu \approx 6.2$ for $\sigma=0, \nu \approx 3.7$ for $\sigma=0.5 l_{c}$, and $\nu \approx 2.3$ for $\sigma=4 l_{c}$. In performing a fit to the single-parameter scaling relation (8.1) for the short correlation lengths he used data from the energy interval between $0.5 \Gamma_{\sigma}$ and $0.8 \Gamma_{\sigma}$.

Huckestein (1992) showed that for a correlation length $\sigma=l_{c}$ the scaling behavior of the system is indeed the same as in the lowest Landau level. Fig. 18 shows the normalized localization length. The data from the dotted region can be fitted to the scaling curve 
of Fig. 15. Note that Fig. 18 differs from Fig. 17 for the lowest Landau level only for small width. The behavior for short-ranged correlations with $\sigma=0$ as shown in Fig. 19 is drastically different from both the behavior in the lowest Landau level (Fig. 14) and the correlated potential (Fig. 18). The important features of Fig. 19 are the slow but noticeable decrease of $\lambda_{M} / M$ as a function of $M$ in the band center, the very weak energy dependence near the band center $(E<0.3 \Gamma)$, and the strong energy dependence towards the band tails $(E>0.3 \Gamma)$. The first observation shows that the system width is too small to reach the critical region of single-parameter scaling as $\lambda_{M}\left(E_{c}\right) / M=\Lambda_{c}$ is constant at the critical point. This prohibits an analysis of the data in terms of a single-parameter scaling relation. If the strong energy dependence in the tails of the band is still analyzed using a singleparameter scaling relation large, nonuniversal values for $\nu$ are obtained (Aoki and Ando, 1985; Mieck, 1990; Huckestein, 1992; Mieck, 1993; Liu and Das Sarma, 1993; Liu and Das Sarma, 1994). In view of the absence of scale invariance in the band center and the fact that these energies are mostly outside the critical energy range in the lowest Landau level it seems highly doubtful that these exponents describe the critical behavior of the system.

The weak system-size dependence in the band center is due to corrections to the singleparameter scaling laws as discussed in Sec. IVB. To analyze these corrections we have to connect the behavior in the band center for short correlation length to the universal single-parameter scaling at larger correlation length where the corrections are too small to be observed. From the dependence of $\lambda_{M}\left(E_{c}\right) / M$ on $\beta^{2}$ and $M$ in Fig. 20 we see that the asymptotic single-parameter scaling regime is reached at about $\beta^{2}=1.8$. The corrections for smaller values of $\beta^{2}$ show single-parameter scaling as a function of $\beta^{2}$ and $M$ as expected from two-parameter finite-size scaling with one relevant and one irrelevant scaling index (see Sec. IVB) (Huckestein, 1994). Eq. (4.24) for the localization length $\lambda_{M}$ reads

$$
\lambda_{M}\left(E_{c}, \beta^{2}\right)=M \Lambda\left(0, M^{-\left|y_{\mathrm{irr}}\right|} \zeta_{\mathrm{irr}}\right)
$$

where $\Lambda\left((M / \xi(E))^{1 / \nu}, 0\right)$ is the single-parameter scaling function of Eq. (8.1). The physical nature of the irrelevant scaling field $\zeta_{\text {irr }}$ is unclear, except that it depends in some way on 
the disorder potential, particularly on the correlation length parameter $\beta^{2}$. In the present context Eq. (8.10) is an ansatz that describes the numerical data well.

Relating the unknown irrelevant scaling field $\zeta_{\text {irr }}$ to an irrelevant length scale $\xi_{\text {irr }} \propto \zeta_{\text {irr }}^{1 /\left|y_{\text {irr }}\right|}$ we can rewrite Eq. (8.10) as

$$
\lambda_{M}\left(E_{c}, \beta^{2}\right)=M \Lambda\left(0,\left(M / \xi_{\mathrm{irr}}\right)^{-\left|y_{\mathrm{irr}}\right|}\right)
$$

This is a single-parameter scaling law similar to Eqs. (8.1) but this time describing the flow towards the critical point as indicated by the negative scaling index. By applying the same fitting procedure as in Sec. VIIIA we can obtain the scaling function $\Lambda(0, x)$ and the dependence of $\xi_{\text {irr }}$ on the correlation length of the disorder potential. Fig. 21 shows $\lambda_{M} / M$ as a function of $M / \xi_{\text {irr. }}$. The irrelevant length scale $\xi_{\text {irr }}$ increases by four orders of magnitude when the correlation length of the disorder potential is reduced from $0.8 l_{c}$ to 0 (Fig. 22). The overall scale of $\xi_{\text {irr }}$ is not given by the fitting procedure. It is approximately known by observing that is becomes of the order of the magnetic length $l_{c}$ as the correlation length approaches $l_{c}$. The asymptotic single-parameter scaling governed by the localization length $\xi$ can only be observed for length scales larger than the irrelevant length scale $\xi_{\text {irr }}$. Systems numerically accessible at present are 3 to 4 orders of magnitude too narrow to reach the asymptotic single-parameter scaling regime. The effects seen in all previous numerical calculations for short-ranged potentials in the second Landau level do not reflect the asymptotic single-parameter scaling regime but are dominated by corrections due to irrelevant scaling fields. The fact that these corrections can be analyzed using a twoparameter scaling relation shows that the system sizes accessible in numerical simulations are in fact sufficient to reach the critical regime. It is just the description in terms of single-parameter scaling laws that becomes valid only after the irrelevant length scale $\xi_{\text {irr }}$ is exceeded.

For small values of $M^{-\mid y_{\text {irr }}} \zeta_{\text {irr }}$ we can expand the function $\Lambda$ in Eq. (8.10) to get

$$
\Lambda=\Lambda_{c}+b M^{-\left|y_{\text {irr }}\right|} \zeta_{\text {irr }}+\cdots
$$


From the width dependence of $\Lambda-\Lambda_{c}$ in Fig. 23 we get the irrelevant scaling index $y_{\text {irr }}=$ $-0.38 \pm 0.04$ (Huckestein, 1994). For $M / \xi_{\text {irr }} \gtrsim 0.1$ this exponent describes the data well. This suggests that, at least in this regime, corrections due to further irrelevant scaling fields with scaling indices less than $y_{\text {irr }}$ are negligible.

The structure of the fixed point described by the two-parameter scaling relation

$$
\lambda_{M}\left(\Delta E, \zeta_{\text {irr }}\right)=M \Lambda\left(M^{1 / \nu} \Delta E, M^{-\left|y_{\text {irr }}\right|} \zeta_{\text {irr }}\right)
$$

is identical to the one expected by Pruisken (1987) for the delocalization fixed points at half-integer $\sigma_{x y}$ (see Fig. 13),

$$
\lambda_{M}\left(\Delta \sigma_{x y}, \Delta \sigma_{x x}\right)=M \Lambda\left(M^{1 / \nu} \Delta \sigma_{x y}, M^{-|\tilde{y}|} \Delta \sigma_{x x}\right) .
$$

Here $\Delta \sigma_{i j}$ are the linear deviations from the fixed-point values of $\sigma_{i j}$. Near the critical point $\Delta \sigma_{x y}$ is proportional to $\Delta E$ so that the scaling with respect to the first variables in Eqs. (8.13) and (8.14) is the same. For the second variables, $\zeta_{\text {irr }}$ and $\Delta \sigma_{x x}$ a direct relation is not obvious. Though both quantities depend on the correlation length of the potential it is not clear that $\zeta_{\text {irr }}$ is proportional to $\Delta \sigma_{x x}$.

We can understand the dependences of the corrections to single-parameter scaling on the correlation length of the disorder potential and the Landau level index in the field-theoretical framework. As discussed in Sec. $\mathrm{VB}$ the mean-field approximation to the field theory is given by the non-critical SCBA (Pruisken, 1987). To observe the localization-delocalization fixed point brought about by fluctuations around the mean-field saddle point the system size has to exceed the localization length in SCBA. The scaling in this approximation is described by the corresponding unitary non-linear $\sigma$-model without topological term and is given by the $\beta$ function in Eq. (5.10). The localization length is obtained by integrating the leading term in Eq. (5.10) for the unitary case. In terms of the conductivity $\sigma_{x x}^{0}$ on small length scales it is given by (Wei et al., 1985)

$$
\xi_{0}=l_{c} \exp \left(\pi^{2}{\sigma_{x x}^{0}}^{2}\right) .
$$


Using the result for the conductivity in SCBA for short-range scatterers (Ando and Uemura, 1974) the dependence on Landau level index $n$ is

$$
\xi_{0}(n)=l_{c} \exp \left[(2 n+1)^{2}\right]
$$

While this length scale is of the order of the magnetic length in the lowest Landau level it is more than 3 orders of magnitude larger for $n=1$. This explains why the corrections are not observed in the lowest Landau level even for $\delta$-correlated potentials. The strong variation of the irrelevant length scale in Fig. 22 can also be understood from the range dependence of the conductivity in SCBA and Eq. (8.15). For $n=1$ the conductivity decreases by about a factor of 6 between $\sigma=0$ and $\sigma=l_{c}$. The corresponding length scale $\xi_{0}$ decreases from about $10^{4} l_{c}$ to about one magnetic length. In the lowest Landau level the conductivity changes by a factor of 2 and $\xi_{0}$ decreases only by about the same factor.

Direct calculations of the critical value of the longitudinal conductivity $\sigma_{x x}$ are missing in higher Landau levels. Ando's results for the Thouless numbers for a lattice system with several Landau levels do not show a strong Landau level dependence as expected from SCBA (Ando, 1989a). It has been suggested that zero-temperature quantum phase transitions in two dimensions should have a universal value for the conductivity at the transition (Fisher et al., 1990; Fisher, 1990; Lee et al., 1992). Lee, Wang, and Kivelson (1993) argued that the critical value $\sigma_{x x}^{*}$ is related to the fixed-point scaling amplitude $\Lambda_{c}$. They obtain $\sigma_{x x}^{*}=1 / 2$ for $\Lambda_{c}=1 / \ln (1+\sqrt{2})$ which is very close to the best-fit result $\Lambda_{c}=1.14 \pm 0.02$ (Huckestein, 1994). Since the scaling curve Fig. 15, and in particular $\Lambda_{c}$, is independent of Landau level index, this would support the notion of a universal value of $\sigma_{x x}^{*}=1 / 2$ in accordance with the numerical calculations in the lowest Landau level (Huo et al., 1993). Landau level independence of the critical conductivity is also a feature of Khmel'nitskii's flow diagram Fig. 13. 


\section{Network model}

The first single-parameter scaling function for the QHE was obtained by Chalker and Coddington (1988). They studied the scaling behavior of the network model described in Sec. IIID using the transfer-matrix method of Sec. VIB. Their scaling function is not reproduced here as it is identical to Fig. 15. In fact, Fig. 15 was obtained by jointly fitting data from the network model and the random Landau-matrix model for $n=0, \sigma=0$, $n=1, \sigma=l_{c}$, and $n=1, \sigma=l_{c}$, to a single scaling function. The exponent $\nu$ was estimated by Chalker and Coddington to be about $2.5 \pm 0.5$. The large uncertainties are due to the non-linear dependence of the parameter $\theta$ on energy, Eq. (3.53). From the fact that the scaling functions of the models are identical it follows that the exponents are identical, too.

The network model has the remarkable feature that it shows very small finite-size corrections to single-parameter scaling. In contrast to the systems discussed above the data are close to the scaling function Fig. 15 already for small system width. This behavior can be understood from the different one-dimensional limits of the models. In this limit the normalized localization length of the network model is given by (Jaeger, 1991)

$$
\frac{\lambda_{M}(E)}{M}=\left(\ln 2+\ln \cosh \left(\frac{\pi \gamma}{2}\right)\right)^{-1}
$$

where $\gamma$ is related to the energy $E$ by Eq. (3.54). In contrast to Eq. (8.2) the network model retains an energy dependence in the one-dimensional limit and the value at the critical energy $\lambda_{M}\left(E_{c}\right) / M=1 / \ln 2$ is only about $20 \%$ larger than the fixed point value $\Lambda_{c}$.

A generalization of the network model in which the $\theta$ 's are taken to be random variables was discussed by Chalker and Eastmond (1993) and Lee, Wang, and Kivelson (1993). From finite-size scaling studies they find that this generalization is an irrelevant perturbation of the original model. If the distribution of the $\theta$ 's is narrow the scaling behavior is unchanged. Lee, Wang, and Kivelson obtain $\nu=2.43 \pm 0.18$ in this case. In the limit that the width of the distribution of $\theta$ 's goes to infinity the system behaves classically since each saddle point transmits only into one of the two possible links. Lee, Wang, and Kivelson find singleparameter scaling with $\nu=1.29 \pm 0.05$ compatible with the classical percolation exponent 
4/3. The classical percolation fixed point is unstable against quantum effects and the system flows towards the quantum fixed point described by $\nu=2.35 \pm 0.03$. Close to this fixed point the corrections to single-parameter scaling due to the finite width of the distribution of $\theta$ 's can be analyzed in the same way as discussed above. It was in this context that Chalker and Eastmond (1993) pioneered the analysis in terms of irrelevant scaling fields in the context of the QHE. They obtained the irrelevant scaling index $y_{\text {irr }}=-0.38 \pm 0.02$ in agreement with the results in the previous section. However, for the network model the corrections to the fixed-point value $\Lambda_{c}$ are negative as opposed to Fig. 21 where they are positive.

\section{Effects of Landau Level Coupling and Spin-Orbit Interaction}

When the strength of the disorder is not negligible compared to the cyclotron energy $\hbar \omega_{c}$ one has to go beyond the single-Landau-level approximation and include the effect of Landau level coupling by the disorder potential. In this context two different questions are important. For weak Landau level coupling such that each Landau level still contains a critical energy, does the Landau level coupling change the critical behavior at these critical energies? On the other hand, for strong disorder the system is expected to become insulating eventually (cf. Sec. VA . In this limit the question is: How do the divergences in the localization length disappear with increasing disorder? Numerically these question are hard to answer since the inclusion of several Landau levels necessitates much smaller systems so that finite-size effects become very prominent. It is also not obvious exactly how many Landau levels have to be taken into account to capture the essential physics.

The first question has been approached by Liu and Das Sarma (1994). They considered the two lowest Landau levels and studied the scaling behavior of the localization length as a function of the strength of the Landau level coupling for short correlation length of the potential. They find that while in the lowest Landau level the critical exponent $\nu$ does not change when the Landau level coupling is included and is close to the value of Eq. (8.4), in the $n=1$ Landau level there is a change in the exponent. While Liu and Das Sarma erroneously 
concluded from these data that Landau level coupling is essential for universal scaling for short correlation length, we can understand this behavior by considering the influence of Landau level coupling on the magnitude of corrections to scaling. With increasing Landau level coupling the inter-Landau level scattering increases and the conductivity on short length scales decreases. From Eq. 3.15) we see that this drastically decreases the characteristic length scale for single-parameter scaling. The system thus moves closer to the quantum critical point and finite-size corrections become smaller.

The dependence of the critical energies on Landau level mixing has been studied by Ando, both for continuous systems (1984b) and for lattice systems (1989a), using the Thouless number. For $\delta$-function scatterers and a Hamiltonian projected onto the three lowest Landau levels he finds that the localization length diverges at critical energies in each Landau level and that these energies move upward in energy when the Landau level coupling is increased. It also has to be pointed out that when the level broadening becomes comparable to the Landau level separation the localization length becomes very large, even for energies between the original Landau levels. For lattice systems the critical energies also move upward and eventually vanish. The square-lattice system studied by Ando is symmetric with respect to the center of the tight-binding band and for every Landau level below this center there exists another Landau level above with the opposite Hall conductivity. When two Landau levels merge in the center of the tight-binding band their contribution to the Hall conductivity vanishes and so does the associated critical energy. Due to this symmetry for the lattice system it is not clear whether the upward motion of the critical energy with increasing Landau level coupling is a generic feature.

The influence of spin degeneracy on the critical behavior has been studied by Lee and Chalker (1994) and Wang, Lee, and Wen (1994b) in an extension of the network model. To account for the spin degree of freedom two quantum-mechanical fluxes are carried by each link. The random phases on the links are then replaced by random $U(2)$ matrices. The numerical results of both calculations support the existence of two separate plateau transitions even in the spin-degenerate case. The critical exponent of each transition is 
in agreement with Eq. (8.4) for single Landau levels. If, on the other hand, the data are analyzed assuming a single critical energy over a limited range in energy a fit with an exponent $\nu \approx 5.8$ is possible.

\section{E. Presence of a Periodic Potential}

In this section we want to consider the influence of an additional periodic potential on the scaling behavior of the integer QHE. For definiteness, let us consider a periodic potential of the form

$$
V(\mathbf{r})=4 E_{0} \cos (\sqrt{2} \pi x / a) \cos (\sqrt{2} \pi y / a)
$$

In the absence of disorder each Landau level splits into $p$ subbands if $\alpha=2 \pi l_{c}^{2} / a^{2}=q / p(\mathrm{Az}-$ bel', 1964; Hofstadter, 1976) and the Hall conductivity in the gaps between these subbands is quantized in integer multiples of $e^{2} / h$ (Thouless et al., 1982). While the contribution of each subband to the Hall conductivity takes on large positive and negative values if $p$ is large, the sum of these contributions over a whole Landau level equals unity. We see that at least in the absence of disorder the additional periodic potential is quite relevant in that it completely changes the phase diagram and produces phases with new quantized values of the Hall conductivity. Under the influence of disorder the subband gaps will eventually be filled in and the intricate structure of the phase diagram will evolve into the simple phase diagram of the integer QHE Fig. 13.

The phase diagram in Fig. 24 was obtained by performing finite-size scaling studies for a system with $\alpha=3 / 5$ as a function of the ratio $\Gamma / E_{0}$ of the strength of disorder to periodic potential (Huckestein, 1993). The Hall conductivity was calculated from the Diophantine equation

$$
p \sigma+q s=t
$$

where $\sigma$ is the Hall conductivity in units of $e^{2} / h, t$ labels the subband gap, and $s$ is an integer (Thouless et al., 1982). In the presence of disorder the Hall conductivity can only 
change when a critical energy moves through the Fermi energy. For strong disorder there is only one critical energy at the center of the Landau level where the Hall conductivity changes from zero to one, while for weak disorder all phases of the non-disordered system are recovered.

The preceding discussion raises the question whether or not a periodic potential is a relevant perturbation of the fixed point at the center of the Landau level. For fixed system width the ratio $\lambda_{M}\left(E_{c}\right) / M$ increases with increasing strength of the periodic potential and decreases with increasing system width for fixed $E_{0}$ (Huckestein, 1994). The analysis of this behavior shows that it is compatible with the scaling due to an irrelevant scaling field according to Eq. (8.11). The scaling index obtained in this way is within error bars the same as in the second Landau level and in the network model, $y_{\text {irr }}=-0.38 \pm 0.04$. Thus the periodic potential is irrelevant at the fixed point at the center of the Landau level. The universality of the irrelevant scaling index suggests that further scaling indices are sufficiently small compared to $y_{\text {irr }}$ so that they are not observed in the numerical simulations.

The critical behavior at the other fixed points introduced by the periodic potential is still an open question. Numerically it is a hard problem since the finite-size corrections to single-parameter scaling become very strong and the position of the critical energy is not known a priori as it is for the critical state at the center of the Landau level. Since the new fixed points are connected to the band-center fixed point (see Fig. 24) one could conjecture that the critical behavior is the same for all fixed points.

\section{MULTIFRACTAL ANALYSIS}

So far our discussion focused on quantities, like the localization length, that are selfaveraging in the thermodynamic limit, even at the localization-delocalization transition. These quantities are a rather special class. In general, phase transitions are accompanied by

\footnotetext{
${ }^{3}$ At least for certain values of $\alpha$.
} 
large fluctuations in physical observables. At the transition where the natural length scale, the localization length, diverges these fluctuations appear on all length scales. In this section we want to analyze critical fluctuations of local observables in terms of multifractal measures. We will first outline the language of multifractal analysis, then apply this apparatus to computer simulations, and finally put this discussion into the context of the scaling theory.

\section{A. Generalized Dimensions and Singularity Strengths}

The multifractal analysis of measures was pioneered by Mandelbrot (1983) and further developed by, among others, Hentschel and Procaccia (1983), Halsey et al. (1986), and Chhabra and Jensen (1989). A recent review in the context of the localization-delocalization transition was written by Janßen (1994).

Consider a normalized measure on a two-dimensional square of linear dimension $L$,

$$
\int_{L^{2}} d \mu=1
$$

An example of such a measure is the local density, i.e., the squared modulus $|\psi(\mathbf{r})|^{2}$ of an eigenfunction of the Hamiltonian. The large fluctuations of this quantity can be seen in Fig. 25 where the local density for a state at the center of the lowest Landau level is shown.

With this measure we can define box-probabilities $p(l)$ by integrating the measure over squares $\Omega(l)$ of linear dimension $l$,

$$
p(l)=\int_{\Omega(l)} d \mu=\int_{\Omega(l)} d^{2} r|\psi(\mathbf{r})|^{2} .
$$

In our example $p(l)$ is just the probability to find an electron in the box $\Omega(l)$. The dimension of the support of the wavefunction is $d=2$, since the wavefunction has only isolated zeros. By covering the system with squares $\Omega(l)$ we get $N(\lambda)=\lambda^{-d}$ different box-probabilities $p_{i}(l)$, where $\lambda=l / L$. Due to the normalization $\sum_{i=1}^{N(\lambda)} p_{i}(l)=1$ the average box-probability scales with the ratio $\lambda$ as

$$
\langle p(l)\rangle_{L} \propto \lambda^{d}
$$


where the average is defined as

$$
\langle A\rangle_{L}=\frac{1}{N(\lambda)} \sum_{i=1}^{N(\lambda)} A_{i}
$$

The scaling law (9.3) is not useful in distinguishing between localized, extended, and critical wavefunctions as it holds for all normalized wavefunctions. This situation changes if we look at the scaling of moments of the box-probabilities. In the absence of length scales these will also show power law scaling but with nontrivial exponents $\tau(q)$,

$$
\left\langle p^{q}(l)\right\rangle_{L} \propto \lambda^{d+\tau(q)} .
$$

Here $q$ is real, but not necessarily integer. For a homogeneous measure, $p_{i}(l)=p(l)=$ $1 / N(\lambda)$, one finds $\tau(q)=(q-1) d$. We therefore define generalized dimensions $D(q)$, such that

$$
\tau(q)=(q-1) D(q) .
$$

We need to clarify under which conditions we expect power law scaling as in Eq. (9.5) to hold. As already pointed out, the localization length $\xi$ must not set a relevant length scale. For finite system size $L$ this gives the condition $L \ll \xi$. For short length scales microscopic lengths become important. In the quantum Hall system wavefunctions are smooth on scales of the magnetic length or the correlation length of the disorder. In order to see power law scaling we therefore need $l_{m} \ll l$, where $l_{m}$ is a microscopic length. In summary, the condition for power law scaling is

$$
l_{m} \ll l<L \ll \xi .
$$

In the limit $L \rightarrow \infty$ this condition is fulfilled only at the critical energy where the localization length diverges. In this limit the generalized dimensions are given by

$$
(q-1) D(q)=\lim _{\lambda \rightarrow 0} \frac{\ln \left\langle p^{q}(\lambda)\right\rangle_{L}}{\ln \lambda}-d .
$$

For finite system size the condition (9.7) is met over a finite range of energies. In this case the generalized dimensions can be estimated from the slope of $\ln \left\langle p^{q}(\lambda)\right\rangle_{L}$ vs. $\ln \lambda$ over a range of parameters that satisfies Eq. (9.7). 
For $q=0$ we find from Eq. (9.5) that $D(0)=d$ is the dimension of the support of the measure. The exponent $D(2)$ is related to the scaling exponent $d^{*}$ of the inverse participation ratio, defined by Wegner (1980)

$$
P^{(2)}=\int d^{2} r|\psi(\mathbf{r})|^{4} \propto L^{-d^{*}} .
$$

Formally, this equation is obtained from Eq. (9.5) for $q=2$ in the limit $l \rightarrow 0$. In this limit $d^{*}=D(2)$. In the limit $q \rightarrow 1, p^{(q-1)}$ can be expanded in Eq. (9.8) and $D_{1}$ is given by

$$
D(1)=\lim _{\lambda \rightarrow 0} \frac{\langle p(\lambda) \ln p(\lambda)\rangle_{L}}{\langle p(\lambda)\rangle_{L} \ln \lambda} .
$$

$D(1)$ is sometimes called the information dimension.

The function $D(q)$ has the following analytic properties that we will only sketch here. For a complete discussion see (Hentschel and Procaccia, 1983; Halsey et al., 1986). D(q) is a monotonously decreasing, positive function of $q$ bounded from above and below by $D_{-\infty}=D(-\infty)$ and $D_{\infty}=D(\infty)$, respectively, the generalized dimensions that describe the scaling of the minima and maxima of the measure.

Another way of expressing the multifractal properties of a measure is the spectrum of singularity strengths $f(\alpha)$. It is related to the function $\tau(q)$ by a Legendre transform, i.e., we change from the variable $q$ to the variable

$$
\alpha(q)=\frac{d \tau(q)}{d q} .
$$

$f(\alpha(q))$ is then implicitly given by

$$
f(\alpha(q))=\alpha(q) q-\tau(q) .
$$

From the analytical properties of the Legendre transform and the function $\tau(q)$ one finds (Chhabra and Jensen, 1989) that $f(\alpha)$ is a positive function of negative curvature with a single maximum. $\alpha$ ranges from $D_{\infty}$ to $D_{-\infty}$. At these points $f(\alpha)$ vanishes with infinite slope. For $q=1, f(\alpha)$ has slope 1 and $f(\alpha(1))=\alpha(1)=D(1)$. It takes on its maximum value $f\left(\alpha_{0}\right)=d$ for $q=0$, where $\alpha_{0}=\alpha(0)>d$. 
Chhabra and Jensen (1989) introduced a method to calculate $f(\alpha)$ directly. It has the advantage to be numerically more stable than calculating $\tau(q)$ and performing the Legendre transform (9.12). In addition to the box-probabilities we need to define a one-parameter family of normalized probabilities

$$
\mu_{i}(q, l)=\left[p_{i}(l)\right]^{q} / \sum_{i}\left[p_{i}(l)\right]^{q}
$$

that act as $q$-microscopes on the original measure. For each value of $q$ the corresponding values of $\alpha(q)$ and $\tilde{f}(q)=f(\alpha(q))$ can be calculated from

$$
\alpha(q)=\frac{\sum_{i} \mu_{i} \ln p_{i}}{\ln \lambda}
$$

and

$$
\tilde{f}(q)=\frac{\sum_{i} \mu_{i} \ln \mu_{i}}{\ln \lambda}
$$

Geometrically, the $f(\alpha)$ has a quite intuitive interpretation. If a certain box-probability $p_{i}(l)$ scales with a singularity strength $\alpha$, i.e., $p_{i}(l) \propto l^{\alpha}$, then $f(\alpha)$ is the fractal dimension of the set of boxes with singularity strength between $\alpha$ and $\alpha+d \alpha$.

Another aspect of the $f(\alpha)$ spectrum becomes clear when the average (9.4) is interpreted as an average over an ensemble instead of an average over the whole measure (Pook and Janßen, 1991; Fastenrath et al., 1992; Janßen, 1994). In terms of the distribution function $P(p, \lambda)$ the average of a function $A(p)$ can be written as

$$
\langle A(p, \lambda)\rangle=\int_{0}^{1} d p P(p, \lambda) A(p)
$$

Changing the integration variable to $\alpha=\ln p / \ln \lambda$ and defining $\tilde{P}(\alpha, \lambda) d \alpha=P(p, \lambda) d p$, Eq. (9.16) becomes

$$
\langle A(p, \lambda)\rangle=\int_{0}^{\infty} d \alpha \tilde{P}(\alpha, \lambda) P\left(\lambda^{\alpha}\right)
$$

From this we recover Eq. (9.5) for the scaling of the moments of $p$ if (Pook and Janßen, 1991) 


$$
\tilde{P}(\alpha, \lambda) \propto \lambda^{-f(\alpha)+d},
$$

where $f(\alpha)$ is given by Eq. (9.12). We see that $f(\alpha)$ describes the scaling of the whole distribution of box-probabilities, while the $\tau(q)$ describe the scaling of certain moments of that distribution.

The connection between the shape of the $f(\alpha)$ curve and the distribution function $\tilde{P}(\alpha, \lambda)$ becomes more transparent if we approximate the maximum of the $f(\alpha)$ spectrum by a parabola,

$$
f(\alpha)=d-\frac{\left(\alpha-\alpha_{0}\right)^{2}}{4\left(\alpha_{0}-d\right)} .
$$

Note that this parabolic approximation depends only on one parameter $\alpha_{0}$, besides the dimension $d$ of the support of the measure, which follows from the property $f(\alpha(1))=\alpha(1)$. The corresponding probability distribution of the box-probabilities is log-normal,

$$
\tilde{P}(\ln P / \ln \lambda, \lambda) \propto \exp \left(\frac{\left(\ln P-\alpha_{0} \ln \lambda\right)^{2}}{4\left(\alpha_{0}-d\right) \ln \lambda}\right) .
$$

We see that the absence of length scales that is reflected in the $f(\alpha)$ spectrum corresponds to broad distributions of local quantities like the box-probabilities. The parameter $\alpha_{0}$ that defines the parabolic approximation is the value of $\alpha$ at the maximum of $f(\alpha)$ and describes the scaling of the typical value $p_{\mathrm{typ}}(\lambda)$ of the box-probability defined by

$$
p_{\text {typ }}(\lambda)=\exp (\langle\ln p(\lambda)\rangle) \propto \lambda^{\alpha_{0}} .
$$

The multifractal density fluctuations lead to anomalous diffusive behavior (Chalker and Daniell, 1988). This is reflected in power-law decay of density correlations and slow decay of temporal wave-packet autocorrelations (Huckestein and Schweitzer, 1994). The exponent $\eta$ of density correlations,

$$
\overline{|\psi(\mathbf{r}) \psi(\mathbf{r}+\mathbf{R})|^{2}} \propto|\mathbf{R}|^{-\eta},
$$

is related to the generalized dimension $D(2)$. If we replace in Eq. (9.4) the average by an ensemble average and express $\overline{p^{2}(l)}$ as an integral over (9.22), we get 


$$
\overline{p^{2}(l)} \propto l^{2 d-\eta}
$$

Comparing with Eqs. (9.5) and (9.6) yields the result

$$
\eta=d-D(2)
$$

At finite wave vector and frequency the exponent $\eta$ describes the decay of the wave-vector and frequency-dependent diffusion coefficient (Eq. (8.9)). The value $\eta=0.38 \pm 0.04$ obtained by Chalker and Daniell (1988) from this quantity agrees well with Eq. (9.24) and $D(2)=$ $1.62 \pm 0.02$ obtained by Huckestein and Schweitzer (1994).

The anomalous diffusive behavior can directly be seen in the spreading of wave packets (Chalker and Daniell, 1988; Huckestein and Schweitzer, 1994). While the variance $R(2, t)=$ $\int d^{2} r|\mathbf{r}|^{2}|\phi(\mathbf{r}, t)|^{2}$ of a wave packet $\phi(\mathbf{r}, t)$ at the critical point grows linearly in time, which merely reflects the scale invariance of the system, the return probability $p(t)=|\phi(\mathbf{0}, t)|^{2}$ to the origin is enhanced and scales like

$$
p(t) \propto \frac{1}{t^{1-\eta / 2}}=\frac{1}{t^{D(2) / 2}} .
$$

A nonzero value of $\eta$ should be observable in measurements of the Coulomb drag in double layer systems (Shimshoni and Sondhi, 1994) and in hot-electron relaxation rates and electron-electron and electron-phonon scattering rates (Brandes et al., 1994).

The scaling of the two-particle spectral function $S(q, \omega)$ with $q^{2} / \omega$ allows to relate the correlation dimension $D(2)$ of the local density to the correlation dimension $\tilde{D}(2)$ of the spectral measure associated with the local density (Ketzmerick et al., 1992; Huckestein and Schweitzer, 1994). In two dimensions, $\tilde{D}(2)=D(2) / 2$.

\section{B. Universality of Multifractal Spectra}

In this section we review some numerical results about $f(\alpha)$ spectra for different models of the quantum Hall system and different physical quantities. We start with our prototype measure, the local density $|\psi(\mathbf{r})|^{2}$. The $f(\alpha)$ spectrum in Fig. 26 is calculated for the density 
shown in Fig. 25. In addition to the numerical data the parabolic approximation (9.19) is shown with $\alpha_{0}=2.29 \pm 0.02$ (Huckestein et al., 1992). The variation of $\alpha_{0}$ for different eigenstates in the energy range where $\xi(E) \gg L$ is of the same order. This shows that the $f(\alpha)$ spectrum not only describes a single eigenstate but is a universal feature of the system studied. While these calculations were performed for a tight-binding Hamiltonian, the results agree with calculations by Pook and Janßen (1991) for a real space potential on a square of linear size $70 l_{c}$ who obtained $\alpha_{0}=2.3 \pm 0.07, D_{\infty}=0.95 \pm 0.1$, and $D_{-\infty}=3.7 \pm 0.1$ (Janßen, 1994).

These calculations give a strong indication that the $f(\alpha)$ spectrum of the local density is universal at the critical point and that exponents, such as $\alpha_{0}$, are suited to describe the phase transition. It is not clear to what extend this universality holds for other physical observables. Calculations by Huckestein and Schweitzer (1992a) for the equilibrium current density $|\mathbf{j}(\mathbf{r})|$ as the local observable (see Fig. 27), by Schweitzer (1992) for the local magnetization $|\mathbf{m}(\mathbf{r})|=|\mathbf{r} \times \mathbf{j}|$, and by Fastenrath, Janßen, and Pook (1992) for the Thouless numbers give the same spectrum within the error bars and support the notion of universality. On the other hand, it is easy to construct multifractals with different $f(\alpha)$ spectra. Given a normalized multifractal measure $p$ the normalized moments $p^{m}$ generate new measures with $\alpha^{\left[p^{m}\right]}(q)=m \alpha(m q)-\tau(m)$ and $f^{\left[p^{m}\right]}\left(\alpha^{\left[p^{m}\right]}\right)=f(\alpha(q m))$ (Janßen, 1994). While this $f(\alpha)$ spectrum is simply related to the spectrum of $p$ it is not identical.

\section{Multifractality and Scaling}

We now want to relate the results about the multifractal properties at the quantum Hall transition to the discussion about scaling in the previous sections.

Bauer, Chang, and Skinner (1990) considered the scaling of generalized inverse participation ratios [cf. Eq. (9.9)]

$$
P^{(q)}=\int d^{2} r|\psi(\mathbf{r})|^{2 q}
$$


as a function of system size $L$ and distance from the critical energy $\Delta E$. Wegner (1980) introduced critical exponents $\pi(q)$ defined by

$$
P^{(q)} \propto \Delta E^{\pi(q)}
$$

Using a standard finite-size scaling ansatz for $P^{(q)}$,

$$
P^{(q)}(L, E) \propto L^{-\tau(q)} F_{q}\left(L^{1 / \nu} \Delta E\right),
$$

the condition that $P^{(q)}$ is finite for $E \neq E_{c}$ leads to the scaling relation

$$
\pi(q)=\nu \tau(q) .
$$

In particular, for the inverse participation ratio one finds $\pi(2)=\nu D(2)$. Hikami (1986) calculated the exponent $\pi(2)=3.8 \pm 0.4$ for the lowest Landau level. Neglecting the multifractal structure of the wavefunctions and assuming $P^{(2)} \propto \xi^{-2}$ he concluded $\nu=\pi(2) / 2=1.9 \pm 0.2$. Using $D(2)=1.62 \pm 0.02$ obtained from numerical calculations (Huckestein and Schweitzer, 1994) and the correct relation (9.29), we get

$$
\nu=2.4 \pm 0.3,
$$

in agreement with the result (8.4) obtained from finite-size scaling of the localization length.

In the spirit of Shapiro (1987) one can try to study the scaling of the whole distribution of box-probabilities $P(p, \lambda)$. At the critical point this distribution becomes universal and is determined by the $f(\alpha)$ spectrum. The bulk of the distribution is log-normal with deviations in the tails of the distribution that account for the differences between the parabolic approximation and the true $f(\alpha)$ curve in Fig. 26. In this limit the parameter $\alpha_{0}$ characterizes the whole distribution. Away from the critical energy, as long as $\xi(E) \gg L$, the distribution will still show power-law scaling (9.18). Since the system moves towards the localized regime the typical box-probability $p_{\text {typ }}$ will decrease and by Eq. (9.21) $\alpha_{0}$ will increase. If single-parameter scaling of the distribution holds, $\alpha_{0}$ has to obey a finite-size scaling law,

$$
\alpha_{0}(E)=A\left(L^{1 / \nu} \Delta E\right)=\alpha_{0}\left(E_{c}\right)+a L^{1 / \nu}|\Delta E|+\cdots .
$$


The linear energy dependence is compatible with the numerical simulations presented in Fig. 28 (Huckestein and Schweitzer, 1992b), but these calculations do not rule out a quadratic energy dependence. For larger distances from the critical energy, when $\xi(E)$ becomes smaller than the system size $L$, power-law scaling breaks down on length scales larger than $\xi(E)$. Eq. (9.18) no longer describes the whole distribution $P(p, \lambda)$ and $\alpha_{0}$ loses its meaning as the single parameter describing the distribution.

Employing conformal invariance in two-dimensional systems Cardy (1984) was able to show that the scaling amplitude $\Lambda_{c}^{-1}=\lim _{M \rightarrow \infty} M / \lambda_{M}$ of the inverse correlation length $\lambda_{M}^{-1}$ of the correlation function of an operator on a cylinder is related to the scaling dimension $x$ of the operator in the plane by

$$
\Lambda_{c}^{-1}=2 \pi x
$$

For the local density one is tempted to identify $\Lambda_{c}$ with the amplitude derived from the localization length on the cylinder and $x$ with the exponent $\eta / 2$. This argument neglects the broad distribution of the local density and the corresponding broad distribution of the correlation functions. Instead of mapping a single correlation function from the plane onto the cylinder, in the presence of broad distributions one needs to map the whole distribution function. Since the scaling amplitude $\Lambda_{c}$ was extracted from averaging the logarithm of the density correlation function the corresponding scaling dimension describes the scaling of the typical correlations $x=\alpha_{0}-d$ (Janßen, 1994). Noting that, by convention, the localization length is defined by the absolute value of the Green function, we get

$$
\Lambda_{c}=\frac{1}{\pi\left(\alpha_{0}-d\right)}
$$

which yields $\Lambda_{c}=1.1 \pm 0.1$ in accordance with the finite-size scaling result. A similar relation has been derived by Ludwig (1990) for a random 2D ferromagnet.

The discussion of multifractals can be extended to the description of non-normalized observables $Q$ (Ludwig, 1990; Duplantier and Ludwig, 1991; Pook and Janßen, 1991; Janßen, 1994). If the normalized observables $p^{[Q]}=Q / \sum_{i}^{N} Q$ form a multifractal measure the 
distribution of $Q$ can be described by the $f^{[Q]}\left(\alpha^{[Q]}\right)$ spectrum of $p^{[Q]}$ and the normalization exponent $X^{[Q]}$, defined by

$$
\sum_{i}^{N} Q(l) \propto \lambda^{X^{[Q]}} .
$$

Analogous to Eq. (9.21) the scaling of the typical value $Q_{\text {typ }}$ is then given by (Janßen, 1994)

$$
Q_{\mathrm{typ}}(l)=\exp (\ln \langle Q(l)\rangle) \propto l^{\alpha_{0}^{[Q]}+X^{[Q]}}
$$

Janßen (1994) interpreted $Q_{\text {typ }}(l)$ as the value of $Q$ for a system of size $l$ and the average in Eq. (9.35) as an average over an ensemble of systems. If single-parameter finite-size scaling holds for $Q_{\text {typ }}$ and $Q_{\text {typ }}$ is scale invariant at the critical point then close to the critical point

$$
Q_{\mathrm{typ}}(l)=Q_{\mathrm{typ}}^{*}+a l^{1 / \nu} t
$$

The box-observable $\tilde{Q}_{\text {typ }}=Q_{\text {typ }}-Q_{\text {typ }}^{*}$ thus scales like

$$
\tilde{Q}_{\text {typ }} \propto l^{1 / \nu}
$$

close to the critical point. Identifying Eqs. (9.35) and (9.37) relates the critical exponent $\nu$ of the localization length to the multifractal exponents $\alpha_{0}^{[Q]}$ and $X^{[Q]}$,

$$
1 / \nu=\alpha_{0}^{[Q]}+X^{[Q]}
$$

Fastenrath, Janßen, and Pook (1992) calculated Thouless numbers near the center of the lowest Landau level for systems of varying size. They found that the typical Thouless number $g_{\text {typ }}(l)=\exp [\langle\ln g(l)\rangle]$ scales according to Eq. (9.35) with $\alpha_{0}^{[g]}=2.25 \pm 0.05$ and $X^{[g]}=-1.75 \pm 0.05$. Using Eq. (9.38), $\nu=2.2 \pm 0.3$ in accordance with Eq. (8.4).

\section{DISCUSSION AND CONCLUSIONS}

Having reviewed the experimental and theoretical results we are now in a position to compare both. The most important result of the comparison is that both experiments and numerical simulations show similar scaling behavior. The plateau transition in the integer 
QHE can thus be understood as a continuous phase transition of noninteracting electrons with a single diverging length $\xi$. If we focus on spin-split Landau levels there is a remarkable agreement in the value of the exponent $\nu$, describing the divergence of the localization length $\xi$, between experiment [2.3 \pm 0.1 (Koch et al., 1991b), $2.4 \pm 0.1$ (Wang et al., 1994a)] and numerical simulation $[2.35 \pm 0.03$ (Huckestein, 1992)]. This suggests that the temperatures in these experiments are in fact low enough to observe the critical behavior and influences of the finite width of the Fermi-Dirac distribution are negligible.

From the value of $\nu$ and the value of $1 / \nu z=0.41 \pm 0.04$ measured in the dynamic scaling experiment of Engel et al. (1993) we get the dynamic critical exponent $z=1.0 \pm 0.1$. Dynamical scaling can also be used to explain the temperature dependence of the transition. In the approach outlined in Sec. IIA the temperature sets the effective system size $L_{\text {eff }} \propto T^{-p / 2}$ that has to be compared to the localization length $\xi \propto|\Delta E|^{-\nu}$. In this interpretation the measured temperature exponent $\kappa=p / 2 \nu$. In the dynamic scaling interpretation the temperature enters as the frequency range $\omega=k_{B} T / \hbar$ over which excitations are present in the system. Scaling as a function of $\omega \tau$ with $\tau \propto \xi^{z}$ leads to the identification $\kappa=1 / \nu z$, in agreement with the results of Wei et al. $(1988 ; 1990)$ from experiments on InGaAs/InP heterostructures and on AlGaAs/GaAs heterostructures (1992). Koch et al. (1991a; 1991b), using AlGaAs/GaAs heterostructures, however, find values of $\kappa$, which vary between samples. Wei et al. (1992) argue that there is a characteristic temperature below which universal scaling behavior is observed and that this temperature is much lower in AlGaAs/GaAs than in InGaAs/InP. The agreement between the dynamic scaling experiment and the temperature scaling below the characteristic temperature suggests that these experiments are indeed probing the asymptotic low-temperature regime described by the dynamic exponent $z$. Which interpretation describes the experiments correctly presumably depends on the relative size of $L_{\text {eff }} / \xi$ and $k_{B} T \tau / \hbar$.

We then need to understand why other experiments see power-law scaling with temperature but with nonuniversal exponents. This difference could be due to the different ranges of the disorder potentials in the different materials. In InGaAs/InP the electron gas 
is formed in the InGaAs, which is an alloy with a potential presumably varying on an atomic length scale. By contrast, in AlGaAs/GaAs heterostructures the electron gas is situated in the GaAs, which forms a perfect crystal. In conventional heterostructures, doped in the AlGaAs to increase the mobility, the major scattering mechanism is remote ionized donor scattering. The samples used in the experiments by Koch et al. were additionally doped in the plane of the electron gas. Since this introduces charged impurities into the quasitwo-dimensional gas it is not obvious that the range of the scatterers is actually sufficiently different in both materials. An increase in the correlation length of the disorder potential leads to an increase in the irrelevant length scale $\xi_{\text {irr }}$ and hence to an increase in finite-size effects.

The temperature dependence of different phase-breaking mechanism could also account for different characteristic temperatures. At sufficiently low temperatures phase-breaking occurs presumably due to a single process, Coulomb interaction between the electrons, associated with the dynamical exponent $z$. At higher temperatures other processes, like the electron-phonon interaction, become important. Since these processes will, in general, have different temperature dependences the observed nonuniversality of $\kappa$ might be a crossover phenomenon. The characteristic temperature of the crossover to the asymptotic, universal low-temperature regime is nonuniversal and might show a strong sample dependence.

In addition to the relevant length scale $\xi$ the numerical simulations show the influence of an irrelevant length scale $\xi_{\text {irr }}$. For higher Landau levels and short correlation length of the disorder the irrelevant length can become orders of magnitude larger than the magnetic length and hence comparable to the phase coherence length in experiments. On length scales smaller than $\xi_{\text {irr }}$ the scaling is described by a two-parameter flow according to Eq. (8.13) while the asymptotic scaling behavior for large length scales is given by a single-parameter scaling relation. In a $\sigma_{x x}$ vs. $\sigma_{x y}$ flow diagram we expect the renormalization group flow to produce a set of different curves in the former regime and a single scaling curve in the latter. The differences in the flow diagrams of Wei et al. (1987), of Yamane et al. (1989), of Kravchenko et al. (1990), and of McEuen et al. (1990) might have their origin in the 
different size of the crossover length scale.

The value $z=1$ extracted from the experiments does not agree with the result $z=2$ for noninteracting electrons. This means that another mechanism is necessary to describe the dynamics of the system. It has been argued by Polyakov and Shklovskii (1993) and by Lee, Wang, and Kivelson (1993) that the Coulomb interaction between the electrons gives rise to $z=1$. This can be motivated by the naive scaling argument that the relevant energy scale is the Coulomb energy on the length scale $\xi, E \propto e^{2} / \xi$, which immediately provides $z=1$. If Coulomb interactions have to be taken into account to understand the dynamic scaling behavior, this raises the question whether these interactions are relevant for the static scaling exponent $\nu$, too. The agreement between experiments and simulations for noninteracting electrons suggests that $\nu$ is not changed by Coulomb interactions. However, no calculation of $\nu$ for interacting electrons under quantum Hall conditions has been performed so far, although Lee, Wang, and Kivelson (1993) claim that the edge states of interacting quantum Hall droplets behave like noninteracting particles and that the results of the network model apply in presence of interactions as well.

For spin-degenerate Landau levels the measured values of $\nu$ do not agree with the numerical value $\nu=2.35$ (Koch et al., 1991b). Also the measured value of $\kappa$ is much smaller in the spin-degenerate case (Wei et al., 1988; Koch et al., 1991a; Engel et al., 1993). The data analysis in all experimental investigations assumed a single critical energy in the center of the spin-degenerate Landau level. Polyakov and Shklovskii (1993), Lee and Chalker (1994), and Wang, Lee, and Wen (1994b) argued recently for the existence of two separate critical energies even in the completely spin-degenerate case. Lee and Chalker (1994), and Wang, Lee, and Wen (1994b) supported their arguments with numerical results that were compatible with two critical energies and $\nu=2.35$. When fitting their data with a single critical energy, they observed a divergence of $\xi$ with an exponent $\approx 5.8$ in some intermediate range of energies. It has to be seen whether experimental data can also be fitted with two critical energies and $\nu=2.35$.

As far as the value of the conductivity tensor at the transition is concerned there is 
not much agreement between experiment and theory. While theoretical arguments and calculations seem to favor $\sigma_{x x}=0.5 e^{2} / h$ and $\sigma_{x y}=(n+1 / 2) e^{2} / h$ (at least in the lowest Landau level), the experimental situation is completely unclear.

So far, there exists no direct experimental evidence for the multifractal structure of eigenfunctions at the transition. Since the multifractality leads to an anomalous behavior of the density-response function signatures of multifractality might be observable in the temperature dependence of transport processes (Chalker and Daniell, 1988; Brandes et al., 1994).

In summary, we have shown that numerical simulations are a suitable instrument to calculate the critical behavior of noninteracting electrons in the quantum Hall system. The observed behavior agrees with the description of the plateau transitions as continuous phase transitions with a single diverging length scale. The numerically determined critical exponent $\nu=2.35 \pm 0.03$ agrees well with experimental results. In addition to the relevant scaling field driving the transition an irrelevant scaling field is observed in the numerical simulations. The associated crossover length can become macroscopic, in which case the irrelevant scaling field has to be included in the scaling flow.

\section{ACKNOWLEDGMENTS}

I would like to thank everybody who had part in the completion of this work: The colleagues and friends from whom I learned so much. My collaborators who helped me perform the calculations presented here. Hans A. Weidenmüller for giving me the opportunity and encouraging me to write this review. Gregor Hackenbroich, Felix von Oppen, Ludwig Schweitzer, and Petra Wild for their help in the final stages of this work. And last, but not least, my family. Without their patience, support, and love I could never have done it. 


\section{APPENDIX: RANDOM-LANDAU-MATRIX-MODEL}

The generalization of the result for the correlation function Eq. (3.24) to arbitrary Landau level index $n$ was given by Mieck (1993),

$$
\begin{aligned}
& \overline{\left\langle n k_{1}|V| n k_{2}\right\rangle\left\langle n k_{3}|V| n k_{4}\right\rangle}=\frac{V_{0}^{2}}{\sqrt{2 \pi} l_{c} L_{y} \beta} \exp \left(-\frac{1}{2}\left(k_{1}-k_{2}\right)^{2} l_{c}^{2} \beta^{2}\right) \exp \left(-\frac{1}{2}\left(k_{1}-k_{4}\right)^{2} l_{c}^{2} \frac{1}{\beta^{2}}\right) \\
& \delta_{k_{1}-k_{2}, k_{4}-k_{3}} \times \sum_{k=0}^{n} \sum_{m=0}^{n} \sum_{l=0}^{\min (2 k, 2 m)} 2^{-l}\left(\begin{array}{c}
2 k \\
l
\end{array}\right)\left(\begin{array}{c}
2 m \\
l
\end{array}\right) \frac{l !(k+m-l) !}{k ! m !} \beta^{-2(m+k-l)} \\
& \times L_{n-k}^{-1 / 2}\left(\frac{\left(k_{1}-k_{2}\right)^{2} l_{c}^{2}}{2}\right) L_{n-m}^{-1 / 2}\left(\frac{\left(k_{1}-k_{2}\right)^{2} l_{c}^{2}}{2}\right) L_{k+m-l}^{-1 / 2}\left(-\frac{1}{2} \frac{\left(k_{1}-k_{4}\right)^{2} l_{c}^{2}}{\beta^{2}\left(\beta^{2}-1\right)}\right) .
\end{aligned}
$$

It is instructive to study the dependence of the correlation function on the correlation length of the disorder potential and the Landau level index. As the correlation length and hence $\beta$ increases, the off-diagonal matrix elements become smaller but the range of the correlations along the diagonals increases. In higher Landau levels additional polynomials enter the correlation function (A1) due to the presence of the Hermite polynomials in the harmonic oscillator functions. While in the lowest Landau level the correlations (3.24) are always positive, in higher Landau levels the correlations start to oscillate for short correlation lengths and small differences $k_{1}-k_{4}$. In the limit of large $\sigma / l_{c}$, the correlations become independent of the Landau level and take on the universal form given by the expression for the lowest Landau level, Eq. (3.24). This limit corresponds to the semiclassical limit discussed in Section IIID that is independent of the Landau level index.

In order to use the Ansatz (3.28) for the matrix elements $\left\langle n_{1} k_{1}|V| n_{2} k_{2}\right\rangle$ the weight function $h(x, k)$ appearing in it has to be expressed through the correlation function $\bar{g}(x, k)$ of the disorder potential. Using the translational invariance of the disorder-potential correlation function and the fact that the disorder potential is real, the weight function $h(x, k)$ is seen to be a real symmetric function,

$$
h(x, k)=h(-x, k)=h(x,-k)=h(-x,-k) .
$$

With Eq. (3.21), $h(x, k)$ is related to the correlation function $\bar{g}(x, k)$ by 


$$
\bar{g}\left(x_{1}-x_{2}, k\right)=\int d x^{\prime} h\left(x_{1}-x^{\prime}, k\right) h\left(x_{2}-x^{\prime}, k\right)
$$

which can easily be solved by Fourier transformation to give

$$
\tilde{g}(q, k)=[\tilde{h}(q, k)]^{2}
$$

where $\tilde{g}(q, k)$ and $\tilde{h}(q, k)$ are the Fourier transforms with respect to $x$ of $\bar{g}(x, k)$ and $h(x, k)$, respectively. For the simple case of Gaussian correlations we find that $h(x, k)$ is given by

$$
h(x, k)=\frac{V_{0}}{\sqrt{2 \pi L_{y}} \sigma} e^{-k^{2} \sigma^{2} / 4} e^{-x^{2} / \sigma^{2}},
$$

and Eq. (3.28) becomes

$$
\begin{aligned}
& \left\langle n_{1} k_{1}|V| n_{2} k_{2}\right\rangle=\frac{V_{0} \beta l_{c}}{\sqrt{2 \pi L_{y}} \sigma} \exp \left(\frac{\left(k_{1}-k_{2}\right)^{2} l_{c}^{2} \beta^{2}}{4}\right)\left(2^{\left(n_{1}+n_{2}\right)} n_{1} ! n_{2} ! \pi\right)^{-1 / 2} \\
& \times \int d \xi u_{0}\left(\beta \xi+\frac{\left(k_{1}+k_{2}\right) l_{c}}{2},\left(k_{1}-k_{2}\right) l_{c}\right) \exp \left(-\xi^{2}\right) \\
& \times \int d \eta \exp \left(-\frac{l_{c}^{2}+\sigma^{2}}{\sigma^{2}} \eta\right) H_{n_{1}}\left(\eta+\frac{\xi}{\beta}-\frac{\left(k_{1}-k_{2}\right) l_{c}}{2}\right) H_{n_{2}}\left(\eta+\frac{\xi}{\beta}+\frac{\left(k_{1}-k_{2}\right) l_{c}}{2}\right),
\end{aligned}
$$

which gives Eq. (3.30) for $n_{1}=n_{2}=0$.

For numerical calculations, the integration in Eq. (3.30) needs to be discretized. Huckestein and Kramer (1989) chose a step width of $\Delta X / 2 l_{c}$, where $\Delta X=\Delta k l_{c}^{2}=2 \pi l_{c}^{2} / L_{y}$ is the difference in the center coordinate of neighboring Landau wavefunctions. The discrete version of Eq. (3.30) then reads

$$
\begin{aligned}
\left\langle 0 k_{1}|V| 0 k_{2}\right\rangle= & \frac{V_{0}}{\left(\sqrt{2 \pi} l_{c} L_{y} \Sigma\right)^{1 / 2}} \exp \left(\frac{\left(k_{1}-k_{2}\right)^{2} l_{c}^{2} \beta^{2}}{4}\right) \\
& \times \sum_{i} u_{2 k_{1}+i, k_{2}-k_{1}} \exp \left(-\frac{\pi^{2} l_{c}^{2}}{L_{y}^{2} \beta^{2}} i^{2}\right)
\end{aligned}
$$

with

$$
\Sigma=\sum_{i} \exp \left(-2 \frac{\pi^{2} l_{c}^{2}}{L_{y}^{2} \beta^{2}} i^{2}\right)
$$

The limits on the summation over $i$ can be chosen such that the influence of the neglected terms is less than the statistical fluctuation due to disorder. 
It remains to specify the distribution function of the random variables $u_{x, k}$. To correspond to a Gaussian distribution in real space they should also be taken from a Gaussian distribution. Numerically, it is faster to generate random numbers with a uniform distribution. While the distribution function of the matrix elements seems to strongly influence finite-size effects for the three-dimensional Anderson model (Kramer et al., 1990; MacKinnon, 1994), in the present case no noticeable differences could be observed between Gaussian and uniform distributions of the same variance.

A similar approach to that presented above was developed by Weidenmüller and Mieck (Weidenmüller, 1987; Mieck, 1990; Mieck and Weidenmüller, 1991; Mieck, 1993). Central to their idea is the observation that operators projected onto a single Landau level can be expanded in matrix polynomials that are related to the magnetic translation operators (Weidenmüller, 1987). For the correlation function of the disorder potential this expansion proceeds as follows. Eq. (3.19) can be rewritten in terms of the Fourier transform $\tilde{g}(\mathbf{p})$ of $g\left(\mathbf{r}-\mathbf{r}^{\prime}\right)$, 闯

$$
\begin{aligned}
& \overline{\left\langle n_{1} k_{1}|V| n_{2} k_{2}\right\rangle\left\langle n_{3} k_{3}|V| n_{4} k_{4}\right\rangle}=\int \frac{d^{2} p}{2 \pi} \tilde{g}(|\mathbf{p}|) \\
& \times\left\langle n_{1} k_{1}\left|\exp \left[i \mathbf{p} \cdot \mathbf{r}_{1}\right]\right| n_{2} k_{2}\right\rangle\left\langle n_{3} k_{3}\left|\exp \left[-i \mathbf{p} \cdot \mathbf{r}_{2}\right]\right| n_{4} k_{4}\right\rangle .
\end{aligned}
$$

The matrix elements of the plane waves can be expressed using the magnetic translation operators. These are generated by the operator $\mathcal{P}$ that complements the canonical momentum $\boldsymbol{\Pi}=\mathbf{p}-\mathbf{e A}$. In the gauge $\mathbf{A}=B\left(-(1-\alpha) y \hat{\mathrm{e}}_{x}+\alpha x \hat{\mathrm{e}}_{y}\right)$ these two operators are given by

$$
\boldsymbol{\Pi}=\left(\begin{array}{c}
p_{x}+e B(1-\alpha) y \\
p_{y}-e B \alpha x
\end{array}\right), \quad \mathcal{P}=\left(\begin{array}{c}
p_{x}-e B \alpha y \\
p_{y}+e B(1-\alpha) x
\end{array}\right) .
$$

The commutation relations for these operators are those of the two-dimensional harmonic oscillator where the canonical momentum $\boldsymbol{\Pi}$ is diagonal in the degenerate, internal states $k$ of each Landau level while $\mathcal{P}$ is diagonal between the Landau bands $n$,

$$
\left[\Pi_{x}, \Pi_{y}\right]=i e B \hbar,\left[\mathcal{P}_{x}, \mathcal{P}_{y}\right]=-i e B \hbar,[\Pi, \mathcal{P}]=0
$$

\footnotetext{
${ }^{4}$ For simplicity, we discuss the limit $L_{y} \rightarrow \infty$ in the following.
} 
With these operators $i \mathbf{p} \cdot \mathbf{r}$ can be expressed as

$$
i \mathbf{p} \cdot \mathbf{r}=\frac{i}{e B}\left(\hat{\mathrm{e}}_{z} \times \mathbf{p}\right)(\mathcal{P}-\mathbf{\Pi})
$$

Using the commutation relations Eq. (A11) this can be exponentiated to give

$$
\begin{aligned}
\exp [i \mathbf{p} \cdot \mathbf{r}] & =\exp \left[(i / e B)\left(\hat{\mathrm{e}}_{z} \times \mathbf{p}\right)(\mathcal{P}-\boldsymbol{\Pi})\right] \\
& =\exp \left[(i / e B)\left(\hat{\mathrm{e}}_{z} \times \mathbf{p}\right) \mathcal{P}\right] \exp \left[-(i / e B)\left(\hat{\mathrm{e}}_{z} \times \mathbf{p}\right) \boldsymbol{\Pi}\right]
\end{aligned}
$$

The matrix elements of $\exp [i \mathbf{p} \cdot \mathbf{r}]$ are then given by the matrix elements $U_{k_{1}, k_{2}}(\mathbf{p})=$ $\left\langle n_{1} k_{1}\left|\exp \left[i / e B\left(\hat{\mathrm{e}}_{z} \times \mathbf{p}\right) \mathcal{P}\right]\right| n_{2} k_{2}\right\rangle$ and $W_{n_{1}, n_{2}}(\mathbf{p})=\left\langle n_{1} k_{1}\left|\exp \left[-i / e B\left(\hat{\mathrm{e}}_{z} \times \mathbf{p}\right) \mathbf{\Pi}\right]\right| n_{2} k_{2}\right\rangle$,

$$
\left\langle n_{1} k_{1}|\exp [i \mathbf{p} \cdot \mathbf{r}]| n_{2} k_{2}\right\rangle=U_{k_{1}, k_{2}}(\mathbf{p}) W_{n_{1}, n_{2}}(\mathbf{p})
$$

Note that due to the commutation relations, $U_{k_{1}, k_{2}}(\mathbf{p})$ does not depend on the Landau-level index, and $W_{n_{1}, n_{2}}(\mathbf{p})$ does not depend on the internal quantum numbers $k$. Using Eq. (A13), the correlation function (A9) can now be expressed in terms of the matrix polynomials

$$
\begin{aligned}
\overline{\left\langle n_{1} k_{1}|V| n_{2} k_{2}\right\rangle\left\langle n_{3} k_{3}|V| n_{4} k_{4}\right\rangle}= & \int \frac{d^{2} p}{2 \pi} \tilde{g}(|\mathbf{p}|) \\
& \times U_{k_{1}, k_{2}}(\mathbf{p}) W_{n_{1}, n_{2}}(\mathbf{p}) U_{k_{3}, k_{4}}(-\mathbf{p}) W_{n_{3}, n_{4}}(-\mathbf{p}) .
\end{aligned}
$$

The particular form of the matrix polynomials depends on the choice of the gauge. For the Landau gauge (3.13) they are given by (Mieck, 1993)

$$
\begin{gathered}
U_{k, k^{\prime}}(\mathbf{p})=\exp \left[i p_{x}\left(k+k^{\prime}\right) / 2\right] \delta\left(p_{x}-k+k^{\prime}\right) \\
W_{n, n^{\prime}}(\mathbf{p})=i^{|\Delta n|}\left(\frac{\nu !}{(\nu+|\Delta n|) !}\right)^{1 / 2} e^{-|\mathbf{p}|^{2} / 4}(|\mathbf{p}| / \sqrt{2})^{|\Delta n|} L_{\nu}^{|\Delta n|}\left(|\mathbf{p}|^{2} / 2\right) e^{-i\left(n^{\prime}-n\right) \phi}, \\
\nu=\min \left(n, n^{\prime}\right), \tan \phi=p_{y} / p_{x} .
\end{gathered}
$$

The delta function in the matrix polynomial $U_{k, k^{\prime}}(\mathbf{p})$ reproduces the delta function that we already observed in the correlation function (3.21).

The matrix-polynomial representation (A14) of the plane-wave operator can also be used to generate the matrix elements $\left\langle n_{1} k_{1}|V| n_{2} k_{2}\right\rangle$. For this, we express the real space potential $V(\mathbf{r})$ by its Fourier transform $\tilde{V}(\mathbf{k})$, 


$$
V(\mathbf{r})=\int \frac{d^{2} k}{2 \pi} e^{-i \mathbf{k} \cdot \mathbf{r}} \tilde{V}(\mathbf{k})
$$

Using Eq. (3.18) the second moment of the Fourier transform is given by

$$
\overline{\tilde{V}\left(\mathbf{k}_{\mathbf{1}}\right) \tilde{V}\left(\mathbf{k}_{\mathbf{2}}\right)}=2 \pi \tilde{g}\left(\left|\mathbf{k}_{\mathbf{1}}\right|\right) \delta\left(\mathbf{k}_{1}+\mathbf{k}_{2}\right) .
$$

Thus the Fourier transform of the random potential is $\delta$-correlated and can be expressed by Gaussian random variables $\tilde{v}(\mathbf{k})$ of unit variance,

$$
\tilde{V}(\mathbf{k})=(2 \pi)^{1 / 2}[\tilde{g}(|\mathbf{k}|)]^{1 / 2} \tilde{v}(\mathbf{k}) .
$$

The potential $V(\mathbf{r})$ is then determined by $\tilde{v}(\mathbf{k})$,

$$
V(\mathbf{r})=\int \frac{d^{2} k}{(2 \pi)^{1 / 2}}[\tilde{g}(|\mathbf{k}|)]^{1 / 2} e^{-i \mathbf{k} \cdot \mathbf{r}} \tilde{v}(\mathbf{k})
$$

where the second moment of $\tilde{v}(\mathbf{k})$ is given by

$$
\overline{\tilde{v}^{*}\left(\mathbf{k}_{1}\right) \tilde{v}\left(\mathbf{k}_{2}\right)}=\delta\left(\mathbf{k}_{1}-\mathbf{k}_{2}\right)
$$

Using Eq. (A14) the matrix elements of the disorder potential can now be expressed in terms of the random variables $\tilde{v}(\mathbf{k})$ and the matrix polynomials,

$$
\left\langle n_{1} k_{1}|V| n_{2} k_{2}\right\rangle=\int \frac{d^{2} p}{(2 \pi)^{1 / 2}}[\tilde{g}(|\mathbf{p}|)]^{1 / 2} U_{n_{1} n_{2}}(-\mathbf{p}) W_{k_{1} k_{2}}(-\mathbf{p}) \tilde{v}(\mathbf{p}) .
$$

\footnotetext{
${ }^{5}$ Note that since $V(\mathbf{r})$ is real $\tilde{v}^{*}(\mathbf{k})=\tilde{v}(-\mathbf{k})$.
} 


\section{REFERENCES}

[1] Abrahams, E., P. W. Anderson, D. C. Licciardello, and V. Ramakrishnan, 1979, Phys. Rev. Lett. 42, 673.

[2] Altshuler, B. L., 1985, Pis'ma Zh. Eksp. Teor. Fiz. 41, 530, [JETP Lett. 41, 648 (1985)].

[3] Altshuler, B. L., V. E. Kravtsov, and I. V. Lerner, 1986, Pis'ma Zh. Eksp. Teor. Fiz. 43, 342, [JETP Lett. 43, 441 (1986)].

[4] Altshuler, B. L., V. E. Kravtsov, and I. V. Lerner, 1989, Phys. Lett. A 134, 488.

[5] Anderson, P. W., 1958, Phys. Rev. 109, 1492.

[6] Anderson, P. W., D. J. Thouless, E. Abrahams, and D. S. Fisher, 1980, Phys. Rev. B 22, 3519 .

[7] Ando, T., 1983, J. Phys. Soc. Jpn. 52, 1740.

[8] Ando, T., 1984a, J. Phys. Soc. Jpn. 53, 3101.

[9] Ando, T., 1984b, J. Phys. Soc. Jpn. 53, 3126.

[10] Ando, T., 1989a, Phys. Rev. B 40, 5325.

[11] Ando, T., 1989b, Phys. Rev. B 40, 9965.

[12] Ando, T., 1992, J. Phys. Soc. Jpn. 61, 415.

[13] Ando, T. and H. Aoki, 1985, J. Phys. Soc. Jpn. 54, 2238.

[14] Ando, T., Y. Matsumoto, and Y. Uemura, 1975, J. Phys. Soc. Jpn. 39, 279.

[15] Ando, T. and Y. Uemura, 1974, J. Phys. Soc. Jpn. 36, 959.

[16] Aoki, H., 1977, J. Phys. C 10, 2583.

[17] Aoki, H., 1978, J. Phys. C 11, 3823. 
[18] Aoki, H., 1983, J. Phys. C 16, L205.

[19] Aoki, H., 1985, J. Phys. C 18, L67.

[20] Aoki, H., 1986, Phys. Rev. B 33, 7310.

[21] Aoki, H. and T. Ando, 1981, Solid State Commun. 38, 1079.

[22] Aoki, H. and T. Ando, 1985, Phys. Rev. Lett. 54, 831.

[23] Aoki, H. and T. Ando, 1986, Phys. Rev. Lett. 57, 3093.

[24] Arovas, D. P., R. N. Bhatt, F. D. M. Haldane, P. B. Littlewood, and R. Rammal, 1988, Phys. Rev. Lett. 60, 619.

[25] Avron, J. E., R. Seiler, and B. Simon, 1983, Phys. Rev. Lett. 51, 51.

[26] Azbel', M. Y., 1964, Zh. Eksp. Teo. Fiz. 46, 929, [Sov. Phys. JETP 19, 634 (1964)].

[27] Barber, M. N., 1983, in Phase Transistions and Critical Phenomena, Vol. 8, edited by C. Domb and J. Lebowitz, (Academic Press, London), p. 146.

[28] Bauer, J., T.-M. Chang, and J. L. Skinner, 1990, Phys. Rev. B 42, 8121.

[29] Black, J. L. and V. J. Emery, 1981, Phys. Rev. B 23, 429.

[30] Brandes, T., L. Schweitzer, and B. Kramer, 1994, Phys. Rev. Lett. 72, 3582.

[31] Brézin, E., 1982, J. Phys. (Paris) 43, 15.

[32] Brézin, E., D. J. Gross, and C. Itzykson, 1984, Nucl. Phys. B 235[FS11], 24.

[33] Brézin, E., S. Hikami, and J. Zinn-Justin, 1980, Nucl. Phys. B 165, 528.

[34] Cardy, J. L., 1984, J. Phys. A 17, L385.

[35] Chalker, J. T., 1987, J. Phys. C 20, L493.

[36] Chalker, J. T. and P. D. Coddington, 1988, J. Phys. C 21, 2665. 
[37] Chalker, J. T. and G. J. Daniell, 1988, Phys. Rev. Lett. 61, 593.

[38] Chalker, J. T. and J. P. G. Eastmond, 1993, (unpublished).

[39] Chhabra, A. and R. V. Jensen, 1989, Phys. Rev. Lett. 62, 1327.

[40] den Nijs, M. P. M., 1979, J. Phys. A 12, 1857.

[41] Duplantier, B. and A. W. W. Ludwig, 1991, Phys. Rev. Lett. 66, 247.

[42] Edwards, J. T. and D. J. Thouless, 1972, J. Phys. C 5, 807.

[43] Efetov, K. B., 1983, Adv. Phys. 32, 53.

[44] Efetov, K. B., A. I. Larkin, and D. E. Khmel'nitskii, 1980, Zh. Eksp. Teo. Fiz. 79, 1120, [Sov. Phys. JETP 52, 568 (1980)].

[45] Engel, L. W., D. Shahar, Ç. Kurdak, and D. C. Tsui, 1993, Phys. Rev. Lett. 71, 2638.

[46] Engel, L. W., H. P. Wei, D. C. Tsui, and M. Shayegan, 1990, Surf. Sci. 229, 13.

[47] Fastenrath, U., M. Janßen, and W. Pook, 1992, Physica A 191, 401.

[48] Fertig, H. A., 1988, Phys. Rev. B 38, 996.

[49] Fisher, M. P. A., 1990, Phys. Rev. Lett. 65, 923.

[50] Fisher, M. P. A., G. Grinstein, and S. M. Girvin, 1990, Phys. Rev. Lett. 64, 587.

[51] Haldane, F. D. M. and E. H. Rezayi, 1985, Phys. Rev. B 31, 2529.

[52] Halperin, B. I., 1982, Phys. Rev. B 25, 2185.

[53] Halsey, T. C., M. H. Jensen, L. P. Kadanoff, I. Procaccia, and B. Shraiman, 1986, Phys. Rev. A 33, 1141.

[54] Hentschel, H. G. E. and I. Procaccia, 1983, Physica 8D, 435.

[55] Hikami, S., 1986, Prog. Theor. Phys. 76, 1210. 
[56] Hofstadter, D. R., 1976, Phys. Rev. B 14, 2239.

[57] Huckestein, B., 1990, Physica A 167, 175.

[58] Huckestein, B., 1992, Europhysics Lett. 20, 451.

[59] Huckestein, B., 1993, (unpublished).

[60] Huckestein, B., 1994, Phys. Rev. Lett. 72, 1080.

[61] Huckestein, B. and B. Kramer, 1989, Solid State Comm. 71, 445.

[62] Huckestein, B. and B. Kramer, 1990, Phys. Rev. Lett. 64, 1437.

[63] Huckestein, B. and B. Kramer, 1992, in High Magnetic Fields in Semiconductor Physics III, edited by G. Landwehr, Springer Series in Solid State Sciences No. 101, (SpringerVerlag, Berlin), p. 70.

[64] Huckestein, B., B. Kramer, R. Meisels, K. Y. Lim, F. Kuchar, G. Weimann, and W. Schlapp, 1991, in Proceedings of the 20th International Conference 'The Physics of Semiconductors', Thessaloniki, Greece, August 6-10, 1990, edited by E. M. Anastassakis and J. D. Joannopoulos, World Scientific, p. 849.

[65] Huckestein, B., B. Kramer, and L. Schweitzer, 1992, Surf. Sci. 263, 125.

[66] Huckestein, B. and L. Schweitzer, 1992a, Helvetica Physica Acta 65, 317.

[67] Huckestein, B. and L. Schweitzer, 1992b, Physica A 191, 406.

[68] Huckestein, B. and L. Schweitzer, 1994, Phys. Rev. Lett. 72, 713.

[69] Huo, Y. and R. N. Bhatt, 1992, Phys. Rev. Lett. 68, 1375.

[70] Huo, Y., R. E. Hetzel, and R. N. Bhatt, 1993, Phys. Rev. Lett. 70, 481.

[71] Hwang, S. W., H. P. Wei, L. W. Engel, D. C. Tsui, and A. M. M. Pruisken, 1993, Phys. Rev. B 48, 11416. 
[72] Iordansky, S. V., 1982, Solid State Commun. 43, 1.

[73] Jaeger, L., 1991, J. Phys.: Condens. Matter 3, 2441.

[74] Janßen, M., 1994, Int. J. Mod. Phys. B 8, 943.

[75] Kawaji, S. and J. Wakabayashi, 1981, in Proc. Oji Int. Seminar, Hakone, 1980, Physics in High Magnetic Fields, edited by S. Chikazumi and N. Miura, (Springer, Berlin), p. 284.

[76] Kawaji, S. and J. Wakabayashi, 1987, J. Phys. Soc. Jpn. 56, 21.

[77] Kazarinov, R. F. and S. Luryi, 1982, Phys. Rev. B 25, 7626.

[78] Ketzmerick, R., G. Petschel, and T. Geisel, 1992, Phys. Rev. Lett. 69, 695.

[79] Khmel'nitskii, D. E., 1983, Pis'ma Zh. Eksp. Teor. Fiz. 38, 454, [JETP Lett. 38, 552 (1984)].

[80] Koch, S., R. J. Haug, K. von Klitzing, and K. Ploog, 1991a, Phys. Rev. B 43, 6828.

[81] Koch, S., R. J. Haug, K. von Klitzing, and K. Ploog, 1991b, Phys. Rev. Lett. 67, 883.

[82] Kohmoto, M., 1985, Ann. Phys. (NY) 160, 343.

[83] Kramer, B., K. Broderix, A. MacKinnon, and M. Schreiber, 1990, Physica A 167, 163.

[84] Kramer, B. and A. MacKinnon, 1993, Rep. Prog. Phys. 56, 1469.

[85] Kravchenko, S. V., B. K. Medvedev, V. G. Mokerov, V. M. Pudalov, D. A. Rinberg, and S. G. Semenchinsky, 1990, Surf. Sci. 229, 63.

[86] Kravchenko, S. V. and V. M. Pudalov, 1989, Pis'ma Zh. Eksp. Teor. Fiz. 50, 65, [JETP Lett. 50, 73 (1989)].

[87] Kuchar, F., R. Meisels, G. Weimann, and W. Schlapp, 1986, Phys. Rev. B 38, 2965.

[88] Langer, J. S. and T. Neal, 1966, Phys. Rev. Lett. 16, 984. 
[89] Laughlin, R. B., 1981, Phys. Rev. B 23, 5632.

[90] Lee, D.-H., S. Kivelson, and S.-C. Zhang, 1992, Phys. Rev. Lett. 68, 2386.

[91] Lee, D.-H., Z. Wang, and S. Kivelson, 1993, Phys. Rev. Lett. 70, 4130.

[92] Lee, D. K. K. and J. T. Chalker, 1994, Phys. Rev. Lett. 72, 1510.

[93] Lee, P. A. and T. V. Ramakrishnan, 1985, Rev. Mod. Phys. 57, 287.

[94] Lee, P. A. and A. D. Stone, 1985, Phys. Rev. Lett. 54, 1622.

[95] Levine, H., S. B. Libby, and A. M. M. Pruisken, 1983, Phys. Rev. Lett. 51, 1915.

[96] Levine, H., S. B. Libby, and A. M. M. Pruisken, 1984a, Nucl. Phys. B 240[FS12], 30.

[97] Levine, H., S. B. Libby, and A. M. M. Pruisken, 1984b, Nucl. Phys. B 240[FS12], 49.

[98] Levine, H., S. B. Libby, and A. M. M. Pruisken, 1984c, Nucl. Phys. B 240[FS12], 71.

[99] Licciardello, D. C. and D. J. Thouless, 1975, J. Phys. C 8, 4157.

[100] Liu, D. and S. Das Sarma, 1993, Mod. Phys. Lett. B 7, 449.

[101] Liu, D. and S. Das Sarma, 1994, Phys. Rev. B 49, 2677.

[102] Ludwig, A. W. W., 1990, Nucl. Phys. B 330, 639.

[103] Luttinger, J. M., 1951, Phys. Rev. 84, 814.

[104] MacKinnon, A., 1994, J. Phys.: Condens. Matter 6, 2511.

[105] MacKinnon, A. and B. Kramer, 1981, Phys. Rev. Lett. 47, 1546.

[106] MacKinnon, A. and B. Kramer, 1983, Z. Phys. B 53, 1.

[107] Mandelbrot, B. B., 1983, The Fractal Geometry of Nature, (W. H. Freeman, San Francisco).

[108] McEuen, P. L., A. Szafer, C. A. Richter, B. W. Alphenaar, J. K. Jain, A. D. Stone, 
R. G. Wheeler, and R. N. Sacks, 1990, Phys. Rev. Lett. 64, 2062.

[109] Mieck, B., 1990, Europhysics Lett. 13, 453.

[110] Mieck, B., 1993, Z. Phys. B 90, 427.

[111] Mieck, B. and H. A. Weidenmüller, 1991, Z. Phys. B 84, 59.

[112] Mil'nikov, G. V. and I. M. Sokolov, 1988, Pis'ma Zh. Eksp. Teor. Fiz. 48, 494, [JETP Lett. 48, 536 (1988)].

[113] Niu, Q., D. J. Thouless, and Y. S. Wu, 1985, Phys. Rev. B 31, 3372.

[114] Ono, Y., 1982a, in Anderson Localization, edited by Y. Nagaoka and H. Fukuyama, Springer Series in Solid State Sciences No. 39, (Springer, Berlin), p. 207.

[115] Ono, Y., 1982b, J. Phys. Soc. Jpn. 51, 2055.

[116] Oseledec, V. I., 1968, Trans. Moscow Math. Soc. 19, 197.

[117] Paalanen, M. A., D. C. Tsui, and A. C. Gossard, 1982, Phys. Rev. B 25, 5566.

[118] Peierls, R., 1933, Z. Phys. 80, 763.

[119] Pichard, J. L. and G. Sarma, 1981, J. Phys. C 14, L127.

[120] Polyakov, D. G. and B. I. Shklovskii, 1993, Phys. Rev. Lett. 70, 3796.

[121] Pook, W. and J. Hajdu, 1987, Z. Phys. B 66, 427.

[122] Pook, W. and M. Janßen, 1991, Z. Phys. B 82, 295.

[123] Prange, R. E. and S. M. Girvin, Eds., 1987, The Quantum Hall Effect, Graduate Texts in Contemporary Physics, (Springer, Berlin).

[124] Prange, R. E. and R. Joynt, 1982, Phys. Rev. B 25, 2943.

[125] Pruisken, A. M. M., 1984, Nucl. Phys. B 235, 277. 
[126] Pruisken, A. M. M., 1987, chapter 5, p. 117-173, in (Prange and Girvin, 1987).

[127] Pruisken, A. M. M., 1988, Phys. Rev. Lett. 61, 1297.

[128] Pruisken, A. M. M. and L. Schäfer, 1981, Phys. Rev. Lett. 46, 490.

[129] Schäfer, L. and F. Wegner, 1980, Z. Phys. B 38, 113.

[130] Schweitzer, L., 1992, (unpublished).

[131] Schweitzer, L., B. Kramer, and A. MacKinnon, 1984, J. Phys. C 17, 4111.

[132] Shapiro, B., 1986, Phys. Rev. B 33, 8447.

[133] Shapiro, B., 1987, Philos. Mag. 56, 1031.

[134] Shimshoni, E. and S. L. Sondhi, 1994, Phys. Rev. B 49, 11484.

[135] Stauffer, D., 1979, Phys. Rep. 54, 2.

[136] Suzuki, M., 1977, Prog. Theor. Phys. 58, 1142.

[137] Thouless, D. J., 1974, Phys. Rep. 13, 93.

[138] Thouless, D. J., 1977, Phys. Rev. Lett. 39, 1167.

[139] Thouless, D. J., M. Kohmoto, M. P. Nightingale, and M. den Nijs, 1982, Phys. Rev. Lett. 49, 405.

[140] Trugman, S. A., 1983, Phys. Rev. B 27, 7539.

[141] Tsui, D. C., H. L. Störmer, and A. C. Gossard, 1982, Phys. Rev. Lett. 48, 1559.

[142] Tsukada, M., 1976, J. Phys. Soc. Jpn. 41, 1466.

[143] von Klitzing, K., G. Dorda, and M. Pepper, 1980, Phys. Rev. Lett. 45, 494.

[144] Wakabayashi, J., A. Fukaro, S. Kawaji, Y. Koike, and T. Fukose, 1990, Surf. Sci. 229, 60. 
[145] Wakabayashi, J., M. Yamane, and S. Kawaji, 1989, J. Phys. Soc. Jpn. 58, 1903.

[146] Wang, T., K. P. Clark, G. F. Spencer, A. M. Mack, and W. P. Kirk, 1994a, Phys. Rev. Lett. 72, 709.

[147] Wang, Z., D.-H. Lee, and X.-G. Wen, 1994b, Phys. Rev. Lett. 72, 2454.

[148] Wegner, F., 1976, Z. Phys. B 25, 327.

[149] Wegner, F., 1979, Z. Phys. B 35, 207.

[150] Wegner, F., 1980, Z. Phys. B 36, 209.

[151] Wegner, F., 1983, Z. Phys. B 51, 279.

[152] Wegner, F., 1989, Nucl. Phys. B 316, 663.

[153] Wei, H. P., L. W. Engel, and D. C. Tsui, 1994, Phys. Rev. B 50, 14609.

[154] Wei, H. P., S. W. Hwang, D. C. Tsui, and A. M. M. Pruisken, 1990, Surf. Sci. 229, 34.

[155] Wei, H. P., S. Y. Lin, D. C. Tsui, and A. M. M. Pruisken, 1992, Phys. Rev. B 45, 3926.

[156] Wei, H. P., D. C. Tsui, and A. M. M. Pruisken, 1985, Phys. Rev. B 33, 1488.

[157] Wei, H. P., D. C. Tsui, M. A. Paalanen, and A. M. M. Pruisken, 1987, in High Magnetic Fields in Semiconductor Physics, edited by G. Landwehr, Springer Series in Solid-State Sciences No. 71, (Springer, Berlin), p. 11.

[158] Wei, H. P., D. C. Tsui, M. A. Paalanen, and A. M. M. Pruisken, 1988, Phys. Rev. Lett. 61, 1294.

[159] Weidenmüller, H. A., 1987, Nucl. Phys. B 290 [FS20], 87.

[160] Weidenmüller, H. A. and M. R. Zirnbauer, 1988, Nucl. Phys. B 305[FS23], 339.

[161] Wilkinson, M., 1987, J. Phys. A: Math. Gen. 20, 1761. 
[162] Yamane, M., J. Wakabayashi, and S. Kawaji, 1989, J. Phys. Soc. Jpn. 58, 1899.

[163] Zak, J., 1964, Phys. Rev. 136, 776.

[164] Zirnbauer, M. R., 1994, Ann. Physik 3, 513. 


\section{FIGURES}

FIG. 1. The temperature dependence of the longitudinal and Hall conductivity [from (Kawaji and Wakabayashi, 1987)].

FIG. 2. Longitudinal conductivity $\sigma_{x x}$ vs. Hall conductivity $\sigma_{x y}$ in a Si-MOSFET for temperatures between $0.35 \mathrm{~K}$ and $1.5 \mathrm{~K}$ [from (Yamane et al., 1989)].

FIG. 3. Longitudinal conductivity $\sigma_{x x}$ vs. Hall conductivity $\sigma_{x y}$ in an InGaAs-InP heterostructure for temperatures between $50 \mathrm{mK}$ and $770 \mathrm{mK}$ [from (Wei et al., 1987)].

FIG. 4. Hall resistivity $\rho_{x y}$, longitudinal resistivity $\rho_{x x}$, and the derivative $d \rho_{x y} / d B$ of an InGaAs-InP heterostructure [from (Wei et al., 1988)].

FIG. 5. The temperature dependence of the maxima of $d \rho_{x y} / d B$ and the width $\Delta B$ of the $\rho_{x x}$ peaks from Fig. 4 . The slope of the straight lines gives $\left(d \rho_{x y} / d B\right)^{\max } \propto T^{-\kappa}$ and $\Delta B \propto T^{\kappa}$ with $\kappa=0.42 \pm 0.04$ [from (Wei et al., 1988)].

FIG. 6. Temperature dependence of the peak width $\Delta B$ for samples of different size [from (Koch et al., 1991a)].

FIG. 7. Real part of the dynamical conductivity $\operatorname{Re}\left(\sigma_{x x}\right)$ at different frequency and temperatures [from (Engel et al., 1993)].

FIG. 8. Regions where $V(\mathbf{r})<E$ are shaded. The eigenfunctions are localized within a distance $l_{c}$ of the edge of the regions. The arrow indicates the direction of guiding center motion [after (Trugman, 1983)].

FIG. 9. Equipotential lines of a smooth random potential. The thick lines correspond to the links in the network model. The circles enclose the saddle points [after (Chalker and Coddington, 1988)]. 
FIG. 10. The network model of Chalker and Coddington. Each node represents a saddle point and each link an equipotential line of the random potential [after (Chalker and Coddington, 1988)].

FIG. 11. The renormalization-group flow near a saddle-point fixed point $x_{1}=x_{2}=0$.

FIG. 12. $\beta$ function according to Abrahams et al. (1979). The slope of the dashed line is $1 / \nu$ [cf. Eq. (4.21)].

FIG. 13. Renormalization flow diagram of $\sigma_{x x}$ vs. $\sigma_{x y}$ [after (Khmel'nitskii, 1983)].

FIG. 14. Normalized localization length $\lambda_{M} / M$ in the lowest Landau level for $\delta$-correlated potential as a function of system width $M$ (in units of $\sqrt{2 \pi} l_{c}$ ) for energies $0.01(*), 0.05(\nabla), 0.07$ $(\triangle), 0.1(\square), 0.18(\diamond), 0.30(\star), 0.5(\circ)$, and $1.0(\bullet)$ (in units of $\Gamma$ ) [after (Huckestein and Kramer, 1990)].

FIG. 15. The scaling function $\lambda_{M}(E) / M=\Lambda(M / \xi(E))$ [from (Huckestein, 1992)].

FIG. 16. The localization length $\xi(E)$ as a function of energy normalized by the band width $\Gamma_{\sigma}$ for $n=0, \sigma=0(\nabla), n=0, \sigma=l_{c}(\triangle)$, and $n=1, \sigma=l_{c}(\square)$ [after (Huckestein, 1992)].

FIG. 17. Normalized localization length $\lambda_{M} / M$ in the lowest Landau level for correlated potential $\left(\sigma=l_{c}\right)$ as a function of system width $M$ (in units of $\left.\sqrt{2 \pi} l_{c}\right)$ for energies $0(*), 0.03(\times)$, $0.05(\nabla), 0.07(\triangle), 0.1(\square), 0.14(\star), 0.18(\diamond), 0.3(\circ)$, and $0.5(\bullet)$ (in units of $\Gamma$ ) [after (Huckestein et al., 1992)].

FIG. 18. Normalized localization length $\lambda_{M} / M$ in the second $(n=1)$ Landau level for correlated potential $\left(\sigma=l_{c}\right)$ as a function of system width $M$ (in units of $\left.\sqrt{2 \pi} l_{c}\right)$ for energies $0.01(*)$, $0.03(\nabla), 0.05(\triangle), 0.07(\square), 0.1(\diamond), 0.18(\star), 0.3(\circ)$, and $0.5(\bullet)$ (in units of $\Gamma$ ) [from (Huckestein, 1992)]. 
FIG. 19. Normalized localization length $\lambda_{M} / M$ in the second $(n=1)$ Landau level for uncorrelated potential $(\sigma=0)$ as a function of system width $M$ (in units of $\sqrt{2 \pi} l_{c}$ ) for energies $0.01(*)$, $0.1(\nabla), 0.18(\triangle), 0.3(\square), 0.5(\diamond), 0.65(\star), 0.8(\circ)$, and $1.0(\bullet)$ (in units of $\Gamma$ ) [from (Huckestein, 1992)].

FIG. 20. The normalized localization length $\lambda_{M} / M$ at the band center of the second Landau level as function of $\beta^{2}=\left(\sigma^{2}+l_{c}^{2}\right) / l_{c}^{2}$ for system width $M=16(\circ), M=32(\bullet), M=64(\diamond)$, and $M=128(*)$ [after (Huckestein, 1992)].

FIG. 21. Scaling towards the fixed point value $\Lambda_{c}=1.14$ as a function of $M / \xi_{\text {irr }}$ for the data shown in Fig. 20 [from (Huckestein, 1994)].

FIG. 22. The irrelevant length scale $\xi_{\text {irr }}$ (in units of $\sqrt{2 \pi} l_{c}$ ) as a function of $\beta^{2}$ [from (Huckestein, 1994)].

FIG. 23. Deviations of the scaling function $\Lambda$ from its asymptotic value $\Lambda_{c}$ [from (Huckestein, 1994)].

FIG. 24. Phase diagram of the lowest Landau level in the presence of a periodic potential with $\alpha=3 / 5$ unit cells per flux quantum. Indicated are the positions of the critical energies and the Hall conductivity in the different phases. The bars at zero $\Gamma / E_{0}$ show the subbands in the absence of disorder.

FIG. 25. The local density $|\psi(\mathbf{r})|^{2}$ of an eigenstate at the center of the lowest Landau level of a lattice system with $150 \times 150$ sites [from (Huckestein and Schweitzer, 1992a)].

FIG. 26. The $f(\alpha)$ spectrum of the local density of the eigenstate shown in Fig. 25. Crosses show the errors in $f(\alpha(q))$ and $\alpha(q)$ for $q=-2,-1,1$, and 2 (from right to left) [from (Huckestein et al., 1992)]. 
FIG. 27. The $f(\alpha)$ spectrum of the local equilibrium current density of the eigenstate shown in Fig. 25. Crosses show the errors in $f(\alpha(q))$ and $\alpha(q)$ for $q=-2,-1,1$, and 2 (from right to left) [from (Huckestein and Schweitzer, 1992a)].

FIG. 28. The energy dependence of $\alpha_{0}(E)$ near the center of the lowest Landau band. The straight line is a guide to the eye [from (Huckestein and Schweitzer, 1992b)]. 


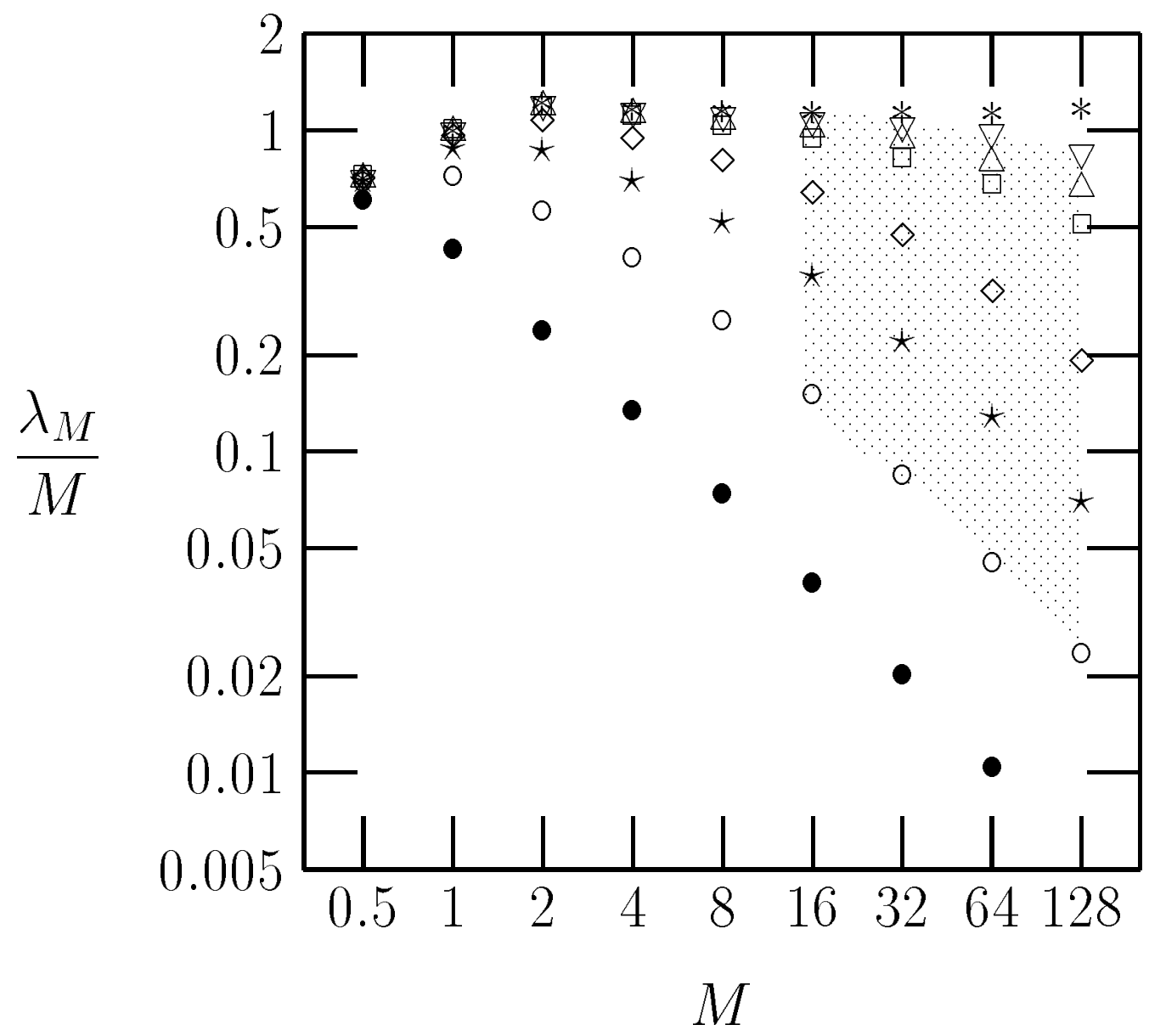




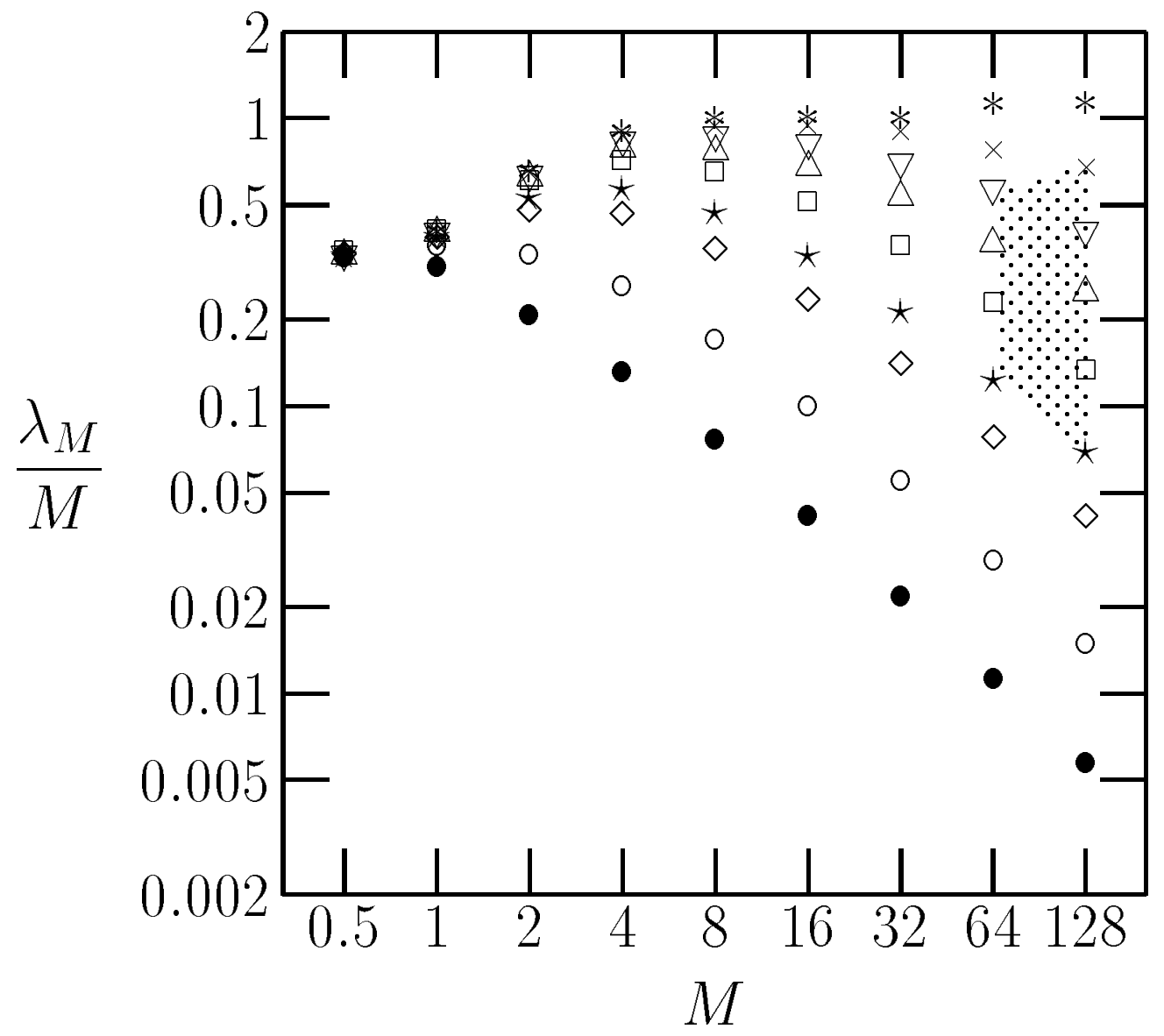




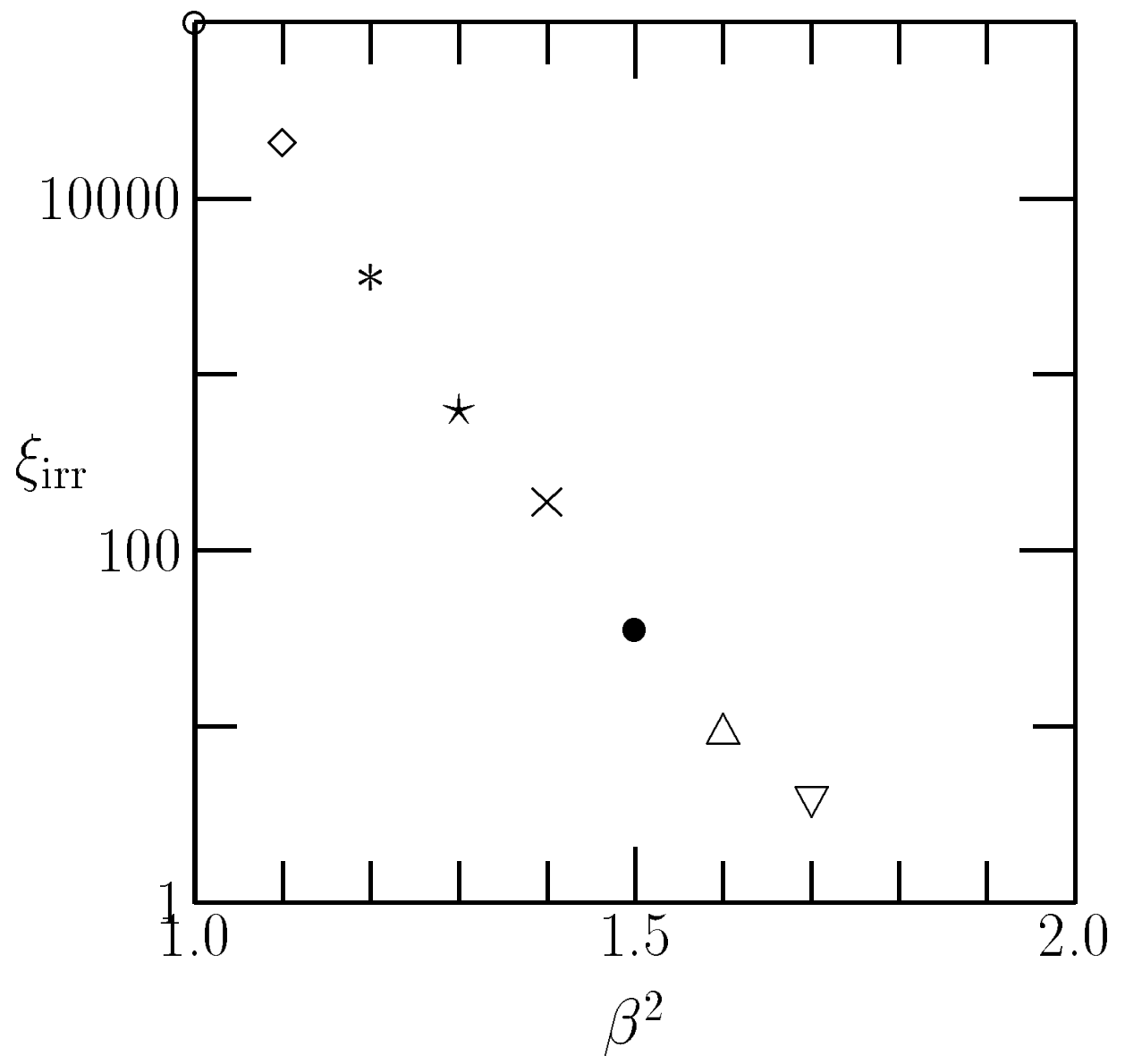




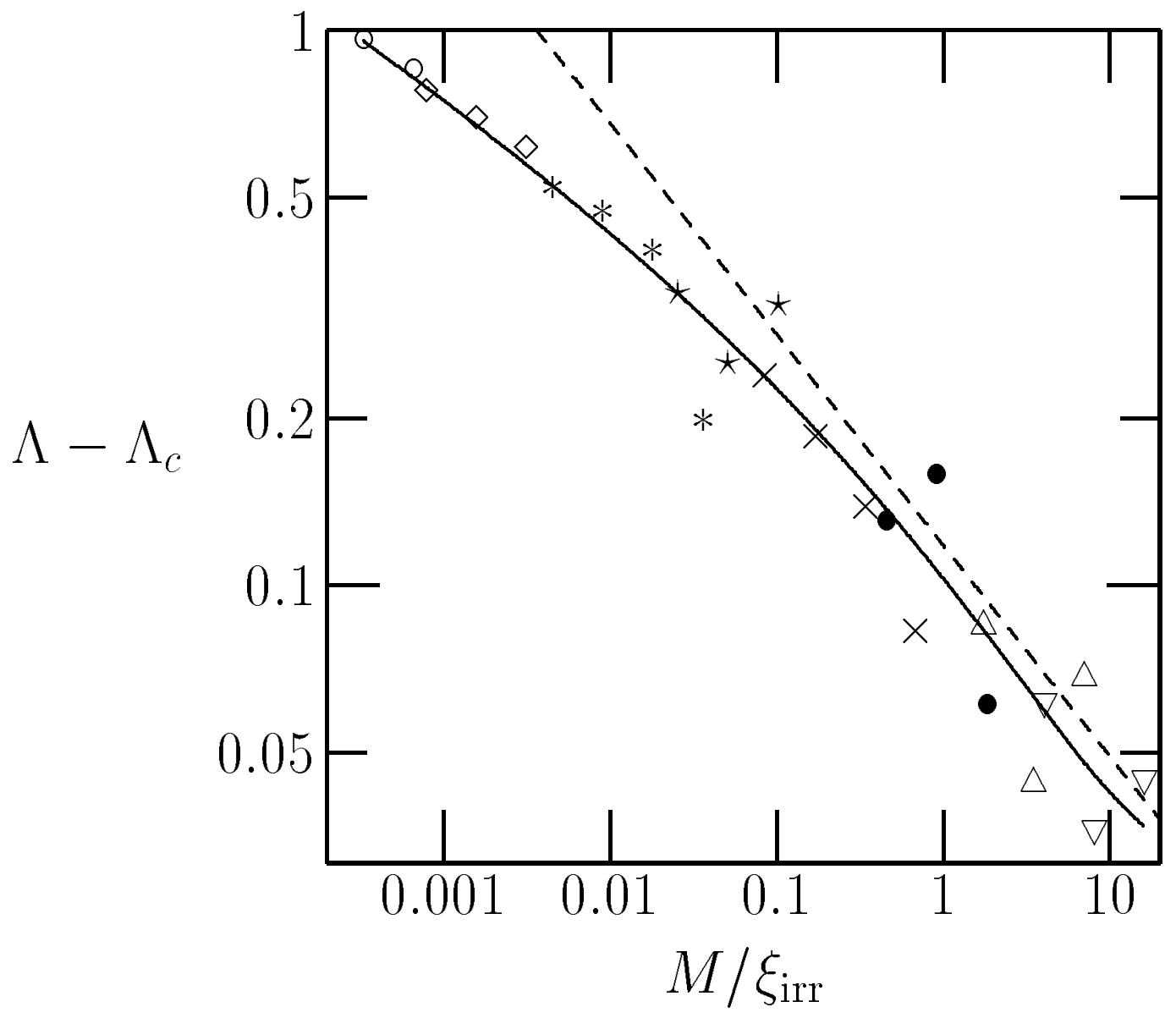




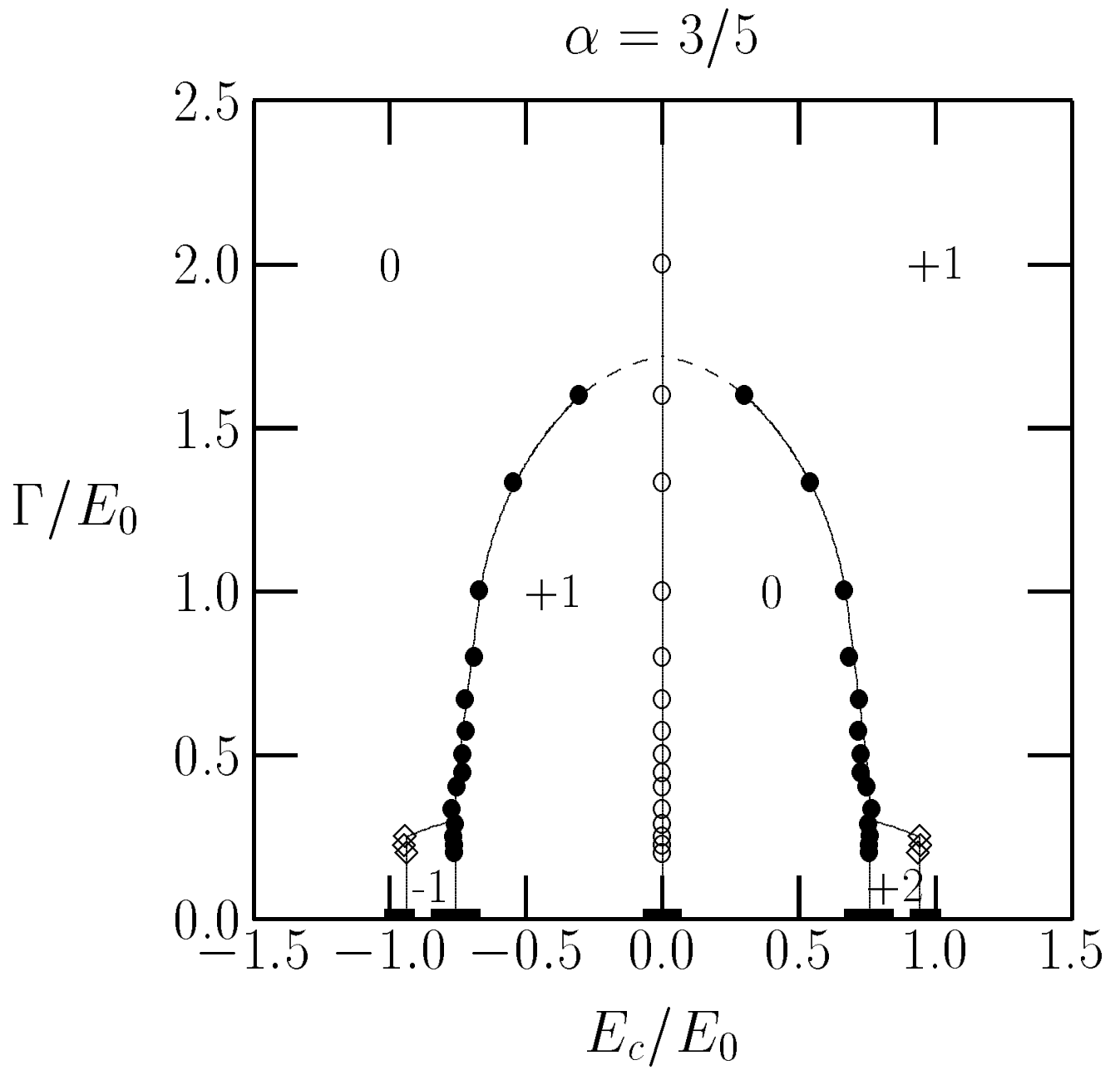




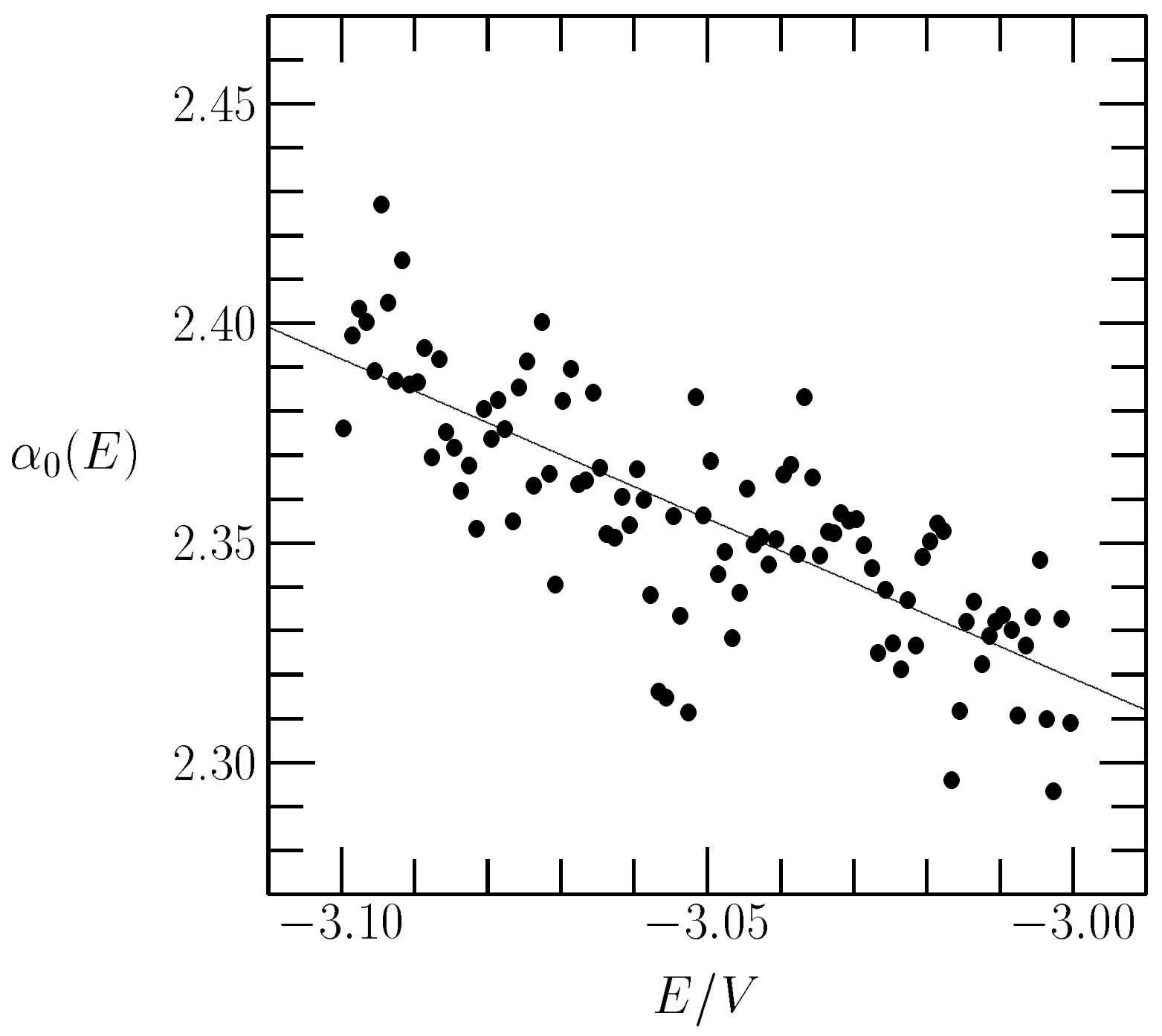




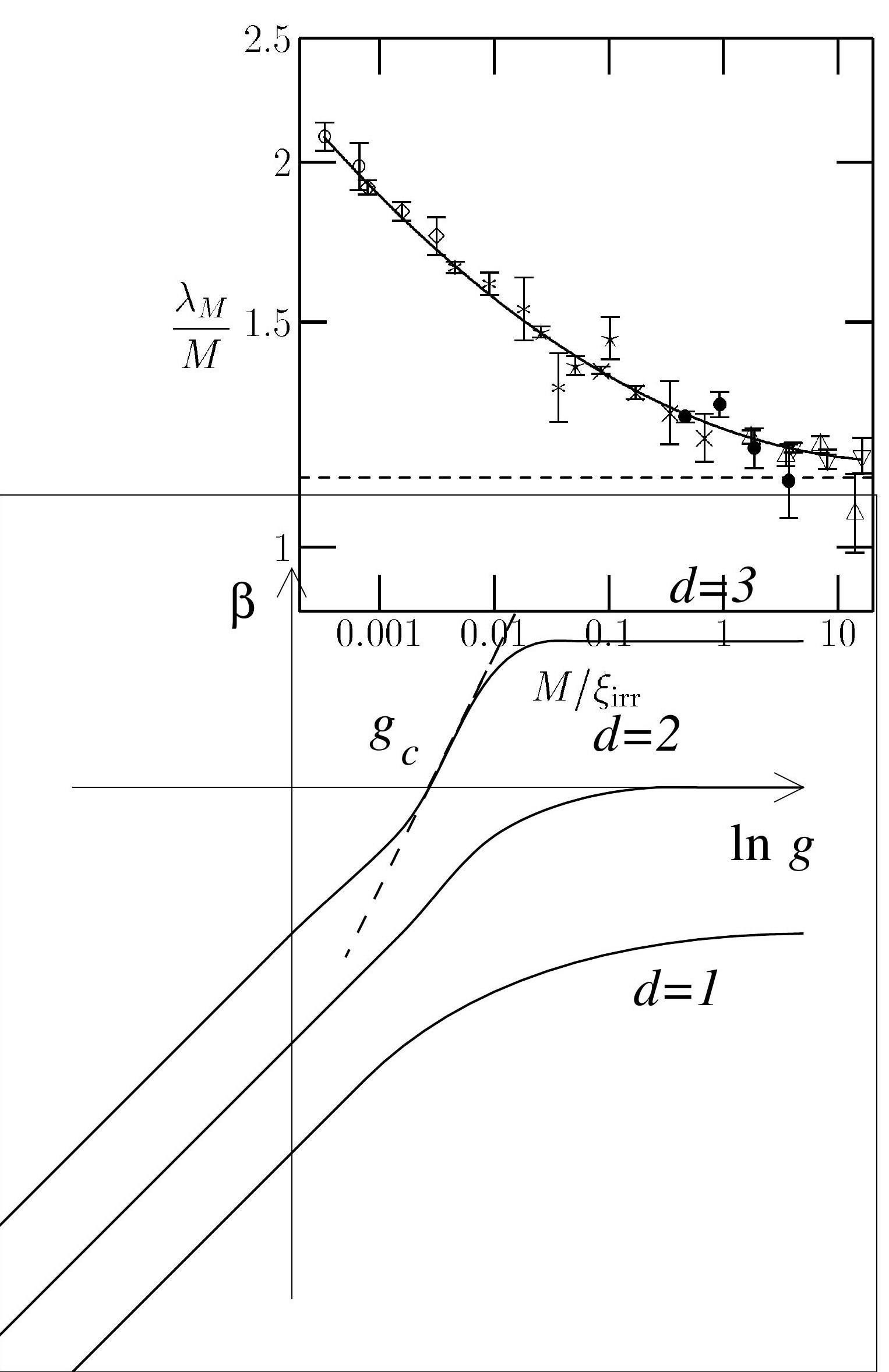




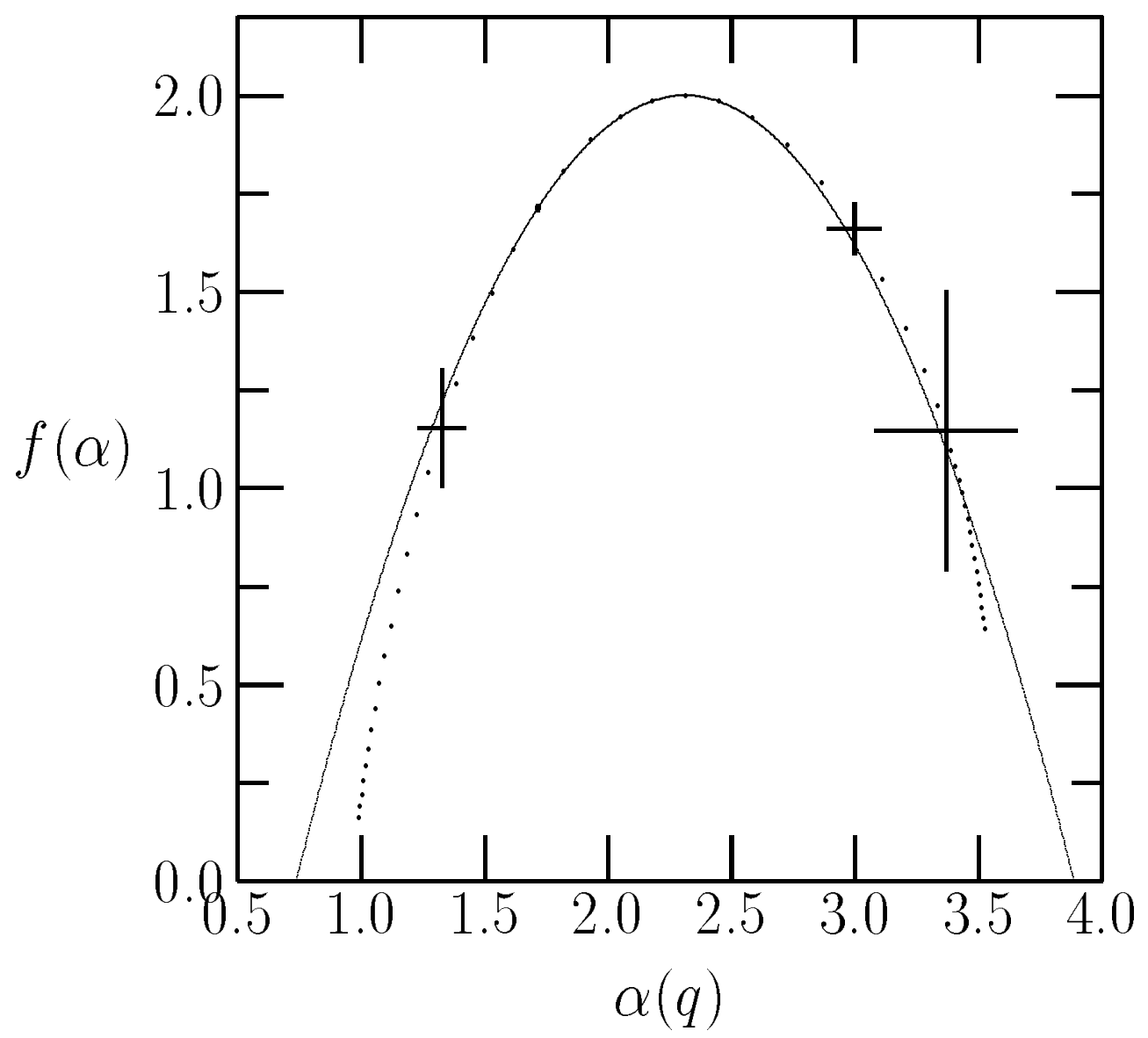




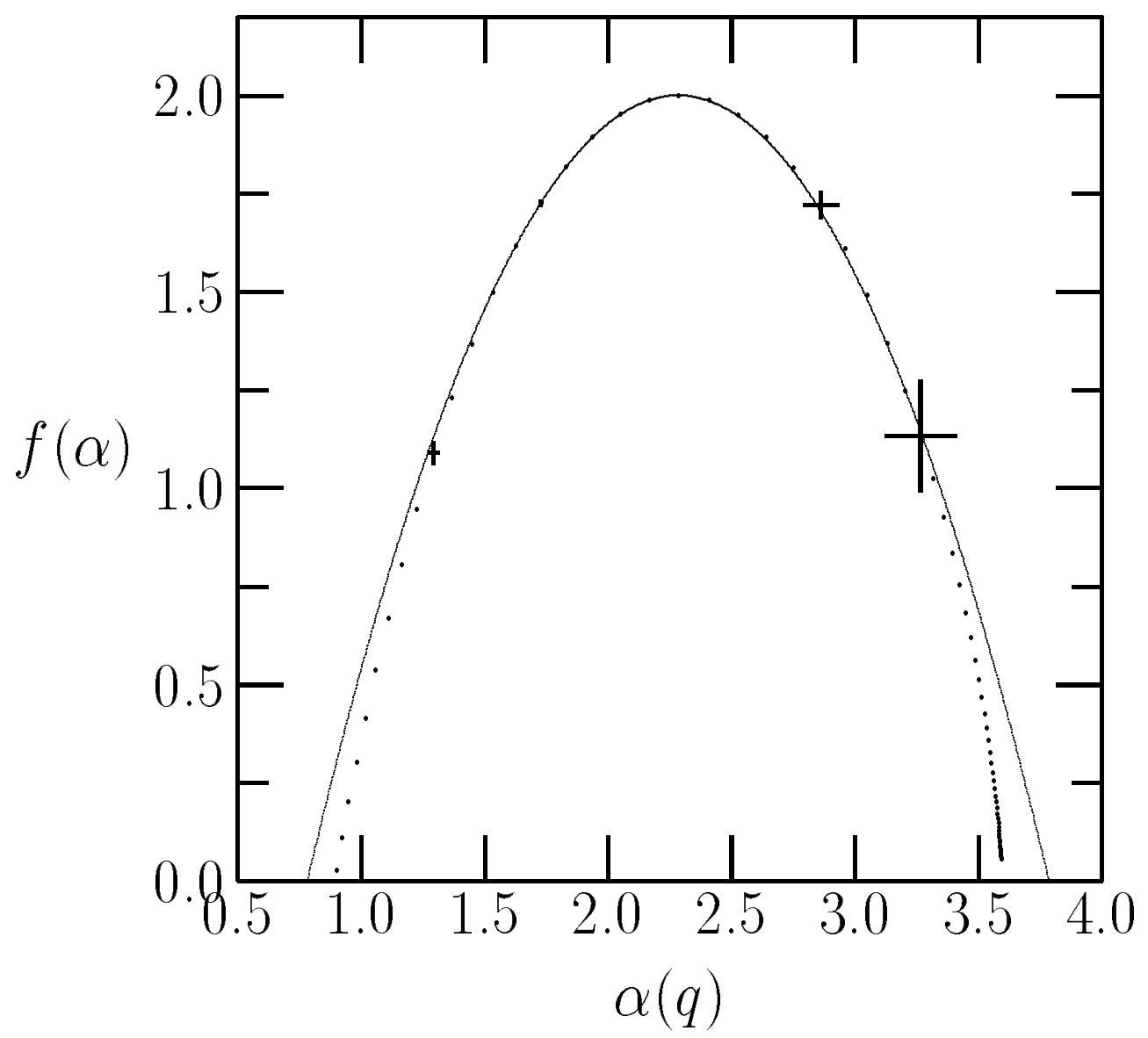




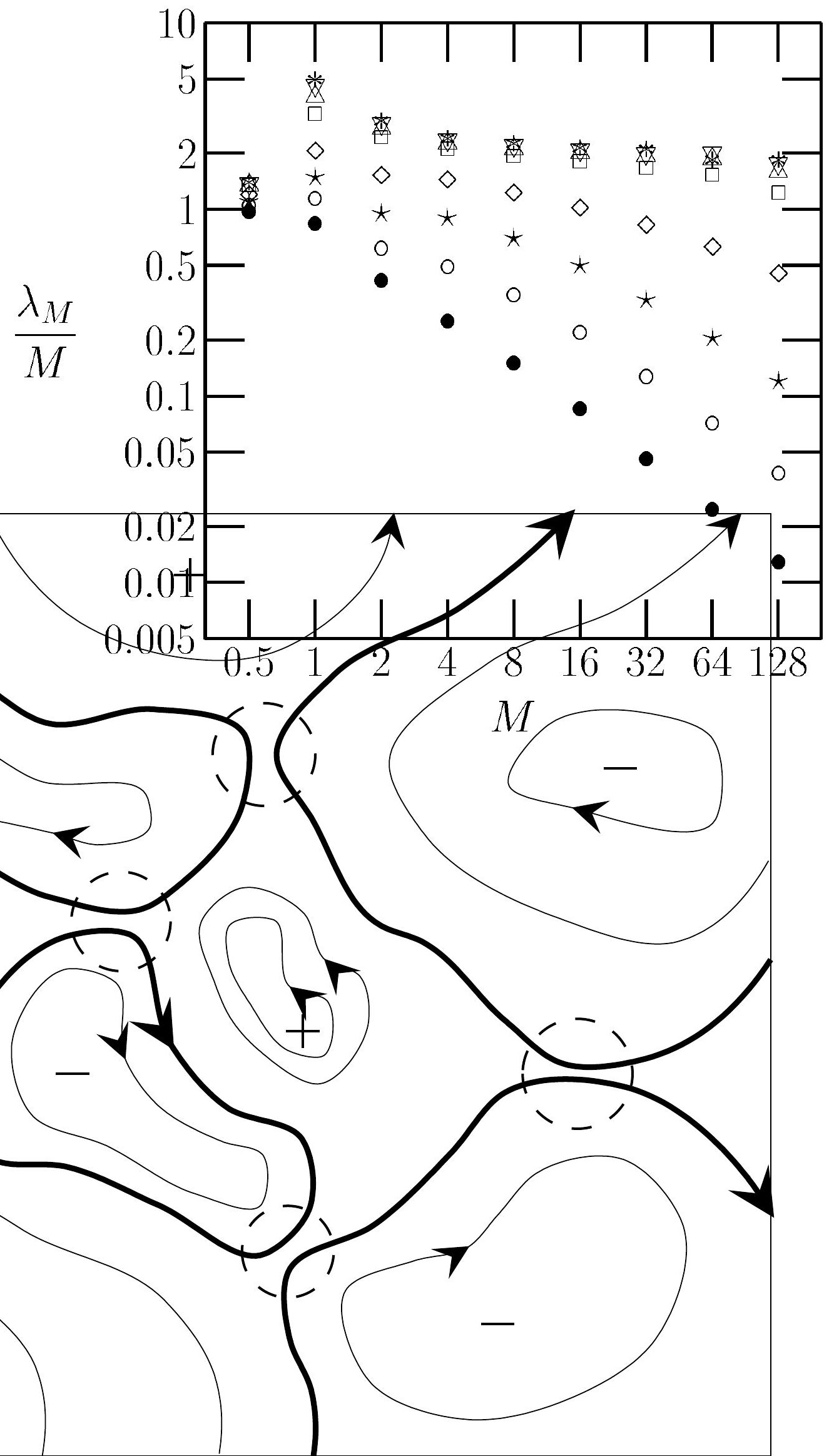




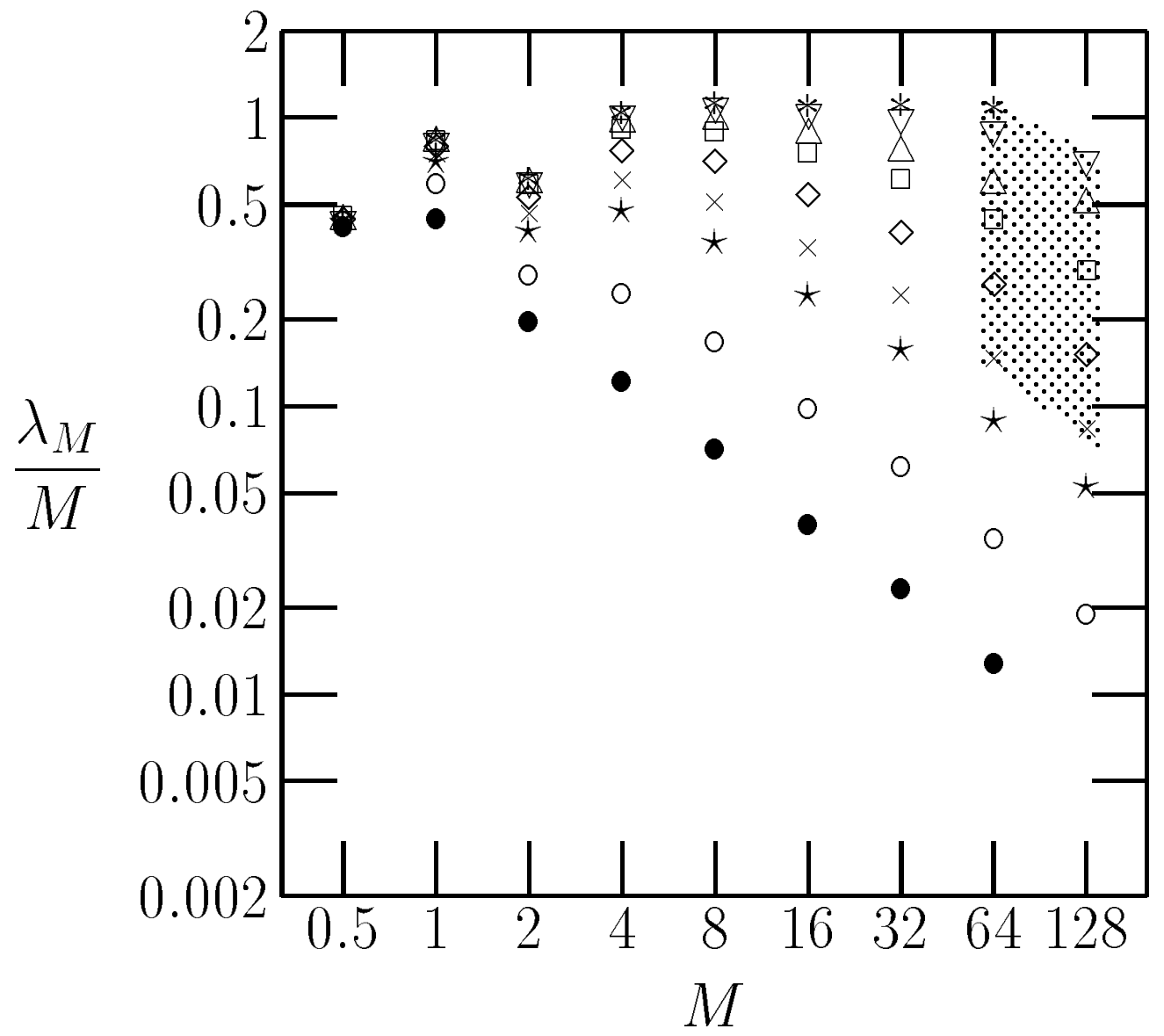




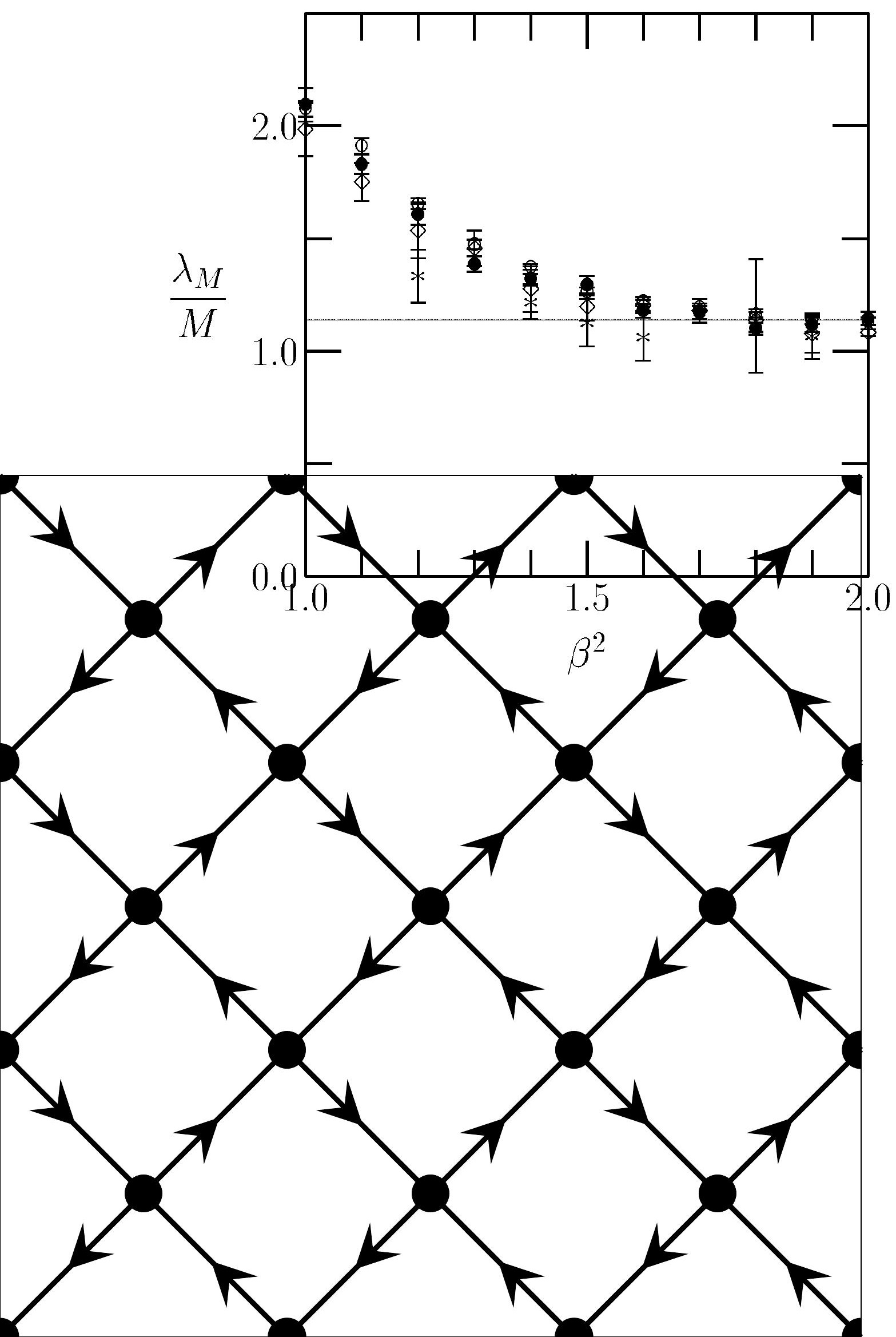




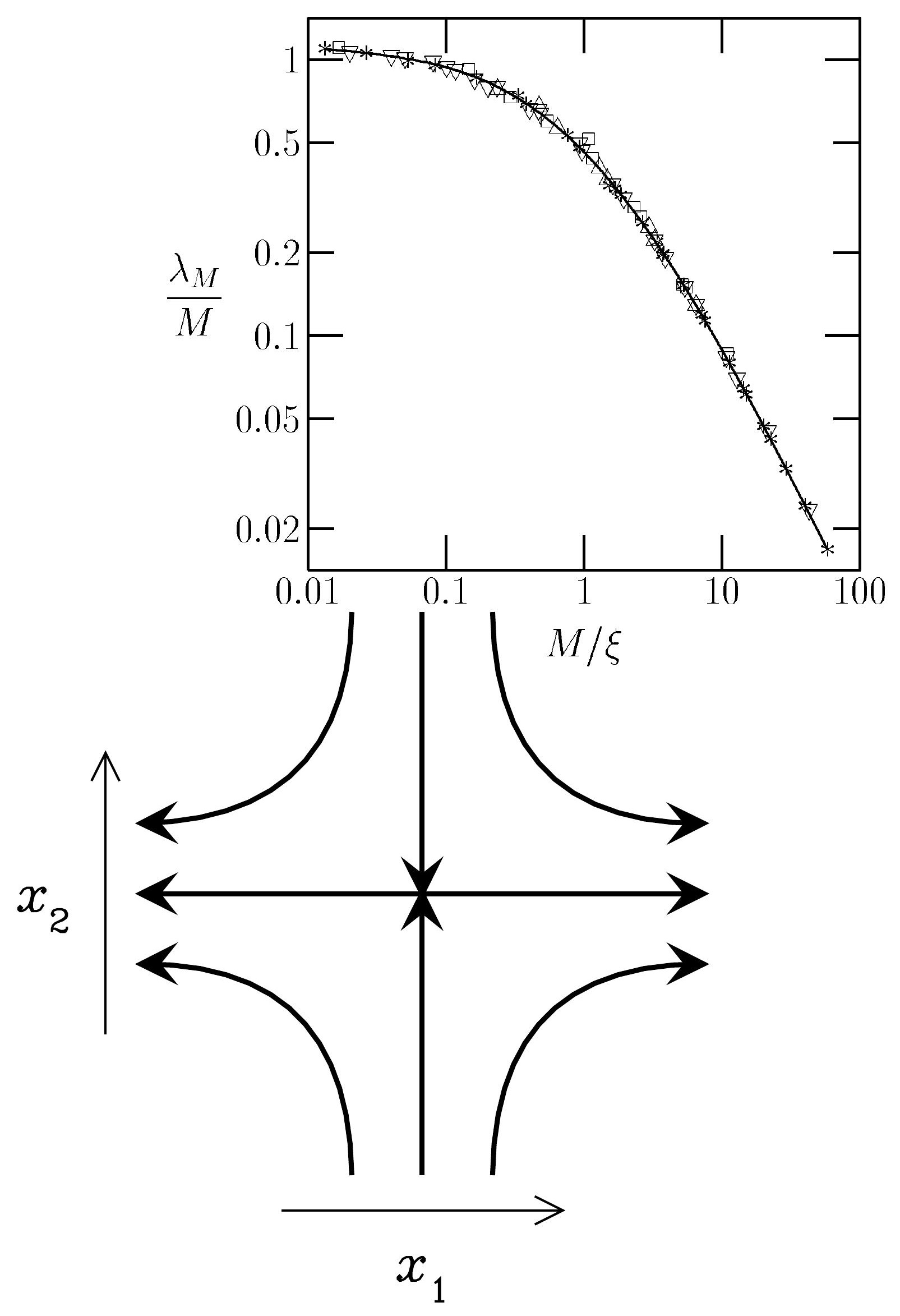




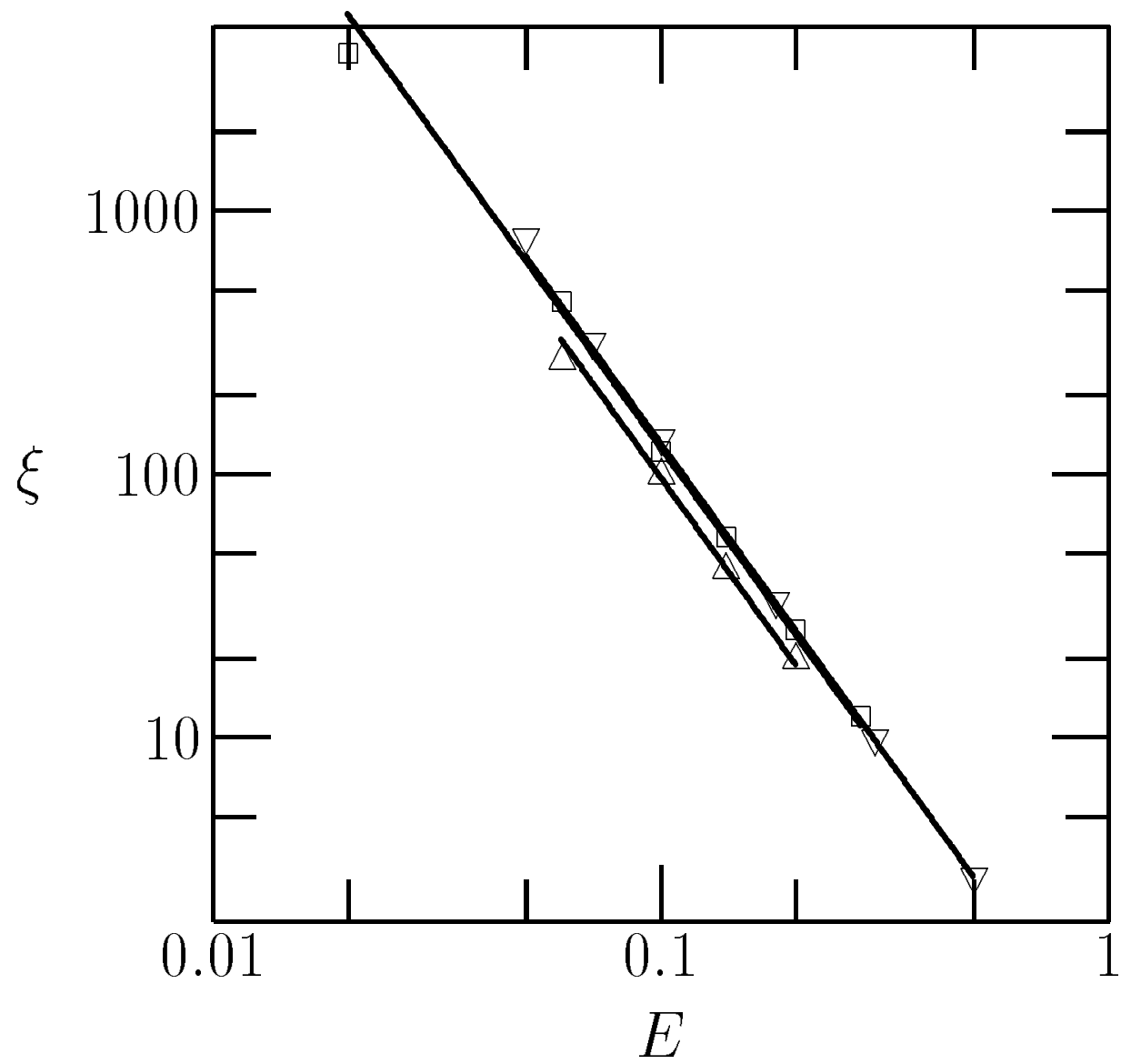

